\title{
AUBRY SETS AND THE DIFFERENTIABILITY OF THE MINIMAL AVERAGE ACTION IN CODIMENSION ONE
}

\author{
UGO BESSI ${ }^{1}$
}

\begin{abstract}
Let $\mathcal{L}(x, u, \nabla u)$ be a Lagrangian periodic of period 1 in $x_{1}, \ldots, x_{n}, u$. We shall study the non self intersecting functions $u: \mathbf{R}^{n} \rightarrow \mathbf{R}$ minimizing $\mathcal{L}$; non self intersecting means that, if $u\left(x_{0}+k\right)+j=u\left(x_{0}\right)$ for some $x_{0} \in \mathbf{R}^{n}$ and $(k, j) \in \mathbf{Z}^{n} \times \mathbf{Z}$, then $u(x)=u(x+k)+j \forall x$. Moser has shown that each of these functions is at finite distance from a plane $u=\rho \cdot x$ and thus has an average slope $\rho$; moreover, Senn has proven that it is possible to define the average action of $u$, which is usually called $\beta(\rho)$ since it only depends on the slope of $u$. Aubry and Senn have noticed a connection between $\beta(\rho)$ and the theory of crystals in $\mathbf{R}^{n+1}$, interpreting $\beta(\rho)$ as the energy per area of a crystal face normal to $(-\rho, 1)$. The polar of $\beta$ is usually called $-\alpha$; Senn has shown that $\alpha$ is $C^{1}$ and that the dimension of the flat of $\alpha$ which contains $c$ depends only on the "rational space" of $\alpha^{\prime}(c)$. We prove a similar result for the faces (or the faces of the faces, etc.) of the flats of $\alpha$ : they are $C^{1}$ and their dimension depends only on the rational space of their normals.
\end{abstract}

Mathematics Subject Classification. 35J20, 35J60

Received November 3, 2006. Revised July 23, 2007.

Published online March 6, 2008.

\section{INTRODUCTION}

We begin recalling some results of [17]. Let $\mathcal{L}\left(x_{1}, \ldots, x_{n}, u, p_{1}, \ldots, p_{n}\right)$ be a Lagrangian such that

1) $\mathcal{L} \in C^{l, \gamma}\left(\mathbf{R}^{2 n+1}\right), l \geq 2, \gamma>0$.

2) $\mathcal{L}$ has period 1 in $x_{1}, \ldots, x_{n}, u$.

3) There is $\delta>0$ such that

$$
\delta I \leq \frac{\partial^{2} \mathcal{L}}{\partial p_{i} \partial p_{j}} \leq \frac{1}{\delta} I
$$

where $I$ denotes the identity matrix on $\mathbf{R}^{n}$.

4) There is $C>0$ such that

$$
\begin{gathered}
\left|\frac{\partial^{2} \mathcal{L}}{\partial p \partial x}\right|+\left|\frac{\partial^{2} \mathcal{L}}{\partial p \partial u}\right| \leq C(1+|p|) \\
\left|\frac{\partial^{2} \mathcal{L}}{\partial x \partial x}\right|+\left|\frac{\partial^{2} \mathcal{L}}{\partial u \partial x}\right|+\left|\frac{\partial^{2} \mathcal{L}}{\partial u \partial u}\right| \leq C\left(1+|p|^{2}\right) .
\end{gathered}
$$

Keywords and phrases. Aubry-Mather theory for elliptic problems, corners of the mean average action.

1 Dipartimento di Matematica, Università Roma Tre, Largo S. Leonardo Murialdo, 00146 Roma, Italy.

bessi@matrm3.mat.uniroma3.it 
We say that $u \in W_{l o c}^{1,2}\left(\mathbf{R}^{n}\right)$ is a minimizer for $\mathcal{L}$ if

$$
\int_{\mathbf{R}^{n}}[\mathcal{L}(x, u+\phi, \nabla(u+\phi))-\mathcal{L}(x, u, \nabla u)] \mathrm{d} x \geq 0 \quad \forall \phi \in C_{0}^{\infty}\left(\mathbf{R}^{n}\right) .
$$

Since $\mathcal{L}$ is periodic, if $u$ is a minimizer and $(k, j) \in \mathbf{Z}^{n} \times \mathbf{Z}$, then $u(x+k)+j$ is a minimizer too; we say that $u$ is non self intersecting if

$$
\begin{gathered}
\forall(k, j) \in \mathbf{Z}^{n} \times \mathbf{Z}, \quad \text { either } \quad u(x+k)+j>u(x) \forall x \\
\text { or } u(x+k)+j<u(x) \forall x \quad \text { or } \quad u(x+k)+j=u(x) \forall x .
\end{gathered}
$$

It is proven in [17] that, if $u$ satisfies (1) and (2), then $u$ is at finite distance from a plane; more precisely, there is $\rho \in \mathbf{R}^{n}$ such that, for $1 \leq k \leq l$,

$$
\|u(x)-u(0)-\rho \cdot x\|_{C^{k, \gamma}\left(\mathbf{R}^{n}\right)} \leq M_{k}(\|\rho\|)
$$

where $\rho \cdot x$ denotes the scalar product and $\gamma$ is the same as in 1$)$. The vector $\rho$, which is clearly unique, is called the slope, or rotation vector, of $u$. Since $l \geq 2, u \in C^{2}\left(\mathbf{R}^{n}\right)$ and $u$ is a classical solution of the Euler-Lagrange equation of (1):

$$
\operatorname{div} \frac{\partial \mathcal{L}(x, u, \nabla u)}{\partial p}=\frac{\partial \mathcal{L}(x, u, \nabla u)}{\partial u}
$$

Let $M_{\rho}$ denote the set of all minimal, non self intersecting $u$ of slope $\rho$; in [17] it is proven that $M_{\rho}$ is never empty.

In [20] it is proven that, if $u \in M_{\rho}$, then the following limit exists:

$$
\lim _{R \rightarrow+\infty} \frac{1}{|B(0, R)|} \int_{B(0, R)} \mathcal{L}(x, u, \nabla u) \mathrm{d} x
$$

Moreover, the limit above does not depend on the particular $u \in M_{\rho}$ we choose, and we can call it $\beta(\rho)$. The function $\beta$ is strictly convex and superlinear, thus its polar, usually called $-\alpha$, is of class $C^{1}$. We shall study the differentiability of $\beta$. This problem is motivated by an observation of Gibbs', recalled in [2] and [22], which says that $\frac{1}{\sqrt{1+|\rho|^{2}}} \beta(\rho)$ can be interpreted as the energy per unit of area of the face of a $(n+1)$-dimensional crystal which is orthogonal to $(-\rho, 1)$. This energy is called a Wulff functional by crystalline people (see for instance [23]); we want to study what kind of corners are possible for Wulff functionals which arise from a microscopic theory like that of Gibbs'.

Following [13], instead of studying the corners of $\beta$, we shall study the flats of its polar which is traditionally [15] called $-\alpha$. We recall that, if $\rho \in \mathbf{R}^{n}$, then we can always find a unimodular matrix $A, 0 \leq s \leq n$, $\left(\rho_{1}, \ldots, \rho_{s}\right) \in \mathbf{Q}^{s}$ and $\left(\rho_{s+1}, \ldots, \rho_{n}\right)$ rationally independent, such that

$$
A \rho=\left(\rho_{1}, \ldots, \rho_{s}, \rho_{s+1}, \ldots, \rho_{n}\right) .
$$

We set $A^{-1}\left(\mathbf{R}^{s} \times\{0\}\right)=\operatorname{rat}(\rho)$, the rational space of $\rho$. We recall a theorem of [21]: let $-\alpha^{\prime}(c)=\rho$, and let $D_{\rho}$ be the flat of $\alpha$ containing $c$. Then either $D_{\rho}$ is reduced to a point, or it generates $\operatorname{rat}(\rho)$. The first case happens iff $M_{\rho}$ is an ordered set.

The theorem we prove in this paper, Theorem 2.1 below, deals with the faces and subfaces of $D_{\rho}$; we state it now in a rather vague form because we haven't defined yet many of the objects involved. Let us suppose that $D_{\rho}$ does not reduce to a point; we restrict ourselves to the smallest affine subspace containing $D_{\rho}$ and we denote by $\partial D_{\rho}$ the boundary of $D_{\rho}$ relative to this space. We shall show that every point of $\partial D_{\rho}$ admits a unique normal; in particular, since $D_{\rho}$ is convex, $\partial D_{\rho}$ is of class $C^{1}$. Moreover, if $c \in \partial D_{\rho}$ and $v_{1}$ is the normal to $\partial D_{\rho}$ at $c$, then the dimension of the face of $D_{\rho}$ containing $c$ is either zero, or an integer depending only on $\rho$ and $v_{1}$; it is zero iff a certain subset $M_{\left(\rho, v_{1}\right)}$ of $M_{\rho}$ is ordered. Similar results on dimension and $C^{1}$ regularity hold for the faces of the faces of $D_{\rho}$, down to the 0 -dimensional faces. 
We conclude with a brief history of this problem. Aubry in [2] was the first to study the function $\beta$ when $n=1$; he conjectured that $\beta$ is differentiable at $\rho$ if $\rho$ is an irrational number. This conjecture has been proven in [14] and [5]; the theorem has been extended in [21] to all $n$. The paper [3] considers the corners of the stable norm, i.e. the same problem as [21], but for the area functional. The papers $[7,12,13,18]$ consider the case of 1-dimensional currents on compact manifolds. Our method is a linear combination of [13] and [21].

\section{Preliminaries}

In the following, it will be convenient to consider the current induced by $u \in M_{\rho}$; in this section, following [6,8], we show how the mean action of $u$ coincides with the action of the current it induces.

Let $T$ be a $n$-current of finite mass on $\mathbf{T}^{n+1}=\frac{\mathbf{R}^{n+1}}{\mathbf{Z}^{n+1}}$; we suppose that $T$ is closed, i.e. that $T(\eta)=0$ whenever $\eta$ is exact. In particular, if $\eta$ is a closed form, $T(\eta)$ depends only on its cohomology class $[\eta]$, and we can define a linear mapping

$$
\begin{gathered}
\rho_{T}: H^{n}\left(\mathbf{T}^{n+1}\right) \rightarrow \mathbf{R} \\
\rho_{T}:[\eta] \rightarrow T(\eta) .
\end{gathered}
$$

Since $H_{n}\left(\mathbf{T}^{n+1}\right)$ is the dual of $H^{n}\left(\mathbf{T}^{n+1}\right)$, we can identify the rotation number $\rho_{T}$ with an element of $H_{n}\left(\mathbf{T}^{n+1}\right)$.

On $H^{n}\left(\mathbf{T}^{n+1}\right)$ we have the basis $\widehat{\mathrm{d} x_{i}}=(-1)^{n-i} \mathrm{~d} x_{1} \wedge \ldots \wedge \mathrm{d} x_{i-1} \wedge \mathrm{d} x_{i+1} \wedge \ldots \wedge \mathrm{d} x_{n+1}$ for $i \in(1, \ldots, n)$, and $\widehat{\mathrm{d} x_{n+1}}=\mathrm{d} x_{1} \wedge \ldots \wedge \mathrm{d} x_{n}$; on $H_{n}\left(\mathbf{T}^{n+1}\right)$ we have the basis $\left\{e_{i}\right\}_{i=1}^{n+1}$ dual to $\left\{\widehat{\mathrm{d} x_{i}}\right\}_{i=1}^{n+1}$. For $u \in M_{\rho}$, we define the current $T_{u}$ by

$$
T_{u}(\eta)=\lim _{R \rightarrow \infty} \frac{1}{|B(0, R)|} \int_{B(0, R)} \eta(x, u(x)) \cdot \nabla u(x) \mathrm{d} x
$$

where we have denoted by $\eta(x, u(x)) \cdot \nabla u(x)$ the $n$-form $\eta$ applied the $n$-vector

$$
\left(\begin{array}{cccc}
1 & 0 & \ldots & 0 \\
0 & 1 & \ldots & 0 \\
\ldots & \ldots & \ldots & \ldots \\
0 & 0 & \ldots & 1 \\
\partial_{1} u & \partial_{2} u & \ldots & \partial_{n} u
\end{array}\right)
$$

To show that the limit in (1.1) exists, we borrow some facts from the beginning of Section 2. If $\rho \in \mathbf{Q}^{n}$, then $M_{\rho}$ contains periodic elements $u$, which means that $u(x+k)+j=u(x)$ if $(k, j) \in\left(\mathbf{Z}^{n} \times \mathbf{Z}\right) \cap(\rho, 1)^{\perp}$. For these elements, the limit of (1.1) exists trivially. If $u \in M_{\rho}$ but $u$ is not periodic, then there are $u_{1}, u_{2} \in M_{\rho}$ periodic and $v \in \mathbf{R}^{n}$ such that

$$
\lim _{t \rightarrow-\infty}\left\|u-u_{1}\right\|_{C^{1}(\langle x, v\rangle<t)}=0=\lim _{t \rightarrow+\infty}\left\|u-u_{2}\right\|_{C^{1}(t<\langle x, v\rangle)} .
$$

Thus $u$ is asymptotic to $u_{1}$ and $u_{2}$, for which the limit in (1.1) exists; for $M \geq 0$ we write

$$
\begin{aligned}
& \frac{1}{|B(0, R)|} \int_{B(0, R)} \eta(x, u(x)) \cdot \nabla u(x) \mathrm{d} x=\frac{1}{|B(0, R)|} \int_{B(0, R) \cap\{\langle x, v\rangle \leq-M\}} \eta(x, u(x)) \cdot \nabla u(x) \mathrm{d} x \\
& \quad+\frac{1}{|B(0, R)|} \int_{B(0, R) \cap\{\langle x, v\rangle \geq M\}} \eta(x, u(x)) \cdot \nabla u(x) \mathrm{d} x+\frac{1}{|B(0, R)|} \int_{B(0, R) \cap\{-M \leq\langle x, v\rangle \leq M\}} \eta(x, u(x)) \cdot \nabla u(x) \mathrm{d} x .
\end{aligned}
$$

Since $u$ is asymptotic to $u_{1}$ and $u_{2}$ we can fix $M$ large enough to have

$$
\begin{aligned}
& \limsup _{R \rightarrow \infty} \frac{1}{|B(0, R)|} \int_{B(0, R) \cap\{\langle x, v\rangle \leq-M\}}\left|\eta(x, u(x)) \cdot \nabla u(x)-\eta\left(x, u_{1}(x)\right) \cdot \nabla u_{1}(x)\right| \mathrm{d} x \leq \epsilon \\
& \limsup _{R \rightarrow \infty} \frac{1}{|B(0, R)|} \int_{B(0, R) \cap\{\langle x, v\rangle \geq M\}}\left|\eta(x, u(x)) \cdot \nabla u(x)-\eta\left(x, u_{2}(x)\right) \cdot \nabla u_{2}(x)\right| \mathrm{d} x \leq \epsilon .
\end{aligned}
$$


Since $|\eta(x, u(x)) \cdot \nabla u(x)|$ is bounded by (3), we have that, for $M$ fixed as above and $R$ large enough,

$$
\frac{1}{|B(0, R)|} \int_{B(0, R) \cap\{-M \leq\langle x, v\rangle \leq M\}}|\eta(x, u(x)) \cdot \nabla u(x)| \mathrm{d} x \leq \epsilon .
$$

Since the limit in (1.1) exists for $u_{1}$ and $u_{2}$, we get from the last four formulas that it exists also for $u$.

If $\rho \notin \mathbf{Q}^{n}$, then the recurrent elements of $M_{\rho}$ can be parameterized by $y \in \mathbf{R}$ in the following way: $u(x, y)=$ $U(x, \rho \cdot x+y)$, where $U\left(x, x_{n+1}\right)-x_{n+1}$ is bounded and periodic of period 1 in $x_{1}, \ldots, x_{n}, x_{n+1}$. In particular, $u(x+k, y)=u(x, y+\rho \cdot k)$. Now we note that

$$
\begin{gathered}
\lim _{R \rightarrow \infty} \frac{1}{|B(0, R)|} \int_{B(0, R)} \eta(x, u(x, y)) \cdot \nabla_{x} u(x, y) \mathrm{d} x= \\
\lim _{R \rightarrow \infty} \frac{1}{\#\left\{k \in \mathbf{Z}^{n}:|k| \leq R\right\}} \sum_{|k| \leq R} \int_{[0,1]^{n}} \eta(x+k, u(x+k, y)) \nabla_{x} u(x+k, y) \mathrm{d} x \\
\quad=\lim _{R \rightarrow \infty} \frac{1}{\#\left\{k \in \mathbf{Z}^{n}:|k| \leq R\right\}} \sum_{|k| \leq R} \int_{[0,1]^{n}} \eta(x, u(x, y+\rho \cdot k)) \nabla_{x} u(x, y+\rho \cdot k) \mathrm{d} x
\end{gathered}
$$

The limit of the last quantity exists by the ergodic theorem for $\mathbf{Z}^{n}$ actions of [25]; we apply it to the $\mathbf{Z}^{n}$-action on $\mathbf{T}^{1} \Phi_{k}: y \rightarrow y+\rho \cdot k$, which leaves invariant the Lebesgue measure, and to the integrable function on $\mathbf{T}^{1}$

$$
f(y)=\int_{[0,1]^{n}} \eta(x, u(x, y)) \cdot \nabla_{x} u(x, y) \mathrm{d} x
$$

getting

$$
\begin{aligned}
\lim _{R \rightarrow \infty} \frac{1}{\#\left\{k \in \mathbf{Z}^{n}:|k| \leq R\right\}} \sum_{|k| \leq R} \int_{[0,1]^{n}} \eta(x, u(x, y+\rho \cdot k)) \nabla_{x} u(x, y+\rho \cdot k) \mathrm{d} x=\int_{\mathbf{T}^{1}} f(y) \mathrm{d} y & =\int_{\mathbf{T}^{n+1}} \eta(x, u(x, y)) \cdot \nabla_{x} u(x, y) \mathrm{d} x \mathrm{~d} y
\end{aligned}
$$

which implies the existence of the limit in (1.1).

If $u \in M_{\rho}$ is not recurrent, then $u$ is heteroclinic between the two recurrent elements $u_{1}$ and $u_{2}$, and the same argument as in the rational case applies.

With our choice of the basis, if $u \in M_{\rho}$, then $\rho_{T_{u}}=(\rho, 1)$. To show this, let $\eta$ be a closed $n$-form on $\mathbf{T}^{n+1}$; we can write

$$
\eta=\sum_{i=1}^{n+1} c_{i} \widehat{\mathrm{d} x_{i}}+\mathrm{d} \psi
$$

where $c_{i} \in \mathbf{R}$. For the limit of the exact form $\mathrm{d} \psi$, we use Stokes:

$$
\begin{aligned}
\lim _{R \rightarrow \infty} \frac{1}{|B(0, R)|}\left|\int_{B(0, R)} \mathrm{d} \psi(x, u(x)) \cdot \nabla u(x) \mathrm{d} x\right| & =\lim _{R \rightarrow \infty} \frac{1}{|B(0, R)|}\left|\int_{\left.\operatorname{graph}(u)\right|_{B(0, R)}} \mathrm{d} \psi\right| \\
& =\lim _{R \rightarrow \infty} \frac{1}{|B(0, R)|}\left|\int_{\left.\operatorname{graph}(u)\right|_{\partial B(0, R)}} \psi\right| \leq \lim _{R \rightarrow \infty} C \frac{R^{n-1}}{R^{n}}=0
\end{aligned}
$$


where the inequality comes from the fact that $\psi$, being a periodic $(n-1)$-form on $\mathbf{R}^{n+1}$, is bounded. As a side result, we have that $T_{u}$ is closed. For the limit of the constant form $c$, we set $w(x)=\rho \cdot x$; we have that

$$
\begin{array}{r}
\lim _{R \rightarrow \infty} \frac{1}{|B(0, R)|}\left|\int_{B(0, R)}\left(\sum_{i=1}^{n+1} c_{i} \widehat{\mathrm{d} x_{i}}\right) \cdot(\nabla u(x)-\nabla w(x))\right|=\lim _{R \rightarrow \infty} \frac{1}{|B(0, R)|}\left|\sum_{i=1}^{n+1} c_{i} \int_{B(0, R)} \partial_{i}(u(x)-w(x)) \mathrm{d} x\right| \\
=\lim _{R \rightarrow \infty} \frac{1}{|B(0, R)|} \mid \sum_{i=1}^{n} c_{i} \int_{B^{\prime}(0, R)} \mathrm{d} x_{1} \ldots \mathrm{d} x_{i-1} \mathrm{~d} x_{i+1} \ldots \mathrm{d} x_{n}\left[(u-w)\left(x_{1}, \ldots, x_{i-1}, \sqrt{R^{2}-\left|x^{\prime}\right|^{2}}, x_{i+1}, \ldots, x_{n}\right)\right. \\
\left.-(u-w)\left(x_{1}, \ldots, x_{i-1},-\sqrt{R^{2}-\left|x^{\prime}\right|^{2}}, x_{i+1}, \ldots, x_{n}\right)\right] \mid \leq \lim _{R \rightarrow \infty} C \frac{R^{n-1}}{R^{n}}=0 \quad(1.4
\end{array}
$$

where $B^{\prime}(0, R)$ denotes the ball of radius $R$ in $\mathbf{R}^{n-1}$ and $x^{\prime}=\left(x_{1}, \ldots, x_{i-1}, x_{i+1}, \ldots, x_{n}\right)$; the second equality of the formula above is Fubini, the inequality follows from (3) in the introduction. An easy calculation shows that

$$
\lim _{R \rightarrow \infty} \frac{1}{|B(0, R)|} \int_{B(0, R)}\left(\sum_{i=1}^{n+1} c_{i} \widehat{\mathrm{d} x_{i}}\right) \cdot \nabla w(x) \mathrm{d} x=\sum_{i=1}^{n} c_{i} \rho_{i}+c_{n+1}
$$

By (1.3), (1.4) and (1.5) we get that, if $\eta$ is as above, then

$$
T_{u}(\eta)=\sum_{i=1}^{n} c_{i} \rho_{i}+c_{n+1}
$$

or $\rho_{T_{u}}=(\rho, 1)$, which is what we wanted to prove.

\section{A mean action for currents}

Let $\Lambda_{n}\left(\mathbf{R}^{n+1}\right)$ denote the set of $n$-vectors of $\mathbf{R}^{n+1}$. Since the forms $\left\{\widehat{\mathrm{d} x_{i}}\right\}_{i=1}^{n+1}$ are a base of $\Lambda^{n}\left(\mathbf{R}^{n+1}\right)$, the dual space of $\Lambda_{n}\left(\mathbf{R}^{n+1}\right)$, they induce a dual base $\left\{e_{i}\right\}_{i=1}^{n+1}$ on $\Lambda_{n}\left(\mathbf{R}^{n+1}\right)$; the Lagrangian $\mathcal{L}$ of the introduction induces immediately a Lagrangian $\tilde{\mathcal{L}}$ on $\mathbf{R}^{n+1} \times \Lambda_{n}\left(\mathbf{R}^{n+1}\right)$ by

$$
\tilde{\mathcal{L}}\left(x, u, p_{1} e_{1}+\ldots+p_{n} e_{n}+p_{n+1} e_{n+1}\right)=\left\{\begin{array}{rlll}
\mathcal{L}\left(x, u, p_{1}, \ldots, p_{n}\right) & \text { if } & p_{n+1}=1 \\
+\infty & \text { if } & p_{n+1} \neq 1
\end{array}\right.
$$

For the standard duality coupling between $\Lambda_{n}\left(\mathbf{R}^{n+1}\right)$ and $\Lambda^{n}\left(\mathbf{R}^{n+1}\right)$ we can define the Legendre transform of $\tilde{\mathcal{L}}$ :

$$
\begin{gathered}
\tilde{H}: \mathbf{T}^{n+1} \times \Lambda^{n}\left(\mathbf{R}^{n+1}\right) \rightarrow \mathbf{R} \\
\tilde{H}(x, u, \omega)=\sup _{p}\{\langle p, \omega\rangle-\tilde{\mathcal{L}}(x, u, p)\} .
\end{gathered}
$$

Since $\tilde{\mathcal{L}}=+\infty$ outside the affine plane $p_{n+1}=1$, we have that

$$
\tilde{H}\left(x, x_{n+1}, \sum_{i=1}^{n+1} c_{i} \widehat{\mathrm{d} x_{i}}\right)=H\left(x, x_{n+1}, \sum_{i=1}^{n} c_{i} \widehat{\mathrm{d} x_{i}}\right)+c_{n+1} .
$$

Let now $T$ be a $n$-current of finite mass; it is well-known that

$$
T=X \wedge \mu
$$

where $\mu$ is a measure on $\mathbf{T}^{n+1}$ and

$$
X: \mathbf{T}^{n+1} \rightarrow \Lambda_{n}\left(\mathbf{R}^{n+1}\right)
$$


is a Borel vector field. This parameterization is not unique: for instance, if $f, \frac{1}{f} \in L^{\infty}\left(\mathbf{T}^{n+1}, \mu\right)$, then we also have

$$
T=(X f) \wedge\left(\frac{1}{f} \mu\right)
$$

To a current $T$ we associate its component along $\mathbf{T}^{n+1}$, which is the measure $\mu_{T}$ on $\mathbf{T}^{n+1}$ defined by

$$
\int_{\mathbf{T}^{n+1}} f\left(x, x_{n+1}\right) \mathrm{d} \mu_{T}\left(x, x_{n+1}\right)=T\left(f \widehat{\mathrm{d} x_{n+1}}\right) \quad \forall f \in C\left(\mathbf{T}^{n+1}\right) .
$$

Choosing $f \equiv 1$, we see that $\mu_{T}\left(\mathbf{T}^{n+1}\right)$ is the $(n+1)$-th component of $\rho_{T}$.

Let $\Omega_{k}^{0}$ denote the space of continuous $k$-forms on $\mathbf{T}^{n+1}$; if $\omega \in \Omega_{n}^{0}$, let $\omega^{x}$ denote the projection of $\omega$ on the space generated by $\widehat{\mathrm{d} x_{1}}, \ldots, \widehat{\mathrm{d} x_{n}}$, and let $\omega^{u}$ denote the component of $\omega$ along $\widehat{\mathrm{d} x_{n+1}}$. The following proposition is taken from [6].

Proposition 1.1. Let $T$ be a closed $n$-current on $\mathbf{T}^{n+1}$, and let us suppose that the measure $\mu_{T}$ defined by (1.6) is a probability measure. Then all the $A_{i}(T)$ defined below are equal:

$$
\begin{aligned}
& A_{1}(T)=\sup _{\omega \in \Omega_{n}^{0}}\left\{T\left(\omega^{x}\right)-\int_{\mathbf{T}^{n+1}} H\left(x, x_{n+1}, \omega^{x}\right) \mathrm{d} \mu_{T}\left(x, x_{n+1}\right)\right\} \\
& A_{2}(T)=\sup _{\omega \in \Omega_{n}^{0}}\left\{T(\omega)-\int_{\mathbf{T}^{n+1}}\left[H\left(x, x_{n+1}, \omega^{x}\right)+\omega^{u}\right] \mathrm{d} \mu_{T}\left(x, x_{n+1}\right)\right\} \\
& A_{3}(T)=\sup _{\omega \in \Omega_{n}^{0}}\left\{T(\omega)-\sup _{\left(x, x_{n+1}\right) \in \mathbf{T}^{n+1}}\left[H\left(x, x_{n+1}, \omega^{x}\right)+\omega^{u}\right]\right\} \\
& A_{4}(T)=\sup \left\{T(\omega): \omega \in \Omega_{n}^{0}, \quad \omega^{u}+H\left(x, x_{n+1}, \omega^{x}\right) \leq 0\right\} \\
& A_{5}(T)=\sup \left\{T(\omega): \omega \in \Omega_{n}^{0}, \quad \omega^{u}+H\left(x, x_{n+1}, \omega^{x}\right)=0\right\} .
\end{aligned}
$$

Proof. By (1.6) we have that

Thus

$$
T\left(\omega^{u} \widehat{\mathrm{d} x_{n+1}}\right)-\int_{\mathbf{T}^{n+1}} \omega^{u} \mathrm{~d} \mu_{T}=0 .
$$

Since $\mu_{T}$ is a probability measure, we get that

$$
A_{1}(T)=A_{2}(T) .
$$

$$
A_{3}(T) \leq A_{2}(T)
$$

We also note that

$$
A_{5}(T) \leq A_{4}(T) \leq A_{3}(T)
$$

where the first inequality follows since we are taking the sup on a smaller set and the second one is obvious. For $\omega=\left(\omega^{x}, \omega^{u}\right) \in \Omega_{n}^{0}$ we set $\tilde{\omega}_{u}=-H\left(x, x_{n+1}, \omega^{x}\right)$ and we see that

$$
\begin{aligned}
T\left(\omega^{x}+\omega^{u} \widehat{\mathrm{d} x_{n+1}}\right)-\int_{\mathbf{T}^{n+1}}\left[H\left(x, x_{n+1}, \omega^{x}\right)+\omega^{u}\right] \mathrm{d} \mu_{T} & =T\left(\omega^{x}+\tilde{\omega}^{u} \widehat{\mathrm{d} x_{n+1}}\right)-\int_{\mathbf{T}^{n+1}}\left[H\left(x, x_{n+1}, \omega^{x}\right)+\tilde{\omega}^{u}\right] \mathrm{d} \mu_{T} \\
& \leq A_{5}(T)
\end{aligned}
$$

The equality comes from (1.7) applied to $\omega^{u}$ and $\tilde{\omega}^{u}$ and the inequality from the fact that $H\left(x, x_{n+1}, \omega^{x}\right)+\tilde{\omega}^{u}=$ 0. Since (1.8) holds $\forall \omega \in \Omega_{n}^{0}$, we have that

$$
A_{2}(T) \leq A_{5}(T)
$$

and this ends the proof. 
Notation. From now on we shall call $A(T)$ the common value of the $A_{i}(T), 1 \leq i \leq 5$.

We won't address the question whether the minimum of $A$ on all closed currents of rotation number $\rho$ is the current induced by an element of $M_{\rho}$; we have introduced $A(T)$ only to have a compact notation for the limit in $(5)$.

We shall need another formulation, taken from [8]. Given a probability measure $\sigma$ on $\mathbf{T}^{n+1}$ and a closed current $T$, we define

$$
A_{6}(\sigma, T)=\sup _{\omega \in \Omega_{n}^{0}}\left\{\int_{\mathbf{T}^{n+1}}-\tilde{H}\left(x, x_{n+1}, \omega\left(x, x_{n+1}\right)\right) \mathrm{d} \sigma\left(x, x_{n+1}\right)+T(\omega)\right\}
$$

or equivalently

$$
A_{6}(\sigma, T)=\sup _{\alpha, \omega}\left\{\int_{\mathbf{T}^{n+1}} \alpha\left(x, x_{n+1}\right) \mathrm{d} \sigma\left(x, x_{n+1}\right)+T(\omega)\right\}
$$

where the sup is taken over all the couples $(\alpha, \omega) \in C\left(\mathbf{T}^{n+1}\right) \times \Omega_{n}^{0}$ satisfying

$$
\alpha\left(x, x_{n+1}\right)+\tilde{H}\left(x, x_{n+1}, \omega\left(x, x_{n+1}\right)\right) \leq 0 \quad \forall\left(x, x_{n+1}\right) \in \mathbf{T}^{n+1} .
$$

If the measure $\mu_{T}$ defined by (1.6) is a probability measure, we obviously have

$$
A_{6}\left(\mu_{T}, T\right)=A_{2}(T)=A(T)
$$

We only sketch the proof of the following two lemmas, since they are identical to [8].

Lemma 1.2. There is $C \in \mathbf{R}$ such that, for any probability measure $\sigma$ and any current of finite mass $T$, we have $A_{6}(\sigma, T) \geq C$. If $A_{6}(\sigma, T)<+\infty$, then there is a Borel $n$-vector field $X \in L^{1}\left(\mathbf{T}^{n+1}, \sigma\right)$ such that $T=X \wedge \sigma$. Moreover, $X_{n+1}=1 \sigma$ a.e.

Proof. Our hypotheses on $\mathcal{L}$ imply that $\mathcal{L} \geq C$; by the definition of Legendre transform we have that

$$
\tilde{H}\left(x, x_{n+1}, 0\right)=\sup \left\{-\tilde{\mathcal{L}}\left(x, x_{n+1}, p\right): p \in \Lambda_{n}\left(\mathbf{R}^{n+1}\right)\right\} \leq-C .
$$

As a consequence, the couple $\alpha \equiv C, \omega \equiv 0$ is admissible for the sup in the definition of $A_{6}$, and thus

$$
A_{6}(\sigma, T) \geq C
$$

Let us now assume that $A_{6}(\sigma, T)<+\infty$. It is a standard fact (see for instance [8]) that $T$ can be parameterized as $T=X \wedge \tilde{\sigma}$, with $\tilde{\sigma}$ a probability measure on $\mathbf{T}^{n+1}$, and $\|X\|_{\Lambda_{n}\left(\mathbf{R}^{n+1}\right)}=M(T) \tilde{\sigma}$ a.e., where $M(T)$ denotes the mass of $T$. The mass norm $\|X\|_{\Lambda_{n}\left(\mathbf{R}^{n+1}\right)}$ and its dual $\|\omega\|_{\Lambda^{n}\left(\mathbf{R}^{n+1}\right)}$ are defined in the standard way, as in [24], Chapter II, p. 10. We write $\tilde{\sigma}=\tilde{\sigma}^{a}+\tilde{\sigma}^{s}$, with $\tilde{\sigma}^{a} \ll \sigma$ and $\tilde{\sigma}^{s} \perp \sigma$; we must show that $\tilde{\sigma}^{s}=0$.

We rewrite $A_{6}$ as

$$
A_{6}(\sigma, T)=\sup _{\alpha, \omega} \int_{\mathbf{T}^{n+1}} \alpha\left(x, x_{n+1}\right) \mathrm{d} \sigma\left(x, x_{n+1}\right)+\int_{\mathbf{T}^{n+1}} \omega\left(x, x_{n+1}\right) \cdot X\left(x, x_{n+1}\right) \mathrm{d} \tilde{\sigma}\left(x, x_{n+1}\right) .
$$

We can find a Borel $n$-form $\tilde{\omega}$ which, for $\tilde{\sigma}$ a.e. $\left(x, x_{n+1}\right)$, satisfies

$$
\left\{\begin{array}{c}
\tilde{\omega}\left(x, x_{n+1}\right) \cdot X\left(x, x_{n+1}\right)=\left\|X\left(x, x_{n+1}\right)\right\|_{\Lambda_{n}\left(\mathbf{R}^{n+1}\right)}=M(T) \\
\|\tilde{\omega}\|_{L^{\infty}\left(\mathbf{T}^{n+1}\right)} \leq 1 .
\end{array}\right.
$$

Since the unit ball of the mass norm is not strictly convex, $\tilde{\omega}\left(x, x_{n+1}\right)$ is not unique; but, since the set of the $\tilde{\omega}\left(x, x_{n+1}\right)$ of mass norm 1 and satisfying the equality of (1.12) is convex, it is not hard to find a measurable selection. 
Let us call $B$ the set on which $\tilde{\sigma}^{s}$ is concentrated. Let us set $\omega\left(x, x_{n+1}\right)=\tilde{\omega}\left(x, x_{n+1}\right) \cdot 1_{B}\left(x, x_{n+1}\right)$. Using Lusin's theorem with respect to the measure $\sigma+\tilde{\sigma}^{s}$ on $\mathbf{T}^{n+1}$, we can find continuous forms $\omega_{\epsilon}$ such that

$$
\left\{\begin{array}{l}
\left\|\omega_{\epsilon}\right\|_{L^{\infty}\left(\mathbf{T}^{n+1}\right) \leq 1} \\
\omega_{\epsilon} \rightarrow \omega \sigma+\tilde{\sigma}^{s} \text { a.e. } \\
\left(\sigma+\tilde{\sigma}^{s}\right)\left\{\left(x, x_{n+1}\right) \in \mathbf{T}^{n+1}: \omega_{\epsilon}\left(x, x_{n+1}\right) \neq \omega\left(x, x_{n+1}\right)\right\}<\epsilon .
\end{array}\right.
$$

Let us define $\alpha_{\epsilon}\left(x, x_{n+1}\right)=-\tilde{H}\left(x, x_{n+1}, \omega_{\epsilon}\left(x, x_{n+1}\right)\right)$. Since $\tilde{H}$ is continuous, also $\alpha_{\epsilon}$ is such and converges, $\sigma+\tilde{\sigma}^{s}$ a.e., to $\alpha\left(x, x_{n+1}\right)=-\tilde{H}\left(x, x_{n+1}, \omega\left(x, x_{n+1}\right)\right)$. Moreover, the couple $\left(\alpha_{\epsilon}, \omega_{\epsilon}\right)$ is admissible for the sup in the definition of $A_{6}$. This and (1.11) implies the first inequality in the following formula:

$$
\begin{aligned}
A_{6}(\sigma, T) & \geq \int_{\mathbf{T}^{n+1}} \alpha_{\epsilon}\left(x, x_{n+1}\right) \mathrm{d} \sigma\left(x, x_{n+1}\right)+\int_{\mathbf{T}^{n+1}} \omega_{\epsilon}\left(x, x_{n+1}\right) \cdot X\left(x, x_{n+1}\right) \mathrm{d} \tilde{\sigma}\left(x, x_{n+1}\right) \\
& =\int_{\left\{\omega_{\epsilon} \neq \omega\right\}} \alpha_{\epsilon} \mathrm{d} \sigma-\int_{\left\{\omega_{\epsilon}=\omega\right\}} \tilde{H}\left(x, x_{n+1}, \omega\left(x, x_{n+1}\right)\right) \mathrm{d} \sigma+\int_{\mathbf{T}^{n+1}} \omega_{\epsilon} \cdot X \mathrm{~d} \tilde{\sigma} \\
& \geq-\left\|\alpha_{\epsilon}\right\|_{\infty} \sigma\left\{\omega_{\epsilon} \neq \omega\right\}-\int_{\left\{\omega_{\epsilon}=\omega\right\}} \tilde{H}\left(x, x_{n+1}, \omega\left(x, x_{n+1}\right)\right) \mathrm{d} \sigma+\int_{\mathbf{T}^{n+1}} \omega_{\epsilon} \cdot X \mathrm{~d} \tilde{\sigma} \\
& \geq-C_{1} \epsilon+C+\int_{\mathbf{T}^{n+1}} \omega_{\epsilon} \cdot X \mathrm{~d} \tilde{\sigma} .
\end{aligned}
$$

The equality comes from the definition of $\alpha_{\epsilon}$, and the only inequality which need explanation is the last one. For the estimate on $\left\|\alpha_{\epsilon}\right\| \sigma\left\{\omega_{\epsilon} \neq \omega\right\}$, we have used the fact that $\alpha_{\epsilon}=-\tilde{H}\left(x, x_{n+1}, \omega_{\epsilon}\right)$, we have set

$$
C_{1}=\sup \left\{\left|\tilde{H}\left(x, x_{n+1}, p\right)\right|:\|p\| \leq 1,\left(x, x_{n+1}\right) \in \mathbf{T}^{n+1}\right\}
$$

and we have used (1.13). For the estimate on the integral of $\tilde{H}$, which we want independent on the norm of $\omega_{\epsilon}$, we have used the fact that

$$
\begin{aligned}
\int_{\left\{\omega_{\epsilon}=\omega\right\}} \tilde{H}\left(x, x_{n+1}, \omega\left(x, x_{n+1}\right)\right) \mathrm{d} \sigma & =\int_{\left\{\omega_{\epsilon}=\omega\right\} \cap B} \tilde{H}\left(x, x_{n+1}, \omega\left(x, x_{n+1}\right)\right) \mathrm{d} \sigma+\int_{\left\{\omega_{\epsilon}=\omega\right\} \backslash B} \tilde{H}\left(x, x_{n+1}, \omega\left(x, x_{n+1}\right)\right) \mathrm{d} \sigma \\
& =\int_{\left\{\omega_{\epsilon}=\omega\right\} \backslash B} \tilde{H}\left(x, x_{n+1}, 0\right) \mathrm{d} \sigma \leq-C
\end{aligned}
$$

because $\sigma(B)=0,\left.\omega\right|_{B^{c}}=0$ and (1.10) holds. Passing to the limit as $\epsilon \rightarrow 0$ in (1.14), and taking into account that

$$
\lim _{\epsilon \rightarrow 0} \int_{\mathbf{T}^{n+1}} \omega_{\epsilon} \cdot X \mathrm{~d} \tilde{\sigma}=\int_{\mathbf{T}^{n+1}} \omega \cdot X \mathrm{~d} \tilde{\sigma}
$$

by (1.13) and dominated convergence, we get that

$$
A_{6}(\sigma, T) \geq C+\int_{\mathbf{T}^{n+1}} \omega \cdot X \mathrm{~d} \tilde{\sigma}=C+\int_{B} \tilde{\omega} \cdot X \mathrm{~d} \tilde{\sigma}=C+M(T) \tilde{\sigma}^{s}\left(\mathbf{T}^{n+1}\right)
$$

where the first equality comes from the fact that $\omega=1_{B} \tilde{\omega}$ and the last one from (1.12). Now it suffices to note that, for any $k \in \mathbf{N}$, one can repeat the argument above with $k \omega$ instead of $\omega$; since the constant $C$ of (1.10) does not depend on $k$ but only on $H$, we get that

$$
A_{6}(\sigma, T) \geq C+k M(T) \tilde{\sigma}^{s}\left(\mathbf{T}^{n+1}\right) .
$$

Letting $k \rightarrow+\infty$, we get $\tilde{\sigma}^{s}=0$, i.e. $T=X \wedge \sigma$, which is what we wanted. 
To prove the last assertion of the thesis, we suppose by contradiction that there is $B \subset \mathbf{T}^{n+1}$ with $\sigma(B)>0$ such that $X_{n+1}>1+\epsilon$ on $B$. We define the form $\omega_{\lambda}=\lambda 1_{B} \widehat{\mathrm{d} x_{n+1}}$ and we see that

$$
\begin{aligned}
A_{6}(\sigma, T) & \geq \sup _{\lambda>0}\left\{\int_{\mathbf{T}^{n+1}}-\tilde{H}\left(x, x_{n+1}, \omega_{\lambda}\right) \mathrm{d} \sigma+T\left(\omega_{\lambda}\right)\right\} \\
& =\sup _{\lambda>0}\left\{\int_{\mathbf{T}^{n+1}}-H\left(x, x_{n+1}, \omega_{\lambda}^{x}\right) \mathrm{d} \sigma-\int_{\mathbf{T}^{n+1}} \omega_{\lambda}^{u} \mathrm{~d} \sigma+T\left(\omega_{\lambda}\right)\right\} \\
& =\sup _{\lambda>0}\left\{\int_{\mathbf{T}^{n+1}}-H\left(x, x_{n+1}, 0\right) \mathrm{d} \sigma-\int_{\mathbf{T}^{n+1}} \lambda 1_{B} \mathrm{~d} \sigma+\int_{\mathbf{T}^{n+1}} \lambda 1_{B} X_{n+1} \mathrm{~d} \sigma\right\} \\
& \geq \sup _{\lambda>0}\left\{\int_{\mathbf{T}^{n+1}}-H\left(x, x_{n+1}, 0\right) \mathrm{d} \sigma+\int_{B} \lambda \epsilon \mathrm{d} \sigma\right\}=+\infty .
\end{aligned}
$$

Using Lusin to smooth $\omega_{\lambda}$, we see that

$$
A_{6}(\sigma, T)=+\infty
$$

contrary to the hypothesis. By a similar argument, $X_{n+1}>1-\epsilon \sigma$ a.e.; thus, $X_{n+1}=1 \sigma$ a.e., which ends the proof.

Lemma 1.3. Let $T=X \wedge \sigma$ with $X \in L^{1}(\sigma)$ and $X_{n+1}=1 \sigma$ a.e. Then

$$
A_{6}(\sigma, T)=\int_{\mathbf{T}^{n+1}} \tilde{\mathcal{L}}\left(x, x_{n+1}, X\left(x, x_{n+1}\right)\right) \mathrm{d} \sigma\left(x, x_{n+1}\right) .
$$

Proof. We recall that

$$
\tilde{\mathcal{L}}\left(x, x_{n+1}, p\right)+\tilde{H}\left(x, x_{n+1}, \omega\right) \geq \omega \cdot p
$$

with equality only when $\omega$ is the Legendre transform of $p$. Thus, for any couple $(\alpha, \omega)$ such that

$$
\alpha\left(x, x_{n+1}\right)+\tilde{H}\left(x, x_{n+1}, \omega\left(x, x_{n+1}\right)\right) \leq 0
$$

we get that

$$
\int_{\mathbf{T}^{n+1}}\left[\alpha\left(x, x_{n+1}\right)+\omega\left(x, x_{n+1}\right) \cdot X\left(x, x_{n+1}\right)\right] \mathrm{d} \sigma\left(x, x_{n+1}\right) \leq \int_{\mathbf{T}^{n+1}} \tilde{\mathcal{L}}\left(x, x_{n+1}, X\left(x, x_{n+1}\right)\right) \mathrm{d} \sigma\left(x, x_{n+1}\right) .
$$

Passing to the sup, this implies

$$
A_{6}(\sigma, T) \leq \int_{\mathbf{T}^{n+1}} \tilde{\mathcal{L}}\left(x, x_{n+1}, X\left(x, x_{n+1}\right)\right) \mathrm{d} \sigma\left(x, x_{n+1}\right) .
$$

Now we consider $\omega\left(x, x_{n+1}\right)$, the Legendre transform of $X\left(x, x_{n+1}\right)$. We know from the hypotheses that $X_{n+1}=1$; in particular, this implies that the Legendre transform of $X$ is not unique, because if $\omega$ yields equality in (1.15), then also $\omega+\lambda \widehat{\mathrm{d} x_{n+1}}$ yields equality. We choose the $\omega$ with $\omega_{n+1}=0$; equivalently, $\omega=\left(\omega^{x}, 0\right)$ with $\omega^{x}$ the Legendre transform of $\left(X_{1}, \ldots, X_{n}\right)$ by

$$
:\left(X_{1}, \ldots, X_{n}\right) \rightarrow \mathcal{L}\left(x, x_{n+1}, X_{1}, \ldots, X_{n}, 1\right) .
$$

Let now $\omega_{k}$ be $\omega$ truncated to 0 when $\|\omega\|>k$. Since $X$ is Borel, by the continuity of the Legendre transform we have that $\omega$ too is Borel; in particular, $\omega_{k} \in L^{\infty}(\sigma)$. Moreover, defining

$$
A_{k}=\left\{\left(x, x_{n+1}\right):\left|\omega\left(x, x_{n+1}\right)\right| \leq k\right\}
$$


we have that $1_{A_{k}} \rightarrow 1 \sigma$ a.e.; this is because $X \in L^{1}(\sigma)$ is finite $\sigma$ a.e. and $\omega$, being the Legendre transform of $X$, has the same property. Now we take

$$
\left(\omega_{k}, \alpha_{k}=-\tilde{H}\left(x, x_{n+1}, \omega_{k}\right)\right)
$$

as an admissible couple in the sup defining $A_{6}$ and we get

$$
\begin{aligned}
A_{6}(\sigma, T) \geq & \int_{\mathbf{T}^{n+1}}\left[-\tilde{H}\left(x, x_{n+1}, \omega_{k}\left(x, x_{n+1}\right)\right)+\omega_{k} \cdot X\right] \mathrm{d} \sigma \\
= & \int_{A_{k}}\left[-\tilde{H}\left(x, x_{n+1}, \omega_{k}\left(x, x_{n+1}\right)\right)+\omega_{k} \cdot X\right] \mathrm{d} \sigma \\
& +\int_{A_{k}^{c}}-\tilde{H}\left(x, x_{n+1}, 0\right) \mathrm{d} \sigma\left(x, x_{n+1}\right) \\
= & \int_{\mathbf{T}^{n+1}} \tilde{\mathcal{L}}\left(x, x_{n+1}, X\left(x, x_{n+1}\right)\right) 1_{A_{k}}\left(x, x_{n+1}\right) \mathrm{d} \sigma\left(x, x_{n+1}\right) \\
& -\int_{\mathbf{T}^{n+1}} \tilde{H}\left(x, x_{n+1}, 0\right)\left[1-1_{A_{k}}\left(x, x_{n+1}\right)\right] \mathrm{d} \sigma\left(x, x_{n+1}\right)
\end{aligned}
$$

where the last equality comes from the fact that $\omega$ is the Legendre transform of $X$. We let now $k \rightarrow+\infty$; we apply Fatou's lemma to the first term on the right and note that, since $1_{A_{k}} \rightarrow 1$ and $\tilde{H}\left(x, x_{n+1}, 0\right)$ is bounded, dominated convergence applies to the second term. Thus

$$
A_{6}(\sigma, T) \geq \int_{\mathbf{T}^{n+1}} \tilde{\mathcal{L}}\left(x, x_{n+1}, X\right) \mathrm{d} \sigma
$$

which, together with (1.16), yields the thesis.

Now, if $T=X \wedge \sigma$ and $\nu$ is the probability measure on $\mathbf{T}^{n+1} \times \Lambda_{n}\left(\mathbf{R}^{n+1}\right)$ which is the push-forward of $\sigma$ by $X$, Lemma 1.3 implies that

$$
A_{6}(\sigma, T)=\int_{\mathbf{T}^{n+1} \times \Lambda\left(\mathbf{R}^{n+1}\right)} \mathcal{L}\left(x, x_{n+1}, p\right) \mathrm{d} \nu\left(x, x_{n+1}, p\right) .
$$

Let now $u \in M_{\rho}$, and let $\nu_{R}$ be the measure on $\mathbf{T}^{n+1} \times \Lambda_{n}\left(\mathbf{R}^{n+1}\right)$ defined by

$$
\mu_{R}(\phi)=\frac{1}{|B(0, R)|} \int_{B(0, R)} \phi(x, u, \nabla u) \mathrm{d} x
$$

for any continuous $\phi$ compactly supported in $\mathbf{T}^{n+1} \times \Lambda_{n}\left(\mathbf{R}^{n+1}\right)$. It is easy to see that $\nu_{R}$ is positive and that $\nu_{R}\left(\mathbf{T}^{n+1} \times \Lambda_{n}\left(\mathbf{R}^{n+1}\right)\right)=1$ (i.e. $\nu_{R}$ is a probability measure); moreover, (3) implies that the support of all the $\nu_{R}$ is contained in the bounded set $\mathbf{T}^{n+1} \times B\left(0, M_{1}(\rho)\right)$. In particular, we can find $R_{k} \rightarrow+\infty$ such that $\nu_{R_{k}}$ converges, up to a subsequence, to a compactly supported $\nu$. Thus we have that

$$
\int_{\mathbf{T}^{n+1} \times \Lambda_{n}\left(\mathbf{R}^{n+1}\right)} f(x, u, p) \mathrm{d} \nu(x, u, p)=\lim _{k \rightarrow+\infty} \frac{1}{\left|B\left(0, R_{k}\right)\right|} \int_{B\left(0, R_{k}\right)} f(x, u, \nabla u) \mathrm{d} x
$$

for all continuous functions vanishing at infinity; actually, since $\nu$ and $\nu_{R_{k}}$ are supported on the same compact set, it holds for all continuous functions.

Now $\nu$ induces a current $T$ by

$$
T(\omega)=\int_{\mathbf{T}^{n+1} \times \Lambda_{n}\left(\mathbf{R}^{n+1}\right)} \omega(x, u) \cdot p \mathrm{~d} \nu(x, u, p)
$$


for any $n$-form $\omega$. We have that $T=T_{u}$, because by definition

$$
T_{u}(\psi)=\lim _{R \rightarrow+\infty} \frac{1}{|B(0, R)|} \int_{B(0, R)} \psi(x, u) \cdot \nabla u \mathrm{~d} x
$$

for continuous $n$-forms $\psi$, i.e. on a subspace of $C\left(\mathbf{T}^{n+1} \times \Lambda_{n}\left(\mathbf{R}^{n+1}\right)\right)$. For $u$ as above, we can define by $(1.2)$ a field of $n$-vectors $X$ on the graph of $u$; since by [17] this field is Lipschitz, we can extend it to the closure of the graph of $u$ in $\mathbf{T}^{n+1}$. It is easy to check that $T_{u}=X \wedge \mu_{T_{u}}$ for the measure $\mu_{T_{u}}$ defined in (1.5), and that $\mu_{T_{u}}$ is the Lebesgue measure. Moreover, $\nu$ is the push-forward of $\mu_{T_{u}}$ by $X$; thus, by (1.17) and (1.18) with $f=\mathcal{L}$ we get

$$
\begin{aligned}
A_{6}\left(\sigma, T_{u}\right) & =\int_{\mathbf{T}^{n+1} \times \Lambda_{n}\left(\mathbf{R}^{n+1}\right)} \mathcal{L}\left(x, x_{n+1}, p\right) \mathrm{d} \nu\left(x, x_{n+1}, p\right) \\
& =\lim _{R \rightarrow+\infty} \frac{1}{|B(0, R)|} \int_{B(0, R)} \mathcal{L}(x, u, \nabla u) \mathrm{d} x=\beta(\rho)
\end{aligned}
$$

where $\beta$ has been defined in (5) of the introduction. We shall extend $\beta$ to all $H_{n}\left(\mathbf{T}^{n+1}\right)$ by

$$
\tilde{\beta}\left(\rho, \rho_{n+1}\right)=\left\{\begin{array}{lll}
\beta(\rho) & \text { if } & \rho_{n+1}=1 \\
+\infty & \text { if } & \rho_{n+1} \neq 1 .
\end{array}\right.
$$

We shall call $-\tilde{\alpha}$ the polar of $\tilde{\beta}$; it is clear that $\tilde{\alpha}\left(c, c_{n+1}\right)=\alpha(c)-c_{n+1}$ with $c \in H$, where $H$ is defined by

$$
H=H^{n}\left(\mathbf{T}^{n+1}\right) \cap\left\{c_{n+1}=0\right\} .
$$

The same calculation as in [15] now yields

$$
\alpha(c)=\min \left\{A(T)-T\left(\eta_{c}\right)\right\}
$$

where $\eta_{c}$ is a $n$-form representing $c$, and the minimum is over all currents induced by elements of $M_{\rho}$, with $(\rho, 1)$ varying in $H_{n}\left(\mathbf{T}^{n+1}\right)$. We recall that the minimum above is attained by the currents induced by the elements of $M_{-\alpha^{\prime}(c)}$.

\section{LAMinations in $M_{\rho}$ AND THE DIFFERENTIABILITy OF $\beta$}

Let $\mathcal{L}, H, \beta$ and $\alpha$ be as in the previous section; we want to study the differentiability of $\beta$ or, equivalently, the flats of $\alpha$. Before giving a precise statement, we recall some notions from [17] and [4]. First of all, we define the recurrent elements of $M_{\rho}$.

If $\rho \in \mathbf{Q}^{n}$, we say that $u \in M_{\rho}$ is recurrent if it is periodic:

$$
u(x+k)+j=u(x) \quad \text { if } \quad(k, j) \in \mathbf{Z}^{n} \times \mathbf{Z} \cap(\rho, 1)^{\perp} .
$$

If $\rho \notin \mathbf{Q}^{n}$, the recurrent elements of $M_{\rho}$ are, by definition, those in the one-parameter family $u_{\rho}(x, \lambda)$, where $u_{\rho}$ is built in the following way. There is a function $U_{\rho}: \mathbf{R}^{n+1} \rightarrow \mathbf{R}$ such that $U_{\rho}\left(x, x_{n+1}\right)-x_{n+1}$ has period 1 in $x_{1}, \ldots, x_{n+1}$, the map : $x_{n+1} \rightarrow U_{\rho}\left(x, x_{n+1}\right)$ is strictly monotone increasing and continuous from above, and

$$
u_{\rho}(x, \lambda)=U_{\rho}(x, \rho \cdot x+\lambda)
$$

belongs to $M_{\rho}$ for every value of $\lambda$. In other words, the self-map of $\mathbf{T}^{n+1}$ given by

$$
:\left(x, x_{n+1}\right) \rightarrow\left(x, U_{\rho}\left(x, x_{n+1}\right)\right)
$$


brings the foliation $x_{n+1}=\rho \cdot x+\lambda$ into the recurrent elements of $M_{\rho}$. Moreover, the function $U_{\rho}$ is unique in the following sense: if $U: \mathbf{R}^{n+1} \rightarrow \mathbf{R}$ is another function with the properties above, then $U\left(x, x_{n+1}\right)=$ $U_{\rho}\left(x, x_{n+1}+\beta\right)$ for some $\beta \in \mathbf{R}$.

It is proven in [17] that, for all $\rho \in \mathbf{R}^{n}, M_{\rho}$ contains recurrent elements.

Definition. We shall call $M_{\rho}^{\text {rec }}$ the set of all recurrent elements of $M_{\rho}$.

If $\rho \in \mathbf{R}^{n} \backslash \mathbf{Q}^{n}$, it follows from the monotonicity of $U_{\rho}$ that

$$
\lambda<\lambda^{\prime} \quad \text { iff } \quad u_{\rho}(x, \lambda)<u_{\rho}\left(x, \lambda^{\prime}\right) \quad \forall x \in \mathbf{R}^{n}
$$

i.e. $M_{\rho}^{r e c}$ is an ordered set. This is also true if $\rho \in \mathbf{Q}^{n}$ :

$$
\text { if } u, v \in M_{\rho}^{r e c} \text {, then either } u<v \quad \text { or } u \equiv v \text { or } u>v \text {. }
$$

An ordered subset of $M_{\rho}$ is called a lamination, and in general there are laminations of $M_{\rho}$ strictly containing $M_{\rho}^{r e c}$. We now recall the way they are classified in [4].

Let $u \in M_{\rho}$ and let us consider the set

$$
\Phi_{0}(u)=\left\{(k, j) \in \mathbf{Z}^{n} \times \mathbf{Z}: u(x+k)+j \geq u(x)\right\}
$$

Clearly, $\Phi_{0}(u)$ contains all the information on the directions in which $u$ increases; it is also clear that it is a semigroup. We want to explain the method used in [4] to characterize this semigroup by a sequence of mutually orthogonal vectors. We begin to note that, since $(0, j) \notin \Phi_{0}(u)$ if $j<0$,

(i) $\Phi_{0}(u) \neq \mathbf{Z}^{n} \times \mathbf{Z}$.

Moreover, by formula (2) of the introduction,

(ii) $\Phi_{0}(u) \cup-\Phi_{0}(u)=\mathbf{Z}^{n} \times \mathbf{Z}$.

A semigroup with these two properties determines uniquely an open half-space $V_{0}$ of $\mathbf{R}^{n+1}$ by

$$
V_{0} \cap\left(\mathbf{Z}^{n} \times \mathbf{Z}\right) \subset \Phi_{0}(u) \subset \bar{V}_{0} \cap\left(\mathbf{Z}^{n} \times \mathbf{Z}\right)
$$

In our case it is easy to see that

$$
V_{0}=\left\{\left(x, x_{n+1}\right) \in \mathbf{R}^{n} \times \mathbf{R}:\left\langle\left(x, x_{n+1}\right),(\rho, 1)\right\rangle>0\right\}
$$

We want to describe the elements of $\partial V_{0} \cap \Phi_{0}(u)$; thus, let $\operatorname{rat}(\rho, 1)$ denote the subspace of $\mathbf{R}^{n} \times \mathbf{R}$ generated by $\left(\mathbf{Z}^{n} \times \mathbf{Z}\right) \cap(\rho, 1)^{\perp}$, and let us define

$$
\Phi_{1}(u)=\Phi_{0}(u) \cap(\rho, 1)^{\perp}=\Phi_{0}(u) \cap \operatorname{rat}(\rho, 1)
$$

where the second equality comes from the fact that $\Phi_{0}(u) \subset\left(\mathbf{Z}^{n} \times \mathbf{Z}\right)$. Again by (2) we have that

$$
\Phi_{1}(u) \cup-\Phi_{1}(u)=\left(\mathbf{Z}^{n} \times \mathbf{Z}\right) \cap \operatorname{rat}(\rho, 1) .
$$

If $\Phi_{1}(u) \neq\left(\mathbf{Z}^{n} \times \mathbf{Z}\right) \cap \operatorname{rat}(\rho, 1)$, we can find as before a vector $v_{1}(u) \in \operatorname{rat}(\rho, 1)$ such that

$$
\Phi_{1}(u) \subset \operatorname{rat}(\rho, 1) \cap\left\{(k, j):\left\langle(k, j), v_{1}(u)\right\rangle \geq 0\right\} .
$$

In the terminology of [19], $u$ is a heteroclinic between two different elements of $M_{\rho}^{r e c}$, and $v_{1}(u)$ is the asymptotic direction of $u$. In general, if $\Phi_{i}(u)=\Phi_{i-1}(u) \cap \operatorname{rat} v_{i-1}(u)$ is strictly contained in

$$
\left(\mathbf{Z}^{n} \times \mathbf{Z}\right) \cap \operatorname{rat}(\rho, 1) \cap \operatorname{rat} v_{1}(u) \cap \ldots \cap \operatorname{rat} v_{i-1}(u)
$$


we can find $v_{i}(u)$ in the intersection above such that

$$
\Phi_{i}(u) \subset \operatorname{rat}(\rho, 1) \cap \operatorname{rat} v_{1}(u) \cap \ldots \cap \operatorname{rat} v_{i-1}(u) \cap\left\{(k, j):\left\langle(k, j), v_{i}(u)\right\rangle \geq 0\right\} .
$$

We can iterate until we come to a stop when

$$
\Phi_{l}(u)=\left(\mathbf{Z}^{n} \times \mathbf{Z}\right) \cap \operatorname{rat}(\rho, 1) \cap \operatorname{rat} v_{1}(u) \cap \ldots \cap \operatorname{rat} v_{l-1}(u) .
$$

We recall that $\operatorname{rat} v_{i-1}(u)$ is the space generated by $v_{i-1}(u)^{\perp} \cap\left(\mathbf{Z}^{n} \times \mathbf{Z}\right)$; since $v_{i}(u) \in$ rat $v_{i-1}(u)$, the vectors $v_{i}(u)$ are mutually orthogonal. In particular, at each step the dimension of the intersection in the formula above decreases at least by 1 , and eventually a stop is reached, say after $l=l(u)$ steps. In this way, we get the $l+1$ vectors

$$
\begin{gathered}
(\rho, 1), \quad v_{1}(u) \in \operatorname{rat}(\rho, 1), \quad v_{2}(u) \in \operatorname{rat}(\rho, 1) \cap \operatorname{rat} v_{1}(u), \ldots, \\
v_{l}(u) \in \operatorname{rat}(\rho, 1) \cap \operatorname{rat} v_{1}(u) \cap \ldots \cap \operatorname{rat} v_{l-1}(u) .
\end{gathered}
$$

By definition, these vectors satisfy

$$
\begin{gathered}
\left\{(k, j): u(x+k)+j \geq u(x),(k, j) \in \operatorname{rat}(\rho, 1) \cap \ldots \cap \operatorname{rat} v_{s}(u)\right\} \subset \\
\left\{(k, j) \in \operatorname{rat}(\rho, 1) \cap \ldots \cap \operatorname{rat} v_{s}(u):\left\langle(k, j), v_{s+1}(u)\right\rangle \geq 0\right\}
\end{gathered}
$$

for $0 \leq s \leq l-1$.

In [4] the vectors $(\rho, 1), v_{1}(u), \ldots, v_{l}(u)$ are called the invariants of $u$; clearly $v_{i}(u)$ is determined up to multiplication by a positive constant, and in the following we shall feel free to multiply this vector by any positive scalar.

Let us make some examples. If $u \in M_{\rho}^{r e c}$, with $\rho$ rational or irrational, it is easy to see that

$$
\Phi_{0}(u)=\{(k, j):\langle(k, j),(\rho, 1)\rangle \geq 0\}
$$

If $\rho$ is irrational, this comes from the fact that $: x_{n+1} \rightarrow U_{\rho}\left(x, x_{n+1}\right)$ is strictly monotone and $U_{\rho}\left(x, x_{n+1}\right)-x_{n+1}$ is periodic; if $\rho$ is rational, the formula above comes from a simple verification. Thus, if $u \in M_{\rho}^{r e c}, \Phi_{1}(u)=$ $\left(\mathbf{Z}^{n} \times \mathbf{Z}\right) \cap \operatorname{rat}(\rho, 1)$ and $l(u)=0$. The converse does not hold, if $\rho$ is irrational: as we shall see below, there can be $u \in M_{\rho}$ with $l(u)=0$ and not recurrent. Let now $n=1$ and $u \in M_{0}^{r e c}$, i.e. $u(x+1)=u(x)$; as we just said

$$
\Phi_{0}(u)=\{(k, j) \in \mathbf{Z} \times \mathbf{Z}: j \geq 0\}
$$

and $l(u)=0$. In the same setting, let $u_{1}<u_{2}$ be two elements of $M_{0}^{r e c}$ and let $u \in M_{0}$ satisfy

$$
\lim _{x \rightarrow-\infty}\left|u(x)-u_{1}(x)\right|=0=\lim _{x \rightarrow+\infty}\left|u(x)-u_{2}(x)\right| .
$$

Then

$$
\Phi_{0}(u)=\{(k, j): j>0\} \cup\{(k, j): j=0, k \geq 0\} .
$$

In this case, $l(u)=1$ and $v_{1}(u)=(1,0)$.

Let now $n \geq 2$, let $\rho=\left(\rho_{1}, \ldots, \rho_{s}, \rho_{s+1}, \ldots, \rho_{n}\right)$ with $\left(\rho_{1}, \ldots, \rho_{s}\right) \in \mathbf{Q}^{s}$ and $\left(\rho_{s+1}, \ldots, \rho_{n}\right)$ rationally independent. If $u \in M_{\rho}^{r e c}$, i.e. if $u(x)=u_{\rho}(x, \lambda)=U_{\rho}(x, \rho \cdot x+\lambda)$, we said before that

$$
\Phi_{0}(u)=\{(k, j): \rho \cdot k+j \geq 0\}
$$

Thus $\Phi_{1}(u)=\operatorname{rat}(\rho, 1) \cap\left(\mathbf{Z}^{n} \times \mathbf{Z}\right)$ and $l(u)=0$. But if $\lambda$ is a point of discontinuity of $: \lambda \rightarrow u_{\rho}(x, \lambda)$, if $u \in M_{\rho}$ satisfies

$$
u_{\rho}(x, \lambda-)<u(x)<u_{\rho}(x, \lambda+)
$$


and $u$ is periodic in the first $s$ coordinates, then $l=0$, but $u$ is not recurrent. More precisely, the graph of $u_{\rho}(x, \mu)$, when projected on $\mathbf{T}^{n+1}$ and closed, forms a Cantor set; this Cantor set does not depend on the parameter $\mu$ and $u$ lives in its gaps. There are examples of such solutions in [2,4]. Always if $\lambda$ is a point of discontinuity of $: \lambda \rightarrow u_{\rho}(x, \lambda)$, if $u \in M_{\rho}$ satisfies

$$
\lim _{t \rightarrow-\infty}\left|u\left(x+t w_{1}\right)-u_{\rho}\left(x+t w_{1}, \lambda-\right)\right|=0=\lim _{t \rightarrow+\infty}\left|u\left(x+t w_{1}\right)-u_{\rho}\left(x+t w_{1}, \lambda+\right)\right|
$$

uniformly for some $\left(w_{1},-\rho \cdot w_{1}\right) \in \operatorname{rat}(\rho, 1)$, then $l(u) \geq 1$ and $v_{1}(u)=\left(w_{1},-\rho \cdot w_{1}\right)$. If $\left(\rho_{1}, \ldots, \rho_{s}\right)=0$, if $u$ satisfies the formula above with $v_{1}(u)=e_{1}=(1,0, \ldots, 0)$ and is periodic in the directions $x_{2}, \ldots, x_{s}$, then $l(u)=1$. Let us suppose that there are two heteroclinics $\tilde{u}_{1}$ and $\tilde{u}_{2}$ satisfying the formula above with $v_{1}=e_{1}$; if $\bar{u} \in M_{\rho}$ is heteroclinic in the direction $e_{1}$ from $u_{\rho}(\cdot, \lambda-)$ to $u_{\rho}(\cdot, \lambda+)$, and in the direction $e_{2}=(0,1,0, \ldots, 0)$ from $\tilde{u}_{1}$ to $\tilde{u}_{2}$, then $l(u) \geq 2, v_{1}(u)=e_{1}$ and $v_{2}(u)=e_{2}$.

Definition. We say that $(\rho, 1), v_{1}, \ldots, v_{l}$ are admissible if $(2.1)$ holds. Since the $v_{i}$ are defined up to multiplication by a positive constant, we shall identify $(\rho, 1), v_{1}, \ldots, v_{l}$ with $(\rho, 1), w_{1}, \ldots, w_{l}$ if $v_{i}=\lambda_{i} w_{i}$ for some $\lambda_{i}>0$.

A theorem of [4] says the following: if $(\rho, 1), v_{1}, \ldots, v_{l}$ are admissible, then the set of all $u \in M_{\rho}$ such that $l(u) \leq l$ and $v_{i}(u)=v_{i}$ for $i \leq l(u)$, is ordered.

Definition. We define $M_{\left(\rho, v_{1}, \ldots, v_{s}\right)}$ as the set of all $u \in M_{\rho}$ which satisfy $v_{i}(u)=v_{i}$ for $i \leq \min (l(u), s)$.

We remark that this set in general is not ordered: for instance, if $l=0$ then it reduces to $M_{\rho}$ which in general is not ordered.

After these two definitions, it is natural to ask whether, given $(\rho, 1), v_{1}, \ldots, v_{l}$ admissible, there is $u \in M_{\rho}$ with $l(u)=l$ and $v_{i}(u)=v_{i}$ for $i \in(1, \ldots, l)$. In [4] it is proven that there is $u \in M_{\rho}$ with $l(u)=1$ and $v_{1}(u)=v_{1}$ iff the lamination of the $u \in M_{\rho}$ with $l(u)=0$ has gaps; there is $u \in M_{\rho}$ with $l(u)=2$ and $v_{1}(u)=v_{1}, v_{2}(u)=v_{2}$ iff the lamination of all the $u \in M_{\rho}$ with either $l(u)=0$ or $l(u)=1$ and $v_{1}(u)=v_{1}$ has gaps, and so on.

We want to understand the behaviour of $\Phi_{0}\left(u_{n}\right)$ when $u_{n} \rightarrow u$ in $C_{l o c}^{0}$. We list a few facts, easy to verify. 1) Let $A \subset B \subset \mathbf{Z}^{n} \times \mathbf{Z}$ be two semigroups such that $A \cup-A=B \cup-B=\mathbf{Z}^{n} \times \mathbf{Z}$; if $(\rho, 1), v_{1}, \ldots, v_{l}$ are the invariants of $A$, one checks easily that the invariants of $B$ are $(\rho, 1), v_{1}, \ldots, v_{l^{\prime}}$ with $l^{\prime} \leq l$.

2) $\Phi_{0}(u) \supset \liminf \Phi_{0}\left(u_{n}\right)$, i.e. $\Phi_{0}(u)$ contains those vectors which stay in $\Phi_{0}\left(u_{n}\right)$ from a certain $n$ onwards.

3) Possibly passing to a subsequence, we can suppose that $l\left(u_{n}\right)$ and $v_{i}\left(u_{n}\right)$ converge; the invariants of the semigroup liminf $\Phi_{0}\left(u_{n}\right)$ are the limits of the $v_{i}\left(u_{n}\right)$.

From the three properties above we get that $l(u) \leq \lim l\left(u_{n}\right)$ and $v_{i}(u)=\lim v_{i}\left(u_{n}\right)$ for $0 \leq i \leq l(u)$. A consequence of this is the following: let $u_{n} \rightarrow u$ and let $u_{n}\left(\cdot+k_{n}\right)+j_{n} \rightarrow w$ in $C_{l o c}^{0}\left(\mathbf{R}^{n}\right)$; then the set of the invariants of $u$ is contained in the set of the limits of the invariants of $\left\{u_{n}\right\}$, and the set of the invariants of $w$ is contained in the set of the limits of the invariants of $\left\{u_{n}\left(\cdot+k_{n}\right)+j_{n}\right\}$. But the invariants of $u_{n}$ are the same of $\left\{u_{n}\left(\cdot+k_{n}\right)+j_{n}\right\}$; thus the two sets of invariants of $u$ and $w$ respectively, are both contained in the same set; thus, either $l(u) \leq l(w)$ or $l(w) \leq l(u)$; in both cases, $v_{i}(u)=v_{i}(w)$ for $0 \leq i \leq \min (l(u), l(w))$.

We need to define the flats of $\alpha$. We recall that $\alpha$ is of class $C^{1}$ since $\beta$ is strictly convex and superlinear by [20]. Given $(\rho, 1) \in H_{n}\left(\mathbf{T}^{n+1}\right)$, the flat of slope $\rho$ is

$$
D_{\rho}=\left\{(c, \alpha(c)):-\alpha^{\prime}(c)=\rho\right\}
$$

Clearly, $D_{\rho} \subset H \times \mathbf{R}$ and it is a face of the hypograph of $\alpha$ with exterior normal $(\rho, 1)$. Given a convex set $D$, we denote by $D^{\circ}$ and by $\partial D$ the interior and the boundary of $D$ relative to the smallest affine subspace containing $D$; a face of $D$ is a flat of $\partial D$. By this definition, if $D$ is a point, then $D^{\circ}=D$ and $\partial D=\emptyset$. We define $F_{D}$ as the vector space generated by the differences $\left(c_{1}, \alpha\left(c_{1}\right)\right)-\left(c_{2}, \alpha\left(c_{2}\right)\right)$, with $\left(c_{i}, \alpha\left(c_{i}\right)\right) \in D$. We also recall that, if $D$ is convex and $v$ is a vector, then $v$ is an exterior normal to a unique face of $D$, namely the one on which

$$
\sup _{x \in D}\langle x, v\rangle
$$

is attained. 
Theorem 2.1. Let $\mathcal{L} \in C^{l, \gamma}$ with $l \geq 3$, and let $D_{\rho}$ be a flat of $\alpha$ of slope $\rho$; then the following holds.

(a) $F_{D_{\rho}} \subset \operatorname{rat}(\rho, 1)$; the dimension of $F_{D_{\rho}}$ is either the dimension of $\operatorname{rat}(\rho, 1)$ or zero; the latter happens iff $M_{\rho}$ is an ordered set.

(b) Let us suppose that $D_{\rho}$ is not zero-dimensional. Since $D_{\rho}$ is a convex set contained in $\operatorname{rat}(\rho, 1)$, each unit vector $v_{1} \in \operatorname{rat}(\rho, 1)$ is the exterior normal to a unique face of $D_{\rho}$, which we call $D_{\left(\rho, v_{1}\right)}$. We assert that the dimension of $D_{\left(\rho, v_{1}\right)}$ is either the dimension of $\operatorname{rat}(\rho, 1) \cap \operatorname{rat}\left(v_{1}\right)$ or zero; the latter happens iff $M_{\left(\rho, v_{1}\right)}$ is a lamination. Moreover, the correspondence face-normal is bijective.

(c) Let $\left(\rho, v_{1}, \ldots, v_{s-1}, v_{s}\right)$ be admissible; for $i \leq s$ we define iteratively $D_{\left(\rho, v_{1}, \ldots, v_{i}\right)}$ as the face of $D_{\left(\rho, v_{1}, \ldots, v_{i-1}\right)}$ with exterior normal $v_{i}$ when $D_{\left(\rho, v_{1}, \ldots, v_{i-1}\right)}$ is not reduced to a point; otherwise, we define it as the empty set. Then, if $D_{\left(\rho, v_{1}, \ldots, v_{i-1}\right)}$ is not reduced to a point, the correspondence between $v_{i}$ and the faces of $D_{\left(\rho, v_{1}, \ldots, v_{i-1}\right)}$ is bijective and

$$
F_{D_{\left(\rho, v_{1}, \ldots, v_{i}\right)}} \subset \operatorname{rat}(\rho, 1) \cap \operatorname{rat}\left(v_{1}\right) \cap \ldots \cap \operatorname{rat}\left(v_{i-1}\right) \cap \operatorname{rat}\left(v_{i}\right) .
$$

Moreover, the dimension of $D_{\left(\rho, v_{1}, \ldots, v_{i}\right)}$ is either the dimension of $\operatorname{rat}(\rho, 1) \cap \operatorname{rat}\left(v_{1}\right) \cap \ldots \cap \operatorname{rat}\left(v_{i-1}\right) \cap \operatorname{rat}\left(v_{i}\right)$ or zero, and the latter happens iff $M_{\left(\rho, v_{1}, \ldots, v_{i}\right)}$ is a lamination.

(d) $\partial D_{\left(\rho, v_{1}, \ldots, v_{s}\right)}$ is a $C^{1}$ surface in $\operatorname{rat}(\rho, 1) \cap \operatorname{rat}\left(v_{1}\right) \cap \ldots \cap \operatorname{rat}\left(v_{s}\right)$.

The directions of differentiability of a convex function are orthogonal to the corresponding flat of its polar; recalling this, point 1) of Theorem 2.1 has already been proven in [21].

Theorem 2.2 [21]. Let $(\rho, 1) \in H_{n}\left(\mathbf{T}^{n+1}\right)$ and let $S$ be the projection of $\operatorname{rat}(\rho, 1)^{\perp}$ on the first $n$ coordinates. Then $\beta$ restricted to $\rho+S$ is differentiable at $\rho$. Moreover, $\beta$ is differentiable at $\rho$ iff $M_{\rho}$ is a lamination.

To prove Theorem 2.1 we shall follow [13] and we shall study the relation between the flat of $\alpha$ of slope $\alpha^{\prime}(c)$ and the Aubry set at $c$.

\subsection{The Aubry sets and the flats of $\alpha$}

We recall from [16] the notion of the Aubry set in the 1-dimensional case. Let us suppose that, for any $\epsilon>0$, there is $u_{\epsilon}$, periodic of integer period $N_{\epsilon}$ (i.e. $\left.u_{\epsilon}\left(N_{\epsilon}\right)-u_{\epsilon}(0)=a_{\epsilon} \in \mathbf{Z}\right)$, such that

$$
0 \leq \int_{0}^{N_{\epsilon}}\left[\mathcal{L}\left(t, u_{\epsilon}, \dot{u}_{\epsilon}\right)-\eta_{c} \cdot \dot{u}_{\epsilon}-\alpha(c)\right] \mathrm{d} t \leq \epsilon
$$

where $\eta_{c}$ is a 1 -form representing $(c, 0) \in H^{1}\left(\mathbf{T}^{2}\right)$. It is proven in [16] that $u_{\epsilon}$ converges, up to subsequences in $C_{l o c}^{1}(\mathbf{R})$, to some $u \in M_{-\alpha^{\prime}(c)}$. The set of these limits is the Aubry set at $c$. From (2.2) we get that, if $T_{\epsilon}$ is a minimal current of rotation number $\left(\rho\left(u_{\epsilon}\right), 1\right)$, then

$$
0 \leq A\left(T_{\epsilon}\right)-T_{\epsilon}\left(\eta_{c}\right)-\alpha(c) \leq \frac{\epsilon}{N_{\epsilon}}
$$

where the inequality on the left comes from (1.19). We know that $\rho\left(u_{\epsilon}\right)=\frac{a_{\epsilon}}{N_{\epsilon}} \in \mathbf{Q}$; if $-\alpha^{\prime}(c)=\frac{p}{q}$ is rational too, and $\rho\left(u_{\epsilon}\right) \neq-\alpha^{\prime}(c)$, then it is easy to see that

$$
\frac{1}{q N_{\epsilon}} \leq\left|\rho\left(T_{\epsilon}\right)+\alpha^{\prime}(c)\right|
$$

and thus

$$
0 \leq A\left(T_{\epsilon}\right)-T_{\epsilon}\left(\eta_{c}\right)-\alpha(c) \leq q \epsilon\left|\rho\left(T_{\epsilon}\right)-\alpha^{\prime}(c)\right| .
$$

The formula above also holds when $\rho\left(u_{\epsilon}\right)=-\alpha^{\prime}(c)$, because in this case $A\left(T_{\epsilon}\right)-T_{\epsilon}\left(\eta_{c}\right)-\alpha(c)=0$ by (1.19). 
Also in the case when $-\alpha^{\prime}(c)$ is irrational, one can prove that, if $u_{\epsilon}$ is as in $(2.2)$ and $T_{\epsilon}$ is the current induced by a minimal $u$ with $\rho(u)=\rho\left(u_{\epsilon}\right)$, then

$$
0 \leq A\left(T_{\epsilon}\right)-T_{\epsilon}\left(\eta_{c}\right)-\alpha(c) \leq C \epsilon\left|\rho\left(T_{\epsilon}\right)-\alpha^{\prime}(c)\right|
$$

for some positive $C$. This is the property of the Aubry set we shall need in the following.

Definition. Let $-\alpha^{\prime}(c), v_{1}, \ldots, v_{s}$ be admissible. We say that $\mathcal{B} \subset M_{-\alpha^{\prime}(c)}$ is a $\left(-\alpha^{\prime}(c), v_{1}, \ldots, v_{s}\right)$-lamination at $c$ if $\mathcal{B}$ satisfies the following three conditions:

1) If $u \in \mathcal{B}$, then $l(u) \leq s$ and $v_{i}(u)=v_{i}$ for $i \leq l(u)$. In particular, by [4], $\mathcal{B}$ is an ordered set, a lamination.

2) $\mathcal{B}$ contains $u$ with invariants $\left(-\alpha^{\prime}(c), v_{1}, \ldots, v_{s}\right)$.

$3)$ Let $u$ has invariants $-\alpha^{\prime}(c), v_{1}, \ldots, v_{s^{\prime}}$ with $s^{\prime} \leq s$. Then $u \in \mathcal{B}$ iff there is $\left\{d_{i}\right\} \subset H$ and $u_{i} \in M_{-\alpha^{\prime}\left(d_{i}\right)}^{r e c}$ such that

and for any $\delta>0$ we eventually have

$$
u_{i} \rightarrow u \quad \text { in } \quad C_{l o c}^{1}\left(\mathbf{R}^{n}\right)
$$

$$
\begin{aligned}
\left|A\left(T_{i}\right)-T_{i}\left(\eta_{c}\right)-\alpha(c)\right| & =\left|\alpha\left(d_{i}\right)+\left\langle\alpha^{\prime}\left(d_{i}\right), c-d_{i}\right\rangle-\alpha(c)\right| \\
& \leq \delta\left\|\pi_{s^{\prime}-1}\left(\alpha^{\prime}\left(d_{i}\right)-\alpha^{\prime}(c)\right)\right\| .
\end{aligned}
$$

In the formula above, $\eta_{c}$ is a closed $n$-form representing $c \in H, T_{i}$ the current induced by $u_{i}$ and $\langle\cdot, \cdot\rangle$ the duality coupling between $H_{n}\left(\mathbf{T}^{n+1}\right)$ and $H^{n}\left(\mathbf{T}^{n+1}\right)$; we recall that, since $-\alpha$ is the polar of $\beta, \rho\left(T_{i}\right)=-\alpha^{\prime}\left(d_{i}\right)$ and thus $T_{i}\left(\eta_{c}\right)=\left\langle-\alpha^{\prime}\left(d_{i}\right), c\right\rangle$. To define the operators $\pi_{s}$, we consider $H_{n}\left(\mathbf{T}^{n+1}\right) \approx \mathbf{R}^{n+1}$, with the base defined in Section 1. We let $V_{-1}=\mathbf{R}^{n} \times\{0\}$ and we define $\pi_{-1}$ as the orthogonal projection on $V_{-1}$; we set

$$
V_{s}=\pi_{-1}\left[\operatorname{rat}\left(-\alpha^{\prime}(c), 1\right) \cap \operatorname{rat} v_{1} \cap \ldots \cap \operatorname{rat} v_{s}\right]
$$

and we let $\pi_{s}$ be the orthogonal projection on $V_{s}$.

We define $\mathcal{A}_{c}$, the Aubry set at $c$, as the union of all the $\left(-\alpha^{\prime}(c), v_{1}, \ldots, v_{s}\right)$-laminations at $c$.

We note that our definition is a little at variance with that of [16], where the Aubry set is the set of orbits approximated by some sequence $u_{\epsilon}$ satisfying (2.2); we ask, in addition, that these orbits are minimal and recurrent. In Lemma 2.12 we shall see that, when $n=1$, our definition coincides with that of [16].

The next lemma gives another interpretation of the Aubry set.

Lemma 2.3. Let $u \in M_{-\alpha^{\prime}(c)}$ have invariants $-\alpha^{\prime}(c), v_{1}, \ldots, v_{s}$, and let $V_{s} \subset H$ be defined as above. Let us suppose that

$$
d_{i} \rightarrow c, \quad d_{i}-c \in V_{s-1}
$$

or

$$
d_{i} \rightarrow c, \quad \alpha^{\prime}\left(d_{i}\right)-\alpha^{\prime}(c) \in V_{s-1} .
$$

Let $u_{i} \in M_{-\alpha^{\prime}\left(d_{i}\right)}^{r e c}$ and let $u_{i} \rightarrow u$ in $C_{l o c}^{1}\left(\mathbf{R}^{n}\right)$. Then $u$ belongs to a $\left(-\alpha^{\prime}(c), v_{1}, \ldots, v_{s}\right)$-lamination at $c$.

Proof. Let us suppose that (2.5) holds; since (2.3) holds by hypothesis, the lemma follows if we prove (2.4). We begin to note that, by $(2.5)$,

$$
\left|\left\langle\alpha^{\prime}\left(d_{i}\right)-\alpha^{\prime}(c), c-d_{i}\right\rangle\right|=\left|\left\langle\pi_{s-1}\left(\alpha^{\prime}\left(d_{i}\right)-\alpha^{\prime}(c)\right), c-d_{i}\right\rangle\right| \leq \frac{\delta}{2}\left\|\pi_{s-1}\left(\alpha^{\prime}\left(d_{i}\right)-\alpha^{\prime}(c)\right)\right\|
$$

for $i$ large; thus it suffices to prove that

$$
\left|\alpha\left(d_{i}\right)+\left\langle\alpha^{\prime}(c), c-d_{i}\right\rangle-\alpha(c)\right| \leq \frac{\delta}{2}\left\|\pi_{s-1}\left(\alpha^{\prime}\left(d_{i}\right)-\alpha^{\prime}(c)\right)\right\|
$$


for $i$ large. If we introduce the function

$$
g: V_{s-1} \rightarrow \mathbf{R}, \quad g(h)=\alpha(c+h)-\left\langle\alpha^{\prime}(c), h\right\rangle-\alpha(c)
$$

then (2.7) follows from

$$
|g(h)| \leq \frac{\delta}{2}\left\|g^{\prime}(h)\right\| \quad \text { if } \quad\|h\| \leq \epsilon(\delta)
$$

To prove this, we consider the concave function of one variable $f(t)=g(t h)$. We note that $t=0$ is a point of maximum of $f$, simply because $h=0$ is a point of maximum of $g$. The tangent to $f$ at the point $(t, f(t))$ intersects the ordinates at $y=f(t)-t f^{\prime}(t)$; since $f$ stays below its tangents and $f(0)=0$, we have $y \geq 0$ or equivalently $f(t) \geq t f^{\prime}(t)$. Since $t=0$ is a point of maximum of $f$, we have that $f(t) \leq 0$ and that $f^{\prime}(t) \leq 0$ if $t \geq 0$, while $f^{\prime}(t) \geq 0$ if $t \leq 0$. This and the last formula yields $|f(t)| \leq|t| \cdot\left|f^{\prime}(t)\right|$ for $t \in \mathbf{R}$. Going back to $g$, and setting $t h=k$, we get that

which implies $(2.8)$ for $\epsilon(\delta)=\frac{\delta}{2}$.

$$
|g(k)| \leq\left|\left\langle g^{\prime}(k), k\right\rangle\right|
$$

We now prove the lemma when (2.6) holds; first we note that, also in this case, the lemma follows if we prove (2.7). By the very same argument which yielded (2.8) we get that

$$
\left|\alpha\left(d_{i}\right)+\left\langle\alpha^{\prime}(c), c-d_{i}\right\rangle-\alpha(c)\right| \leq \frac{\delta}{2}\left\|\alpha^{\prime}\left(d_{i}\right)-\alpha^{\prime}(c)\right\|
$$

which implies (2.7) by (2.6).

The following proposition gives the relation between the Aubry set $\mathcal{A}_{c}$ and the flat of $\alpha$ containing $c$; essentially, the flat is normal to the invariants of the elements of $\mathcal{A}_{c}$.

Proposition 2.4. Let $D_{\rho}$ be a flat of $\alpha$ of slope $\rho$, and let $D$ denote a face of $D_{\rho}$, or a face of a face of $D_{\rho}$, etc... Then the following holds:

1) If $\left(c_{1}, \alpha\left(c_{1}\right)\right) \in D^{\circ},\left(c_{2}, \alpha\left(c_{2}\right)\right) \in D$, then $\mathcal{A}_{c_{1}} \subset \mathcal{A}_{c_{2}}$. In particular, if $\left(c_{1}, \alpha\left(c_{1}\right)\right),\left(c_{2}, \alpha\left(c_{2}\right)\right) \in D^{\circ}$, then $\mathcal{A}_{c_{1}}=\mathcal{A}_{c_{2}}$. Conversely, if $\left(c_{1}, \alpha\left(c_{1}\right)\right) \in D^{\circ}$ and $\mathcal{A}_{c_{1}} \subset \mathcal{A}_{c_{2}}$, then $\left(c_{2}, \alpha\left(c_{2}\right)\right) \in D$.

2) If $c \in D_{\left(\rho, v_{1}, \ldots, v_{s}\right)}^{\circ}$, then $\mathcal{A}_{c}$ is a $\left(\rho, v_{1}, \ldots, v_{s}\right)$-lamination.

3) We have that

$$
F_{D_{\left(\rho, v_{1}, \ldots, v_{s}\right)}} \subset \operatorname{rat}(\rho, 1) \cap \operatorname{rat} v_{1} \cap \ldots \cap \operatorname{rat} v_{s} .
$$

Moreover, the dimension of $F_{D_{\left(\rho, v_{1}, \ldots, v_{s}\right)}}$ is either the dimension of $\operatorname{rat}(\rho, 1) \cap \operatorname{rat} v_{1} \cap \ldots \cap$ rat $v_{i}$ or zero, and the latter happens iff $M_{\left(\rho, v_{1}, \ldots, v_{s}\right)}$ is a lamination.

Proof of Theorem 2.1. Point (a) follows from point 3) of Proposition 2.4; we shall not prove point (b) since it is a particular case of point (c). Point 3) of Proposition 2.4 implies almost all of point (c), except the assertion on the unique correspondence between $v_{s}$ and the faces of $D_{\left(\rho, v_{1}, \ldots, v_{s-1}\right)}$. To prove this, we begin to note that, if $D_{1}$ and $D_{2}$ are two different faces of $D_{\left(\rho, v_{1}, \ldots, v_{s-1}\right)}$, then they have different normals since $D_{\left(\rho, v_{1}, \ldots, v_{s-1}\right)}$ is convex. We want to prove the converse, i.e. that different normals correspond to different faces, or that $\partial D_{\left(\rho, v_{1}, \ldots, v_{s-1}\right)}$ has no corners. Let $c \in \partial D_{\left(\rho, v_{1}, \ldots, v_{s-1}\right)}$; we have that $c \in D^{\circ}$ with $D$ a face or subface of $D_{\left(\rho, v_{1}, \ldots, v_{s-1}\right)}$; we can write $D=D_{\left(\rho, v_{1}, \ldots, v_{s-1}, v_{s}, \ldots, v_{j}\right)}$. By point 2) of Proposition $2.4, \mathcal{A}_{c}$ is a $\left(\rho, v_{1}, \ldots, v_{s-1}, v_{s}, \ldots, v_{j}\right)$-lamination. Since $-\alpha^{\prime}(c), v_{1}, v_{2}, \ldots, v_{s-1}, v_{s}$ is admissible, we have that $v_{s}$ is orthogonal to $\left(-\alpha^{\prime}(c), 1\right), v_{1}, v_{2}, \ldots, v_{s-1} ;$ by the formula of point 3$)$, we get that $v_{s}$ is orthogonal to $\partial D_{\left(\rho, v_{1}, \ldots, v_{s-1}\right)}$ at $c$. Let us suppose by contradiction that $\partial D_{\left(\rho, v_{1}, \ldots, v_{s-1}\right)}$ has another normal at $c$, say $\tilde{v}_{s}$; by convexity, the face of $D_{\left(\rho, v_{1}, \ldots, v_{s-1}\right)}$ normal to $\tilde{v}_{s}$ is unique and contains $c$; by definition, this face is $D_{\left(\rho, v_{1}, \ldots, v_{s-1}, \tilde{v}_{s}\right)}$. Thus $c$ belongs to the interior both of $D_{\left(\rho, v_{1}, \ldots, v_{s-1}, v_{s}, \ldots, v_{j}\right)}$ and of $D_{\left(\rho, v_{1}, \ldots, v_{s-1}, \tilde{v}_{s}, \ldots, \tilde{v}_{k}\right)}$; but this contradicts point 2) of Proposition 2.4, because $\mathcal{A}_{c}$ should be contemporarily a $\left(\rho, v_{1}, \ldots, v_{s-1}, v_{s}, \ldots, v_{j}\right)$ and a $\left(\rho, v_{1}, \ldots, v_{s-1}, \tilde{v}_{s}, \ldots, \tilde{v}_{k}\right)$-lamination.

We have proven in the last paragraph that every point of $\partial D_{\left(\rho, v_{1}, \ldots, v_{s-1}\right)}$ has a unique normal; since $D_{\left(\rho, v_{1}, \ldots, v_{s-1}\right)}$ is convex, this implies that $\partial D_{\left(\rho, v_{1}, \ldots, v_{s-1}\right)}$ is of class $C^{1}$, i.e. point (d) of Theorem 2.1. 
We shall prove Proposition 2.4 in the following subsections.

\subsection{The Aubry set is constant in the interior of a face}

Lemma 2.5. Let $D_{\rho}$ be a flat of $\alpha$ of slope $\rho$, and let $D$ be a face of $D_{\rho}$, or a face of a face of $D_{\rho}$, etc. Then, if $\left(c_{0}, \alpha\left(c_{0}\right)\right) \in D^{\circ}$ and $\left(c_{1}, \alpha\left(c_{1}\right)\right) \in D$, we have that $\mathcal{A}_{c_{0}} \subset \mathcal{A}_{c_{1}}$. In particular, if also $\left(c_{1}, \alpha\left(c_{1}\right)\right) \in D^{\circ}$, then $\mathcal{A}_{c_{0}}=\mathcal{A}_{c_{1}}$.

Proof. If $\left(c_{0}, \alpha\left(c_{0}\right)\right) \in D^{\circ},\left(c_{1}, \alpha\left(c_{1}\right)\right) \in D$ and $\left(c_{0}, \alpha\left(c_{0}\right)\right) \neq\left(c_{1}, \alpha\left(c_{1}\right)\right)$, then we can find $\left(c_{2}, \alpha\left(c_{2}\right)\right) \in D$ and $\lambda \in(0,1)$ such that

$$
\left(c_{0}, \alpha\left(c_{0}\right)\right)=\lambda\left(c_{1}, \alpha\left(c_{1}\right)\right)+(1-\lambda)\left(c_{2}, \alpha\left(c_{2}\right)\right) .
$$

We have used the fact that $\alpha$ is affine on $D$. Let now $u$ belong to a $\left(-\alpha^{\prime}(c), v_{1}, \ldots, v_{s}\right)$-lamination at $c_{0}$; in other words, $u$ has invariants $-\alpha^{\prime}(c), v_{1}, \ldots, v_{s^{\prime}}$ with $s^{\prime} \leq s$ and there are $\left\{d_{i}\right\} \subset H$ and $u_{i} \in M_{-\alpha^{\prime}\left(d_{i}\right)}^{r e c}$ satisfying (2.3) and (2.4) at $c_{0}$. We want to prove that $u$ belongs to a $\left(-\alpha^{\prime}(c), v_{1}, \ldots, v_{s}\right)$-lamination at $c_{1}$, i.e. that $(2.4)$ holds at $c_{1}$ too. Let $T_{i}$ and $T$ be the currents induced by $u_{i}$ and $u$ respectively, and let $\eta_{c}$ be a $n$-form representing $c \in H$. We have that

$$
0 \leq A\left(T_{i}\right)-T_{i}\left(\eta_{c_{0}}\right)-\alpha\left(c_{0}\right) \leq \delta\left\|\pi_{s^{\prime}-1}\left(\alpha^{\prime}\left(d_{i}\right)-\alpha^{\prime}\left(c_{0}\right)\right)\right\|
$$

where the first inequality comes (1.19) and the second one is (2.4) at $c_{0}$. We get by (2.9) that

$$
A\left(T_{i}\right)-T_{i}\left(\eta_{c_{0}}\right)-\alpha\left(c_{0}\right)=\lambda\left[A\left(T_{i}\right)-T_{i}\left(\eta_{c_{1}}\right)-\alpha\left(c_{1}\right)\right]+(1-\lambda)\left[A\left(T_{i}\right)-T_{i}\left(\eta_{c_{2}}\right)-\alpha\left(c_{2}\right)\right]
$$

The equality above follows because $\left(c_{0}, \alpha\left(c_{0}\right)\right)$ and $\left(c_{1}, \alpha\left(c_{1}\right)\right)$ are on the same flat. Since both summands on the right are non-negative by (1.19), the last two formulas imply

$$
0 \leq A\left(T_{i}\right)-T_{i}\left(\eta_{c_{1}}\right)-\alpha\left(c_{1}\right) \leq \frac{\delta}{\lambda}\left\|\pi_{s^{\prime}-1}\left(\alpha^{\prime}\left(d_{i}\right)-\alpha^{\prime}\left(c_{0}\right)\right)\right\|=\frac{\delta}{\lambda}\left\|\pi_{s^{\prime}-1}\left(\alpha^{\prime}\left(d_{i}\right)-\alpha^{\prime}\left(c_{1}\right)\right)\right\|
$$

which proves (2.4) at $c_{1}$.

\subsection{The flat is contained in the rational space of the Aubry set}

Let $D_{\rho}$ be a flat of $\alpha$ of slope $\rho$, and let $D$ be a face of $D_{\rho}$, or a face of a face of $D_{\rho}$, etc. Let $F_{D}$ be the space generated by $D$. Our aim in this subsection is to prove that, if $c \in D^{\circ}$ and there is a $\left(-\alpha^{\prime}(c), v_{1}, \ldots, v_{s}\right)$ lamination at $c$, then

$$
F_{D} \subset \operatorname{rat}\left(-\alpha^{\prime}(c), 1\right) \cap \operatorname{rat} v_{1} \cap \ldots \cap \operatorname{rat} v_{s} .
$$

We shall do this in the following lemmas about the extension of closed $n$-forms; using ideas of [13], we shall prove that $F_{D}$ is contained in the space of closed forms which vanish on the $\left(-\alpha^{\prime}(c), v_{1}, \ldots, v_{s}\right)$-lamination; once we have this, the formula above follows easily. The heart of the matter Lemma 2.8 below on the extension of closed $n$-forms.

Let $c \in H$; we choose a $n$-form representing $c$; for simplicity, we choose the one with constant coefficients, which we still call $c$; we define

$$
f_{c}\left(x, x_{n+1}, p\right)=\mathcal{L}\left(x, x_{n+1}, p\right)-c \cdot p-\alpha(c) .
$$

We denote by $A_{c}$ the mean action of the Lagrangian $f_{c}$, and by $\beta_{c}$ and $\alpha_{c}$ the two conjugate functions. By the definitions of $\alpha$ and $\alpha_{c}$, it follows immediately that $\alpha_{c}(0)=0$ and that $\alpha_{c}(0)$ is attained on the same currents on which $\alpha(c)$ is attained; moreover, $\alpha^{\prime}(c)=\alpha_{c}^{\prime}(0)$ and thus $M_{-\alpha^{\prime}(c)}=M_{-\alpha_{c}^{\prime}(0)}$. In the following, we shall often switch from $\mathcal{L}$ to $f_{c}$, since many proofs are simpler if we suppose that $c=0$ and $\alpha(c)=0$. 
Lemma 2.6. Let $c \in H$, and let $f_{c}$ be as above. Let $-\alpha^{\prime}(c) \in \mathbf{Q}^{n}$ and let $\mathcal{B}=M_{-\alpha^{\prime}(c)}^{\text {rec }}$. Then there is a closed $n$-form $\eta_{\mathcal{B}, c}$ on $\mathbf{R}^{n+1}$ which is periodic in all its variables (i.e. it quotients on $\mathbf{T}^{n+1}$ ) and such that, $\forall u \in \mathcal{B}$ and $\forall x \in \mathbf{R}^{n}$,

$$
\left\{\begin{array}{l}
\eta_{\mathcal{B}, c}^{x}(x, u(x))=\partial_{p} f_{c}(x, u(x), \nabla u(x)) \\
\eta_{\mathcal{B}, c}^{u}(x, u(x))=f_{c}(x, u(x), \nabla u(x))-\left\langle\partial_{p} f_{c}(x, u(x), \nabla u(x)), \nabla u(x)\right\rangle .
\end{array}\right.
$$

In the formula above, $\eta_{\mathcal{B}, c}$ is expressed in the coordinates $\widehat{\mathrm{d} x_{i}}$ of Section 1 . The form $\eta_{\mathcal{B}, c}$ satisfies

$$
\left\|\eta_{\mathcal{B}, c}\left(x, x_{n+1}\right)-\eta_{\mathcal{B}, c}\left(z, z_{n+1}\right)\right\| \leq C\left\|\left(x, x_{n+1}\right)-\left(z, z_{n+1}\right)\right\|
$$

where the constant $C$ depends only on $\left\|\alpha^{\prime}(c)\right\|$. Moreover, if $\mathcal{B}$ is a foliation, $\eta_{\mathcal{B}, c}$ is exact and the flat of $\alpha$ at $c$ is reduced to a point.

Remark. We shall see in Lemma 2.8 below that, also if $\mathcal{B}$ is not a foliation, $\eta_{\mathcal{B}, c}$ can be modified outside $\mathcal{B}$ to an exact form.

Proof. The reference is obviously the part of [1] on foliations and calibrations; we shall proceed a little differently since we are not dealing with foliations, but with laminations; the problem is how to extend the form to the gaps of the lamination in such a way that (2.11) holds.

Let

$$
\Gamma=\left\{k \in \mathbf{Z}^{n}:-\alpha_{c}^{\prime}(0) \cdot k \in \mathbf{Z}\right\} .
$$

Since $-\alpha_{c}^{\prime}(0) \in \mathbf{Q}^{n}$, we have that $\Gamma$ is $n$-dimensional and we can find a bounded fundamental domain $A_{\Gamma}$ for the action of $\Gamma$ on $\mathbf{R}^{n}$.

We recall that $M_{-\alpha^{\prime}(c)}^{r e c}$ is an ordered set; let

$$
u_{0}<u_{1}<\ldots<u_{k}=u_{0}+1
$$

be elements of $M_{-\alpha^{\prime}(c)}^{r e c}$. We define the strip

$$
\tilde{M}_{i}=\left\{\left(x, x_{n+1}\right) \in \mathbf{R}^{n} \times \mathbf{R}: u_{i}(x)<x_{n+1}<u_{i+1}(x)\right\}
$$

and call $M_{i}$ its projection on $\mathbf{T}^{n+1}$. We shall need in the following that

$$
\left\{\left(x, x_{n+1}\right) \in A_{\Gamma} \times \mathbf{R}: u_{i}(x)<x_{n+1}<u_{i+1}(x)\right\}
$$

projects injectively on $\mathbf{T}^{n+1}$; by Lemma A1 of the Appendix, one sees that this is the case if

$$
u_{i+1}(x) \leq u_{i}(x+\bar{k})+\bar{j}
$$

where

$$
-\alpha^{\prime}(c) \cdot \bar{k}+\bar{j}=\min \left\{-\alpha^{\prime}(c) \cdot k+j>0:(k, j) \in \mathbf{Z}^{n} \times \mathbf{Z}\right\} .
$$

In the following, we shall always suppose that the $u_{i}$ satisfy the condition above. In particular, if $u_{i+1}<u_{i}+1$ then $\partial M_{i}$ has two components: the projection on $\mathbf{T}^{n+1}$ of $\left\{x_{n+1}=u_{j}(x), x \in A_{\Gamma}\right\}$ for $j=i, i+1$.

Step 1. We consider the vector field $\eta$, defined on $\partial M_{i}$ by (2.10); before extending it to a divergence-free vector field on $M_{i}$, we check that its flow across the boundary of $M_{i}$ is zero. Let $T_{i}$ be the current induced by $u_{i}$. We know that

$$
0=\alpha_{c}(0)=\min \left\{A_{c}(T): T \text { is induced by } u \in M_{\rho}, \rho \in \mathbf{R}^{n}\right\}=A_{c}\left(T_{i}\right)
$$

where the third equality comes from the remarks at the end of Section 1. Since

$$
A_{c}\left(T_{i}\right)=\frac{1}{\left|A_{\Gamma}\right|} \int_{A_{\Gamma}} f_{c}\left(x, u_{i}, \nabla u_{i}\right) \mathrm{d} x
$$


we have that

$$
\int_{A_{\Gamma}} f_{c}\left(x, u_{i}, \nabla u_{i}\right) \mathrm{d} x=0 .
$$

Since $\left.\eta\right|_{\partial M_{i}}$ is defined by (2.10), we get that

$$
\begin{aligned}
f_{c}\left(x, u_{i}, \nabla u_{i}\right)-\eta^{x}\left(x, u_{i}\right) \cdot \nabla & u_{i}-\eta^{u}\left(x, u_{i}\right)= \\
& f_{c}\left(x, u_{i}, \nabla u_{i}\right)-\left\langle\partial_{p} f_{c}\left(x, u_{i}, \nabla u_{i}\right), \nabla u_{i}\right\rangle-f_{c}\left(x, u_{i}, \nabla u_{i}\right)+\left\langle\partial_{p} f_{c}\left(x, u_{i}, \nabla u_{i}\right), \nabla u_{i}\right\rangle=0
\end{aligned}
$$

which implies

$$
\int_{A_{\Gamma}}\left[f_{c}\left(x, u_{i}, \nabla u_{i}\right)-\eta^{x}\left(x, u_{i}\right) \cdot \nabla u_{i}-\eta^{u}\left(x, u_{i}\right)\right] \mathrm{d} x=0 .
$$

From the last formula and (2.13) we get that

$$
\int_{A_{\Gamma}}\left[\eta^{x}\left(x, u_{i}\right) \cdot \nabla u_{i}+\eta^{u}\left(x, u_{i}\right)\right] \mathrm{d} x=0
$$

or equivalently

$$
\int_{\operatorname{Graph}\left(\left.u_{i}\right|_{A_{\Gamma}}\right)} \eta=0
$$

Step 2. By (2.14), the flow of $\eta$ across both sides of $M_{i}$ is zero; we want to extend $\eta$ to a divergence-free vector field (or closed $n$-form) $\eta_{i}$ on $M_{i}$. Moreover, we want

$$
\left\|\eta_{i}\left(x, x_{n+1}\right)-\eta_{i}\left(z, z_{n+1}\right)\right\| \leq C\left\|\left(x, x_{n+1}\right)-\left(z, z_{n+1}\right)\right\| .
$$

We want the constant $C$ to depend only on $\left\|\alpha^{\prime}(c)\right\|$, but not on the choice of the $\left\{u_{i}\right\}$.

We do this in the following way. We consider the strip

$$
\tilde{S}_{i}=\left\{\left(x, x_{n+1}\right): u_{i}(x) \leq x_{n+1} \leq u_{i}(x)+2\right\}
$$

which sits above the graph of $u_{i}$ and contains $\tilde{M}_{i}$. On $\tilde{S}_{i}$ we define a family of hypersurfaces, the graphs of

$$
u_{t}(x)=u_{i}(x)+t
$$

where $t \in[0,2]$. If we set

$$
g\left(x, u_{t}(x)\right)=\operatorname{div}_{p} f_{c}\left(x, u_{t}(x), \nabla u_{t}(x)\right)-\partial_{u} f_{c}\left(x, u_{t}(x), \nabla u_{t}(x)\right)
$$

we have that $u_{t}$ solves the elliptic problem

$$
\operatorname{div} \partial_{p} f_{c}\left(x, u_{t}(x), \nabla u_{t}(x)\right)=\partial_{u} f_{c}\left(x, u_{t}(x), \nabla u_{t}(x)\right)+g\left(x, u_{t}(x)\right) .
$$

We define

$$
G(x, s)=\int_{u_{i}(x)}^{s} g(x, \tau) \mathrm{d} \tau
$$

and we set $\tilde{f}_{c}(x, u, p)=f_{c}(x, u, p)+G(x, u)$. We have that $u_{t}$ satisfies

$$
\operatorname{div} \partial_{p} \tilde{f}_{c}\left(x, u_{t}(x), \nabla u_{t}(x)\right)=\partial_{u} \tilde{f}_{c}\left(x, u_{t}(x), \nabla u_{t}(x)\right) .
$$


Now the Lagrangian $\tilde{f}_{c}$ admits a foliation of solutions $u_{t}$; by the same calculations as in [1], this implies that the form $\eta_{i}$ which on $\left(x, u_{t}(x)\right) \in \tilde{S}_{i}$ takes value

$$
\left\{\begin{array}{l}
\eta_{i}^{x}\left(x, u_{t}(x)\right)=\partial_{p} \tilde{f}_{c}\left(x, u_{t}(x), \nabla u_{t}(x)\right) \\
\eta_{i}^{u}\left(x, u_{t}(x)\right)=\tilde{f}_{c}\left(x, u_{t}(x), \nabla u_{t}(x)\right)-\left\langle\partial_{p} \tilde{f}_{c}\left(x, u_{t}(x), \nabla u_{t}(x)\right), \nabla u_{t}(x)\right\rangle
\end{array}\right.
$$

is closed. Since $G=0$ on the graph of $u_{i}$, we have that $\eta_{i}$ satisfies $(2.10)$ on the graph of $u_{i}$. The problem is that we don't have $G=0$ on the graph of $u_{i+1}$, and thus $\eta_{i}$ does not satisfy $(2.10)$ on this set. To solve this, we let

$$
\tilde{S}_{i+1}=\left\{\left(x, x_{n+1}\right): u_{i+1}(x)-2 \leq x_{n+1} \leq u_{i+1}(x)\right\} .
$$

On $\tilde{S}_{i+1}$ we define a $n$-form $\eta_{i+1}$ as before; this form satisfies (2.10) on the graph of $u_{i+1}$. We note that, since

$$
u_{i+1}-2<u_{i}<u_{i-1}+2
$$

$\tilde{S}_{i}$ and $\tilde{S}_{i+1}$ contain $\tilde{M}_{i}$.

We now want to find a closed form $\bar{\eta}_{i}$ on $\tilde{M}_{i}$ which coincides with $\eta_{i+1}$ on the graph of $u_{i+1}$, and with $\eta_{i}$ on the graph of $u_{i}$; moreover, we want $\bar{\eta}_{i}$ periodic and satisfying (2.15). To do this, we define in $\tilde{M}_{i}$ two primitives of $\eta_{i}$ and $\eta_{i+1}$ by the Poincaré lemma ([10], Chap. 4). The two primitives are

$$
\gamma_{j}\left(x, x_{n+1}\right)\left(v_{1}, \ldots, v_{n-1}\right)=\int_{0}^{1} \beta_{j}\left(x, x_{n+1}, t\right)\left(\mathrm{d} i_{t}\left(v_{1}\right), \ldots, \mathrm{d} i_{t}\left(v_{n-1}\right)\right) \mathrm{d} t \quad j=i, i+1
$$

where

$$
i_{t}:\left(x, x_{n+1}\right) \rightarrow\left(x, x_{n+1}, t\right)
$$

and the $\beta_{j}$ are defined through the following homotopy

$$
H\left(x, x_{n+1}, t\right)=\left(x, u_{i+1}(x)+t\left(x_{n+1}-u_{i+1}(x)\right)\right) .
$$

Denoting by $H^{*}$ the pull-back by $H$, we set

$$
H^{*} \eta_{j}=\alpha+\mathrm{d} t \wedge \beta_{j}, \quad j=i, i+1
$$

where $\alpha$ does not contain terms in $\mathrm{d} t$. We have to derive a few estimates on $\beta_{j}$ and $\eta_{j}$.

Since the $u_{t}$ in $\tilde{S}_{i}$ are all translates of the same function, we have that

$$
\nabla u_{t}-\nabla u_{t^{\prime}}=0
$$

and the same holds for the $u_{t}$ in $\tilde{S}_{i+1}$ We now assert that

$$
\left\|\nabla u_{i+1}-\nabla u_{i}\right\|_{C^{0}\left(B\left(x_{0}, r\right)\right)} \leq C_{1}\left|u_{i+1}\left(x_{0}\right)-u_{i}\left(x_{0}\right)\right|
$$

with $C_{1}$ independent on $x_{0}$ and $r \leq 1$. To prove this, we recall from [17] that the map

$$
\Phi: \bigcup_{u \in M_{-\alpha^{\prime}(c)}^{r e c}} \operatorname{Graph}(u) \rightarrow \mathbf{R}^{n}, \quad \Phi:(x, u(x)) \rightarrow \nabla u(x)
$$

is Lipschitz; thus, (2.18) follows if we prove the Harnack-like inequality

$$
\left\|u_{i+1}-u_{i}\right\|_{C^{0}\left(B\left(x_{0}, r\right)\right)} \leq C_{2}\left|u_{i+1}\left(x_{0}\right)-u_{i}\left(x_{0}\right)\right|
$$


In other words, we have to show that

$$
\left|u_{i+1}(x)-u_{i}(x)\right| \leq C_{2}\left|u_{i+1}\left(x_{0}\right)-u_{i}\left(x_{0}\right)\right|
$$

for $\left\|x-x_{0}\right\| \leq r$. But this is a consequence of the Gronwall lemma, since

$$
\begin{array}{r}
\frac{\mathrm{d}}{\mathrm{d} t}\left[u_{i+1}\left(t x+(1-t) x_{0}\right)-u_{i}\left(t x+(1-t) x_{0}\right)\right]=\left\langle\nabla u_{i+1}\left(t x+(1-t) x_{0}\right)-\nabla u_{i}\left(t x+(1-t) x_{0}\right), x-x_{0}\right\rangle \\
\leq C_{3} r\left[u_{i+1}\left(t x+(1-t) x_{0}\right)-u_{i}\left(t x+(1-t) x_{0}\right)\right]
\end{array}
$$

where $C_{3}$ is the Lipschitz constant of $\Phi$.

By the definition of $\eta_{i},(2.18)$ and (2.19) imply that, if $r \leq 1$

$$
\left\|\left.\eta_{i+1}\right|_{\left(x, u_{i+1}(x)\right)}-\left.\eta_{i}\right|_{\left(x, u_{i}(x)\right)}\right\|_{C^{0}\left(B\left(x_{0}, r\right)\right)} \leq C_{4}\left|u_{i+1}\left(x_{0}\right)-u_{i}\left(x_{0}\right)\right| .
$$

For $r \leq \min \left(u_{i+1}\left(x_{0}\right)-u_{i}\left(x_{0}\right), 1\right)$, we define

$$
V_{x_{0}}=\left\{\left(x, x_{n+1}\right): x \in B\left(x_{0}, r\right), \quad u_{i}(x) \leq x_{n+1} \leq u_{i+1}(x)\right\} .
$$

Since $\mathcal{L} \in C^{l, \gamma}$ with $l \geq 3$, we can apply (3) with $l=3$; thus the first and second derivatives of $\nabla u_{j}, j=i, i+1$, are bounded; by the definition of $\eta_{j}$ and (2.17), the first and second derivatives of $\eta_{j}$ are bounded; by the last formula and (2.19), this implies that

$$
\left\{\begin{aligned}
\left\|\eta_{i+1}-\eta_{i}\right\|_{C^{0}\left(V_{x_{0}}\right)} & \leq C_{5}\left|u_{i+1}\left(x_{0}\right)-u_{i}\left(x_{0}\right)\right| \\
\left\|\eta_{i+1}\right\|_{C^{2}\left(V_{x_{0}}\right)}+\left\|\eta_{i}\right\|_{C^{2}\left(V_{x_{0}}\right)} & \leq C_{5}
\end{aligned}\right.
$$

with $C_{5}$ independent on $x_{0}$. By the last formula and (2.16), we get

$$
\left\{\begin{aligned}
\left\|\beta_{i+1}-\beta_{i}\right\|_{C^{0}\left(V_{x_{0}} \times[0,1]\right)} & \leq C_{6}\left|u_{i+1}\left(x_{0}\right)-u_{i}\left(x_{0}\right)\right| \\
\left\|\beta_{i+1}\right\|_{C^{2}\left(V_{x_{0}} \times[0,1]\right)}+\left\|\beta_{i}\right\|_{C^{2}\left(V_{x_{0}} \times[0,1]\right)} & \leq C_{6} .
\end{aligned}\right.
$$

If we choose $r \leq \min \left(u_{i+1}\left(x_{0}\right)-u_{i}\left(x_{0}\right), 1\right)$, we have that the diameter of $V_{x_{0}}$ is bounded by $\sqrt{n}\left|u_{i+1}\left(x_{0}\right)-u_{i}\left(x_{0}\right)\right|$; using this fact and integrating, the last formula yields

$$
\left\{\begin{aligned}
\left\|\gamma_{i+1}-\gamma_{i}\right\|_{C^{0}\left(V_{x_{0}}\right)} \leq C_{7}\left|u_{i+1}\left(x_{0}\right)-u_{i}\left(x_{0}\right)\right|^{2} \\
\left\|\gamma_{i+1}-\gamma_{i}\right\|_{C^{1}\left(V_{x_{0}}\right)} \leq C_{7}\left|u_{i+1}\left(x_{0}\right)-u_{i}\left(x_{0}\right)\right| \\
\left\|\gamma_{i+1}-\gamma_{i}\right\|_{C^{2}\left(V_{x_{0}}\right)} \leq C_{7}
\end{aligned}\right.
$$

with $C_{7}$ independent on $x_{0}$. We give a proof of the second one:

$$
\begin{aligned}
\left|\partial_{x_{s}}\left[\left(\gamma_{i+1}-\gamma_{i}\right)\left(v_{1}, \ldots, v_{n-1}\right)\right]\right| & \leq \int_{0}^{1} \mid \partial_{x_{s}}\left\{\left[\beta_{i+1}\left(x, x_{n+1}, t\right)-\beta_{i}\left(x, x_{n+1}, t\right)\right]\left(\mathrm{d} i_{t}\left(v_{1}\right), \ldots, \mathrm{d} i_{t}\left(v_{n-1}\right)\right\} \mid \mathrm{d} t\right. \\
& \leq C_{7}\left|u_{i+1}\left(x_{0}\right)-u_{i}\left(x_{0}\right)\right|
\end{aligned}
$$

where the first inequality comes from the definition of $\gamma_{i}, \gamma_{i+1}$; the second one comes from the fact that, by $(2.16)$,

$$
\begin{aligned}
& \left(\beta_{j+1}-\beta_{j}\right)\left(\mathrm{d} i_{t}\left(v_{1}\right), \ldots, \mathrm{d} i_{t}\left(v_{n-1}\right)\right)= \\
& \left.\sum_{k=1}^{n}(-1)^{k}\left[\eta_{j+1}-\eta_{j}\right]\right|_{H \circ i_{t}\left(x, x_{n+1}, t\right)}\left(\nabla_{x, x_{n+1}}\left(H \circ i_{t}\right)\left(v_{1}\right), \ldots, \nabla_{x, x_{n+1}}\left(H \circ i_{t}\right)\left(v_{k-1}\right), \partial_{t}\left(H \circ i_{t}\right)\left(v_{k}\right),\right. \\
& \left.\nabla_{x, x_{n+1}}\left(H \circ i_{t}\right)\left(v_{k+1}\right), \ldots, \nabla_{x, x_{n+1}}\left(H \circ i_{t}\right)\left(v_{n-1}\right)\right)
\end{aligned}
$$


and thus

$$
\begin{array}{r}
\int_{0}^{1}\left|\partial_{x_{s}}\left[\left(\beta_{j+1}-\beta_{j}\right)\left(\mathrm{d} i_{t}\left(v_{1}\right), \ldots, \mathrm{d} i_{t}\left(v_{n-1}\right)\right)\right]\right| \mathrm{d} t \leq \int_{0}^{1}\left|\sum_{k=1}^{n}(-1)^{k} \partial_{x_{s}}\left[\eta_{j+1}-\eta_{j}\right]\right|_{H \circ i_{t}\left(x, x_{n+1}, t\right)} \\
\times\left(\nabla_{x, x_{n+1}}\left(H \circ i_{t}\right)\left(v_{1}\right), \ldots, \nabla_{x, x_{n+1}}\left(H \circ i_{t}\right)\left(v_{k-1}\right), \partial_{t}\left(H \circ i_{t}\right)\left(v_{k}\right),\right. \\
\left.\nabla_{x, x_{n+1}}\left(H \circ i_{t}\right)\left(v_{k+1}\right), \ldots, \nabla_{x, x_{n+1}}\left(H \circ i_{t}\right)\left(v_{n-1}\right)\right)\left|\mathrm{d} t+\int_{0}^{1}\right| \sum_{s, k=1}^{n}\left(-1^{k}\right)\left[\eta_{j+1}-\eta_{j}\right] \\
\times\left(\nabla_{x, x_{n+1}}\left(H \circ i_{t}\right)\left(v_{1}\right), \ldots, \nabla_{x, x_{n+1}}\left(H \circ i_{t}\right)\left(v_{k-1}\right), \partial_{t}\left(H \circ i_{t}\right)\left(v_{k}\right), \nabla_{x, x_{n+1}}\left(H \circ i_{t}\right)\left(v_{k+1}\right), \ldots,\right. \\
\left.\nabla_{x, x_{n+1}}\left(H \circ i_{t}\right)\left(v_{k-1}\right), \partial_{x_{s}} \nabla_{x, x_{n+1}}\left(H \circ i_{t}\right)\left(v_{k}\right), \nabla_{x, x_{n+1}}\left(H \circ i_{t}\right)\left(v_{k+1}\right), \ldots, \nabla_{x, x_{n+1}}\left(H \circ i_{t}\right)\left(v_{n-1}\right)\right) \mid \mathrm{d} t \\
\quad \leq C_{8}\left|u_{i+1}\left(x_{0}\right)-u_{i}\left(x_{0}\right)\right| .
\end{array}
$$

The first integral above is estimated by $\frac{1}{2} C_{8}\left|u_{i+1}\left(x_{0}\right)-u_{i}\left(x_{0}\right)\right|$ because $\partial_{x_{s}}\left[\tilde{\eta}_{j+1}-\tilde{\eta}_{j}\right]$ and $\nabla_{x, x_{n+1}}\left(H \circ i_{t}\right)\left(v_{l}\right)$ are bounded, while

$$
\left\|\partial_{t}\left(H \circ i_{t}\right)\left(v_{k}\right)\right\| \leq C_{9}\left|u_{i+1}\left(x_{0}\right)-u_{i}\left(x_{0}\right)\right|
$$

where the last inequality comes from the estimate on the diameter of $V_{x_{0}}$. The second integral is smaller than $\frac{1}{2} C_{8}\left|u_{i+1}\left(x_{0}\right)-u_{i}\left(x_{0}\right)\right|$ by the first formula of $(2.20)$.

Now we consider

and we define on $M_{i}$

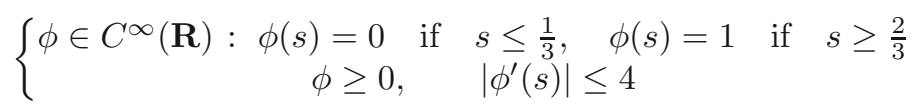

$$
\omega(x)=\left[1-\phi\left(\frac{x_{n+1}-u_{i}(x)}{u_{i+1}(x)-u_{i}(x)}\right)\right] \gamma_{i}\left(x, x_{n+1}\right)+\phi\left(\frac{x_{n+1}-u_{i}(x)}{u_{i+1}(x)-u_{i}(x)}\right) \gamma_{i+1}\left(x, x_{n+1}\right) .
$$

We set $\mathrm{d} \omega=\eta$ on $M_{i}$ and we see that $\mathrm{d} \omega$ is closed and that it satisfies $(2.10)$ on $\partial M_{i}$. Now

$$
\eta=[1-\phi] \eta_{i}+\phi \eta_{i+1}+\mathrm{d} \phi \wedge\left[\gamma_{i+1}-\gamma_{i}\right]
$$

We shall show that the derivatives of $\eta$ are bounded, i.e. that $\eta$ is Lipschitz. The norm of the derivatives $\partial_{x_{s}} \eta\left(x, x_{n+1}\right)$ contains the following terms:

$$
\begin{gathered}
\left|\partial_{x_{n+1}} \phi\right| \cdot\left\|\eta_{i+1}-\eta_{i}\right\|+[1-\phi] \cdot\left\|\partial_{x_{n+1}} \eta_{i}\right\|+\phi\left\|\partial_{x_{n+1}} \eta_{i+1}\right\| \\
\left\|\partial_{x_{n+1}} \mathrm{~d} \phi\right\| \cdot\left\|\gamma_{i+1}-\gamma_{i}\right\|+\|\mathrm{d} \phi\| \cdot\left\|\partial_{x_{n+1}}\left[\gamma_{i+1}-\gamma_{i}\right]\right\| \\
{[1-\phi] \cdot\left\|\nabla_{x} \eta_{i}\right\|+\phi\left\|\nabla_{x} \eta_{i+1}\right\|+\left\|\nabla_{x} \phi\right\| \cdot\left\|\eta_{i+1}-\eta_{i}\right\|} \\
\left\|\nabla_{x} \mathrm{~d} \phi\right\| \cdot\left\|\gamma_{i+1}-\gamma_{i}\right\|+\|\mathrm{d} \phi\| \cdot\left\|\nabla_{x}\left[\gamma_{i+1}-\gamma_{i}\right]\right\| .
\end{gathered}
$$

Since all these terms are bounded by $(2.20),(2.21)$ and $(2.22)$, we have that

$$
\left\|\eta\left(x, x_{n+1}\right)-\eta\left(z, z_{n+1}\right)\right\| \leq C_{13}\left\|\left(x, x_{n+1}\right)-\left(z, z_{n+1}\right)\right\| .
$$

The function $\eta$ just defined satisfies (2.10) on $M_{i}$.

On $\mathbf{T}^{n+1}$ we define the form $\tilde{\eta}$ by

$$
\tilde{\eta}\left(x, x_{n+1}\right)=\bar{\eta}_{i}\left(x, x_{n+1}\right) \quad \text { if } \quad\left(x, x_{n+1}\right) \in M_{i} \cup \partial M_{i} .
$$

Since $M_{i}$ and $M_{i+1}$ abut along the graph of $u_{i+1}$, where $\bar{\eta}_{i}=\bar{\eta}_{i+1}=\eta$ by $(2.10)$, we get by $(2.23)$ that $\tilde{\eta}$ satisfies (2.15). 
Now, either $M_{-\alpha^{\prime}(c)}^{r e c}$ is a finite set, or not. In the first case, we can suppose that the set of (2.12) coincides with $M_{-\alpha^{\prime}(c)}^{r e c}$, and then $\tilde{\eta}$ is a closed extension of $\eta$. In the second case, we can take finer and finer partitions in (2.12), so that, eventually, they approximate every element of $M_{-\alpha^{\prime}(c)}^{r e c}$; then we can pass to the limit using (2.15) and Ascoli-Arzelà.

Step 3. We suppose as in Steps 1 and 2 that $\alpha^{\prime}(c) \in \mathbf{Q}^{n}$; moreover, we suppose that $M_{-\alpha^{\prime}(c)}^{r e c}$ is a foliation. In this case, (2.10) defines defines a form $\eta$ on all $\mathbf{T}^{n+1}$; clearly, this form coincides with the $\tilde{\eta}$ defined in Step 2. We set $\eta_{\mathcal{B}, c}=\eta=\tilde{\eta}$; we want to prove that $\eta$ is exact.

Let $u \in M_{-\alpha^{\prime}(c)}^{r e c}$; since $\eta$ satisfies $(2.10)$, we have that

$$
\left.\partial_{p}\left[f_{c}(x, u(x), p)-\eta^{x}(x, u(x)) \cdot p-\eta^{u}(x, u(x))\right]\right|_{p=\nabla u(x)} \equiv 0
$$

Since $f_{c}-\eta^{x} \cdot p-\eta^{u}$ is convex in $p$, the last formula says that $p=\nabla u(x)$ is minimal along each fiber $(x, u(x)) \times \mathbf{R}^{n}$. From the second one of (2.10) it follows that the minimum is constantly zero:

$$
f_{c}(x, u(x), \nabla u(x))-\eta^{x}(x, u(x)) \cdot \nabla u(x)-\eta^{u}(x, u(x)) \equiv 0 .
$$

Since the $u \in M_{-\alpha^{\prime}(c)}^{r e c}$ form a foliation, we have that on each fiber $\left(x, x_{n+1}\right) \times \mathbf{R}^{n}$ the minimum is zero, which implies that

$$
f_{c}(x, u, p)-\eta^{x}(x, u) \cdot p-\eta^{u}(x, u) \geq 0 \quad \forall(x, u, p) .
$$

We have seen before that the elements of $M_{-\alpha^{\prime}(c)}^{\text {rec }}$ are minimal for $f_{c}$; from the last two formulas it follows that they are minimal also for $f_{c}-\eta$. Thus, $\alpha_{c}\left([\eta]^{x}\right)=A_{c}(T)-T\left(\eta^{x}\right)$ for a current $T$ induced by some $u \in M_{-\alpha^{\prime}(c)}^{r e c}$; taking the mean of (2.24) over a fundamental domain, we get that $[\eta]^{u}=\alpha_{c}\left([\eta]^{x}\right)$. It is a standard fact of Aubry-Mather theory that, if $\alpha_{c}(d)$ and $\alpha_{c}\left(d^{\prime}\right)$ are attained on the same current, then $\left(d, \alpha_{c}(d)\right)$ and $\left(d^{\prime}, \alpha_{c}\left(d^{\prime}\right)\right)$ lie on the same flat of $\alpha_{c}$; thus, we have that $(0,0)$ and $\left([\eta]^{x}, \alpha_{c}\left([\eta]^{x}\right)\right)$ lie on the same flat of $\alpha_{c}$. In particular, if we show that the flat at $[\eta]^{x}$ of $\alpha_{c}$ reduces to a point, we have that $[\eta]^{x}=0$. Since $[\eta]^{u}=\alpha_{c}\left([\eta]^{x}\right)$, we have that $[\eta]^{u}=\alpha_{c}(0)=0$; at the end $[\eta]=0$, which is what we wanted to show. Equivalently, we shall show that the flat of $\alpha$ at $c+[\eta]^{x}$ reduces to a point.

This means to show that, if $d^{x} \neq 0$, then

$$
\alpha\left(c+[\eta]^{x}+d^{x}\right)<\alpha\left(c+[\eta]^{x}\right)+\alpha^{\prime}\left(c+[\eta]^{x}\right) \cdot d^{x} .
$$

Let $[u]$ denote the homology class of $u \in M_{-\alpha^{\prime}(c)}^{r e c}$; we have seen in Section 1 that $[u]=\left(-\alpha^{\prime}(c), 1\right)$. In particular, given any $d^{x}$, we can always find $d^{u}$ such that $d=\left(d^{x}, d^{u}\right)$ is orthogonal to $[u]$; thus the formula above becomes:

$$
\text { if }\langle[u], d\rangle=0 \quad \text { and } \quad d \neq 0, \quad \text { then } \quad \alpha\left(c+[\eta]^{x}+d^{x}\right)<\alpha\left(c+[\eta]^{x}\right)+\alpha^{\prime}\left(c+[\eta]^{x}\right) \cdot d^{x}
$$

We want to write the formula above using the function $\tilde{\alpha}$ defined at the end of Section 1 . We recall that $\tilde{\alpha}\left(c+\lambda e_{n+1}\right)=\alpha(c)-\lambda$, so that $\tilde{\alpha}(c+[\eta]+d)=\alpha\left(c+[\eta]^{x}+d^{x}\right)-[\eta]^{u}-d^{u}$. Now $d \perp[u]=\left(-\alpha^{\prime}(c), 1\right) ;$ thus $d^{u}=\alpha^{\prime}(c) \cdot d^{x}$. Since we just saw that $c$ and $c+[\eta]^{x}$ are on the same flat, we have that $\alpha^{\prime}\left(c+[\eta]^{x}\right) \cdot d^{x}=$ $\alpha^{\prime}(c) \cdot d^{x}=d^{u}$. Moreover, we already know that $[\eta]^{u}=\alpha\left(c+[\eta]^{x}\right)$; thus the last formula becomes

$$
\text { if }\langle[u], d\rangle=0 \quad \text { and } \quad d \neq 0, \quad \text { then } \quad \tilde{\alpha}(c+[\eta]+d)<0
$$

To show (2.25), we let

and

$$
-\alpha^{\prime}(c)=\left(\frac{p_{1}}{q_{1}}, \ldots, \frac{p_{n}}{q_{n}}\right)
$$

$$
Q=\left\{x \in \mathbf{R}^{n}: 0 \leq x_{1}<q_{1}, \ldots, 0 \leq x_{n}<q_{n}\right\}
$$


Since $u$ repeats periodically outside $Q$, we get that the volume of $Q$ is a multiple of the volume of the fundamental domain of $u$. Since $\mathcal{B}$ is a foliation, we can choose the $\left\{u_{i}\right\}_{i=1}^{k}$ of $(2.12)$ in such a way that $u_{i}(0)=u_{0}(0)+\frac{i}{k}$. Now we fix a ball $B(0, r)$ such that $B(0, r) \supset Q ;(2.18)$ and $(2.19)$ hold for any fixed $r$ (actually we proved (2.19) only for $r \leq 1$, but it was only to have a constant independent on $r$ ), and since $u_{i}$ repeats periodically outside $Q$ we get

$$
\left\|u_{i+1}-u_{i}\right\|_{C^{1}\left(\mathbf{R}^{n}\right)} \leq \frac{C_{1}}{k}
$$

We define a function $\tilde{u}$ in the homology class of $k[u]+e_{1} \prod_{i=2}^{n} q_{i}$ in the following way. We set $\phi_{k}(t)=\phi(t \sqrt{k})$ where $\phi$ is the cutoff of $(2.22)$. We set

$$
\begin{aligned}
& \tilde{u}_{0}(x)=\left[1-\phi_{k}\left(x_{1}\right)\right]\left[u_{k-1}(x)-1\right]+\phi_{k}\left(x_{1}\right) u_{0}(x) \\
& \tilde{u}_{1}(x)=\left[1-\phi_{k}\left(x_{1}-q_{1}\right)\right] \tilde{u}_{0}(x)+\phi_{k}\left(x_{1}-q_{1}\right) u_{1}(x) \\
& \tilde{u}_{2}(x)=\left[1-\phi_{k}\left(x_{1}-2 q_{1}\right)\right] \tilde{u}_{1}(x)+\phi_{k}\left(x_{1}-2 q_{1}\right) u_{2}(x)
\end{aligned}
$$

up to

$$
\tilde{u}_{k}(x)=\left[1-\phi_{k}\left(x_{1}-k q_{1}\right)\right] \tilde{u}_{k-1}+\phi_{k}\left(x_{1}-k q_{1}\right) u_{k}(x)
$$

Now by $(2.12), u_{k}\left(x+k q_{1} e_{1}\right)=u_{0}(x)+k p_{1}+1$, which implies that, if $x_{1} \in\left[0, q_{1}\right]$, then $\tilde{u}_{k}\left(x+k q_{1} e_{1}\right)=\tilde{u}_{k}(x)+$ $k p_{1}+1$. From this it follows easily that we can find a continuous $\tilde{u}$ which coincides with $\tilde{u}_{k}$ on $\left[0, k q_{1}\right] \times \mathbf{R}^{n-1}$ and satisfies $\tilde{u}\left(x+k q_{1} e_{1}\right)=\tilde{u}(x)+k p_{1}+1$ for all $x$. Moreover, it is easy to see that the homology class of $\tilde{u}$, which we denote by $[\tilde{u}]$, satisfies $[\tilde{u}]=k[u]+e_{1} \prod_{i=2}^{n} q_{i}$ where $e_{i}$ is the basis defined in Section 1 .

Since (2.24) implies that

$$
\left.\int_{\left[i q_{1},(i+1) q_{1}\right] \times \prod_{j=2}^{n}\left[0, q_{j}\right]}\left[f_{c}\left(x, u_{i}, \nabla u_{i}\right)-\eta^{x}\left(x, u_{i}\right) \cdot \nabla u_{i}-\eta^{u}\left(x, u_{i}\right)\right]\right\} \mathrm{d} x=0
$$

we have that

$$
\begin{aligned}
& \int_{\left[0, k q_{1}\right] \times \prod_{j=2}^{n}\left[0, q_{j}\right]}\left[f_{c}(x, \tilde{u}, \nabla \tilde{u})-\eta^{x}(x, \tilde{u}) \cdot \nabla \tilde{u}-\eta^{u}(x, \tilde{u})\right] \mathrm{d} x= \\
& \sum_{i=0}^{k-1} \int_{\left[i q_{1},(i+1) q_{1}\right] \times \prod_{j=2}^{n}\left[0, q_{j}\right]}\left\{\left[f_{c}(x, \tilde{u}, \nabla \tilde{u})-\eta^{x}(x, \tilde{u}) \cdot \nabla \tilde{u}-\eta^{u}(x, \tilde{u})\right]\right. \\
&\left.-\left[f_{c}\left(x, u_{i}, \nabla u_{i}\right)-\eta^{x}\left(x, u_{i}\right) \cdot \nabla u_{i}-\eta^{u}\left(x, u_{i}\right)\right]\right\} \mathrm{d} x .
\end{aligned}
$$

Recalling that $u_{i}$ and $\tilde{u}$ coincide on $\left[i q_{1}+\frac{1}{\sqrt{k}},(i+1) q_{1}\right] \times \prod_{j=2}^{n}\left[0, q_{j}\right]$, we get that

$$
\begin{aligned}
& \int_{\left[0, k q_{1}\right] \times \prod_{j=2}^{n}\left[0, q_{j}\right]}\left[f_{c}(x, \tilde{u}, \nabla \tilde{u})-\eta^{x}(x, \tilde{u}) \cdot \nabla \tilde{u}-\eta^{u}(x, \tilde{u})\right] \mathrm{d} x= \\
& \sum_{i=0}^{k-1} \int_{\left[i q_{1}, i q_{1}+\frac{1}{\sqrt{k}}\right] \times \prod_{j=2}^{n}\left[0, q_{j}\right]}\left\{\left[f_{c}(x, \tilde{u}, \nabla \tilde{u})-\eta^{x}(x, \tilde{u}) \cdot \nabla \tilde{u}-\eta^{u}(x, \tilde{u})\right]\right. \\
&\left.-\left[f_{c}\left(x, u_{i}, \nabla u_{i}\right)-\eta^{x}\left(x, u_{i}\right) \cdot \nabla u_{i}-\eta^{u}\left(x, u_{i}\right)\right]\right\} \mathrm{d} x .
\end{aligned}
$$


U. BESSI

Setting $\tilde{u}=u_{i}+\psi_{i}$ on $\left[i q_{1}, i q_{1}+\frac{1}{\sqrt{k}}\right] \times \prod_{j=2}^{n}\left[0, q_{j}\right]$, we can write the last formula as

$$
\begin{gathered}
\int_{\left[0, k q_{1}\right] \times \prod_{j=2}^{n}\left[0, q_{j}\right]}\left[f_{c}(x, \tilde{u}, \nabla \tilde{u})-\eta^{x}(x, \tilde{u}) \cdot \nabla \tilde{u}-\eta^{u}(x, \tilde{u})\right] \mathrm{d} x= \\
\sum_{i=0}^{k-1} \int_{\left[i q_{1}, i q_{1}+\frac{1}{\sqrt{k}}\right] \times \prod_{j=2}^{n}\left[0, q_{j}\right]}\left\{\left[f_{c}\left(x, u_{i}, \nabla\left(u_{i}+\psi_{i}\right)\right)-\eta^{x}\left(x, u_{i}\right) \cdot \nabla\left(u_{i}+\psi_{i}\right)-\eta^{u}\left(x, u_{i}\right)\right]\right. \\
\left.-\left[f_{c}\left(x, u_{i}, \nabla u_{i}\right)-\eta^{x}\left(x, u_{i}\right) \cdot \nabla u_{i}-\eta^{u}\left(x, u_{i}\right)\right]\right\} \mathrm{d} x \\
+\sum_{i=0}^{k-1} \int_{\left[i q_{1}, i q_{1}+\frac{1}{\sqrt{k}}\right] \times \prod_{j=2}^{n}\left[0, q_{j}\right]} \begin{array}{r}
\left\{\left[f_{c}(x, \tilde{u}, \nabla \tilde{u})-\eta^{x}(x, \tilde{u}) \cdot \nabla \tilde{u}-\eta^{u}(x, \tilde{u})\right]\right. \\
-
\end{array} \\
\left.-\left[f_{c}\left(x, u_{i}, \nabla \tilde{u}\right)-\eta^{x}\left(x, u_{i}\right) \cdot \nabla \tilde{u}-\eta^{u}\left(x, u_{i}\right)\right]\right\} \mathrm{d} x .
\end{gathered}
$$

Since

we get by $(2.26)$ that

$$
\psi_{i}(x)=\left[1-\phi_{k}\left(x_{1}-i q_{1}\right)\right] \cdot\left[u_{i-1}(x)-u_{i}(x)\right]
$$

$$
\begin{cases}\left\|\psi_{i}\right\|_{C^{1}\left(\left[i q_{1}, i q_{1}+\frac{1}{\sqrt{k}}\right] \times \prod_{j=2}^{n}\left[0, q_{j}\right]\right)} & \leq \frac{C_{2}}{\sqrt{k}} \\ \left\|\psi_{i}\right\|_{C^{0}\left(\left[i q_{1}, i q_{1}+\frac{1}{\sqrt{k}}\right] \times \prod_{j=2}^{n}\left[0, q_{j}\right]\right)} & \leq \frac{C_{2}}{k}\end{cases}
$$

Since $\nabla u_{i}(x)$ minimizes $: p \rightarrow f_{c}\left(x, u_{i}(x), p\right)-\eta^{x}\left(x, u_{i}(x)\right) \cdot p-\eta^{u}\left(x, u_{i}(x)\right)$, this function has at most quadratic growth in a neighbourhood of $\nabla u_{i}(x)$ and thus

$$
\begin{aligned}
\|\left[f_{c}\left(x, u_{i}, \nabla\left(u_{i}+\psi_{i}\right)\right)-\right. & \left.\eta^{x}\left(x, u_{i}\right) \cdot \nabla\left(u_{i}+\psi_{i}\right)-\eta^{u}\left(x, u_{i}\right)\right] \\
& \quad-\left[f_{c}\left(x, u_{i}, \nabla u_{i}\right)-\eta^{x}\left(x, u_{i}\right) \cdot \nabla u_{i}-\eta^{u}\left(x, u_{i}\right)\right] \|_{L^{\infty}\left(\left[i q_{1}, i q_{1}+\frac{1}{\sqrt{k}}\right] \times \prod_{j=2}^{n}\left[0, q_{j}\right]\right)} \leq C_{3}\left(\frac{C_{2}}{\sqrt{k}}\right)^{2} .
\end{aligned}
$$

Using the fact that, by $(2.11),: z \rightarrow f_{c}(x, z, p)-\eta^{x}(x, p) \cdot p-\eta^{u}(x, z)$ is Lipschitz, we get

$\|\left[f_{c}(x, \tilde{u}, \nabla \tilde{u})-\eta^{x}(x, \tilde{u}) \cdot \nabla \tilde{u}-\eta^{u}(x, \tilde{u})\right]$

$$
-\left.\left[f_{c}\left(x, u_{i}, \nabla \tilde{u}\right)-\eta^{x}\left(x, u_{i}\right) \cdot \nabla \tilde{u}-\eta^{u}\left(x, u_{i}\right)\right]\right|_{L^{\infty}\left(\left[i q_{1}, i q_{1}+\frac{1}{\sqrt{k}}\right] \times \prod_{j=2}^{n}\left[0, q_{j}\right]\right)} \leq C_{3} \frac{C_{2}}{k} .
$$

From the last two formulas and (2.27) we get that

$$
\int_{\left[0, k q_{1}\right] \times \prod_{j=2}^{n}\left[0, q_{j}\right]}\left[f_{c}(x, \tilde{u}, \nabla \tilde{u})-\eta^{x}(x, \tilde{u}) \cdot \nabla \tilde{u}-\eta^{u}(x, \tilde{u})\right] \mathrm{d} x \leq \sum_{i=0}^{k-1} C_{3}\left[\left(\frac{C_{2}}{\sqrt{k}}\right)^{2}+\frac{C_{2}}{k}\right] \frac{1}{\sqrt{k}} \prod_{j=2}^{n} q_{j} \leq \frac{C_{4}}{\sqrt{k}} \prod_{j=2}^{n} q_{j} .
$$

Let now $d \in[u]^{\perp}$, and let $\eta_{d}$ be a $n$-form representing $d$; we have that

$$
\int_{\left[0, k q_{1}\right] \times \prod_{j=2}^{n}\left[0, q_{j}\right]}\left[\eta_{d}^{x} \cdot \nabla \tilde{u}+\eta_{d}^{u}\right] \mathrm{d} x=\int_{\operatorname{Graph}(\tilde{u})} \eta_{d}=\left\langle k[u]+e_{1} \prod_{j=2}^{n} q_{j}, d\right\rangle=\left\langle e_{1}, d\right\rangle \prod_{j=2}^{n} q_{j} .
$$

In the second equality, we have used the fact that $[\tilde{u}]=k[u]+e_{1} \prod_{i=2}^{n} q_{i}$, in the third we used the fact that $d \in[u]^{\perp}$. By the last two formulas we get that, if $\langle[u], d\rangle=0,\left\langle e_{1}, d\right\rangle>0$ and $k$ is sufficiently large,

$$
\int_{\left[0, k q_{1}\right] \times \prod_{j=2}^{n}\left[0, q_{j}\right]}\left[f_{c}(x, \tilde{u}, \nabla \tilde{u})-\eta^{x}(x, \tilde{u}) \cdot \nabla \tilde{u}-\eta^{u}(x, \tilde{u})-\eta_{d}^{x} \cdot \nabla \tilde{u}-\eta_{d}^{u}\right] \mathrm{d} x \leq \frac{C_{4}}{\sqrt{k}} \prod_{j=2}^{n} q_{j}-\left\langle e_{1}, d\right\rangle \prod_{j=2}^{n} q_{j}<0 .
$$


But this implies that $\tilde{\alpha}(c+[\eta]+d)<0$ if $d \in[u]^{\perp}$ and $\left\langle e_{1}, d\right\rangle>0$. Building a function $\tilde{u}$ as before, but in the homology class of $k[u]-e_{1} \Pi_{i=2}^{n} q_{i}$ (i.e. starting its construction from $u_{k}$ and not from $u_{1}$ ) one proves that $\tilde{\alpha}(c+[\eta]+d)<0$ also if $\left\langle d, e_{1}\right\rangle<0$. Applying this argument to the other coordinates we get that $(2.25)$ holds and we have seen that this implies that $\eta$ is exact.

We shall need the following extension of Step 3 of the last lemma.

Lemma 2.7. Let $f_{c}$ and $\alpha_{c}$ be as before, and let us suppose that

$$
-\alpha_{c}^{\prime}(0)=\left(\frac{p_{1}}{q_{1}}, \ldots, \frac{p_{n-1}}{q_{n-1}}, \frac{p_{n}}{q_{n}}\right)
$$

with $q_{n}$ relatively prime to $q_{i}, 1 \leq i \leq n-1$. Let $\mathcal{B}=M_{-\alpha^{\prime}(c)}^{\text {rec }}$, and let $\eta=\eta_{\mathcal{B}, c}$ be the $n$-form given by Lemma 2.6. Then there is $C>0$, only depending on $\left\|\alpha^{\prime}(c)\right\|$, such that

$$
\left|\left\langle e_{n},[\eta]\right\rangle\right| \leq \frac{C}{\sqrt{q}_{n}}
$$

More in general, let

$$
-\alpha_{c}^{\prime}(0)=\left(\frac{p_{1}}{q_{1}}, \ldots, \frac{p_{s}}{q_{s}}, \frac{p_{s+1}}{q_{s+1}}, \ldots \frac{p_{n}}{q_{n}}\right)
$$

and let us suppose that, for $i \in(s+1, \ldots, n), q_{i}$ is relatively prime to $q_{j}$ for $j \neq i$. Then

$$
\left|\left\langle e_{i},[\eta]\right\rangle\right| \leq \frac{C}{\sqrt{q_{i}}} \quad i \in(s+1, \ldots, n) .
$$

Proof. We begin to prove the first assertion. We shall proceed as in Step 3 of Lemma 2.6; since we shall build the test function $\tilde{u}$ gluing together several translates of $u \in M_{-\alpha_{c}^{\prime}(0)}^{r e c}$, we need some preliminaries on the fundamental domain of $-\alpha_{c}^{\prime}(0)$. We begin to consider

$$
\Gamma=\left\{k \in \mathbf{Z}^{n-1}:\left(\frac{p_{1}}{q_{1}}, \ldots, \frac{p_{n-1}}{q_{n-1}}\right) \cdot k \in \mathbf{Z}\right\}
$$

and a fundamental domain $A_{\Gamma} \subset \mathbf{R}^{n-1}$ of $\Gamma$.

Since $q_{n}$ is relatively prime with $q_{1}, \ldots, q_{n-1}$, Lemma A2 of the Appendix implies that $A_{\Gamma} \times\left[0, q_{n}\right]$ is a fundamental domain for $-\alpha^{\prime}(c)$. Let us now take $(k, j) \in \mathbf{Z}^{n} \times \mathbf{Z}$ such that $-\alpha^{\prime}(c) \cdot k+j>0$ is minimal; by Lemma A1 of the Appendix,

$$
\frac{1}{-\alpha^{\prime}(c) \cdot k+j}=l \in \mathbf{N} \quad \text { and } \quad u(x+l k)+l j=u(x)+1
$$

Let now $u \in M_{-\alpha^{\prime}(c)}^{r e c}$, and let

$$
B=\left\{\left(x, x_{n+1}\right): x \in A_{\Gamma} \times\left[0, q_{n}\right], u(x) \leq x_{n+1}<u(x+k)+j\right\} .
$$

By Lemma A1 of the Appendix, $B$ projects without self intersections onto $\mathbf{T}^{n+1}$ and $|B|=1$. But this is equivalent to say that

Clearly, this implies that

$$
\int_{A_{\Gamma} \times\left[0, q_{n}\right]}[u(x+k)+j-u(x)] \mathrm{d} x=1 .
$$

$$
\int_{A_{\Gamma} \times\left[0, q_{n}\right]}[u(x+s k)+s j-u(x+(s-1) k)-(s-1) j] \mathrm{d} x=1 \quad \forall s \in \mathbf{Z} .
$$


Now we have

$$
\begin{aligned}
1 & =\frac{1}{q_{n}\left|A_{\Gamma}\right|} \int_{A_{\Gamma} \times\left[0, q_{n}\right]}[u(x)+1-u(x)] \mathrm{d} x=\frac{1}{q_{n}\left|A_{\Gamma}\right|} \int_{A_{\Gamma} \times\left[0, q_{n}\right]}[u(x+l k)+l j-u(x)] \mathrm{d} x \\
& =\sum_{i=1}^{l} \frac{1}{q_{n}\left|A_{\Gamma}\right|} \int_{A_{\Gamma} \times\left[0, q_{n}\right]}\{u(x+i k)+i j-[u(x+(i-1) k)+(i-1) j]\} \mathrm{d} x=\frac{l}{q_{n}\left|A_{\Gamma}\right|}
\end{aligned}
$$

where the last equality comes from (2.29). From the last formula we get

$$
l=q_{n}\left|A_{\Gamma}\right|
$$

Moreover, from (2.29) with $s=1$ we get that there is $m \in\left[0, q_{n}-1\right] \cap \mathbf{Z}$ such that

$$
\int_{A_{\Gamma} \times[m, m+1]}[u(x+k)+j-u(x)] \mathrm{d} x \leq \frac{1}{q_{n}} .
$$

Translating, we can always suppose $m=0$. With the same argument, we have that

$$
\int_{A_{\Gamma} \times\left[0, \frac{1}{\sqrt{q_{n}}}\right]}[u(x+k)+j-u(x)] \mathrm{d} x \leq \frac{1}{q_{n} \sqrt{q_{n}}} .
$$

By (2.19), if $u\left(x_{0}+k\right)+j-u\left(x_{0}\right) \geq a>0$, then $u(x+k)+j-u(x) \geq \frac{a}{C}$ on a ball $B\left(x_{0}, 1\right)$, with $C$ not depending on $a$. This fact and (2.31) imply

$$
\|u(x+k)+j-u(x)\|_{C^{0}\left(\left\{0<x_{n}<1\right\}\right)} \leq \frac{C_{1}}{q_{n}}
$$

for some $C_{1}>0$. Since the map $\Phi:(x, u(x)) \rightarrow \nabla u(x)$ is Lipschitz, possibly enlarging $C_{1}$ we get

$$
\|\nabla[u(x+k)+j]-\nabla u(x)\|_{C^{0}\left(A_{\Gamma} \times[0,1]\right)} \leq \frac{C_{1}}{q_{n}} .
$$

Again using the fact that $\Phi:(x, u(x)) \rightarrow \nabla u(x)$ is Lipschitz, we get from (2.32) that

$$
\|\nabla[u(x+k)+j]-\nabla u(x)\|_{L^{1}\left(A_{\Gamma} \times\left[0, \frac{1}{\sqrt{q_{n}}}\right]\right)} \leq \frac{C_{1}}{q_{n} \sqrt{q_{n}}} .
$$

We now build a cycle in a way similar to Step 3 of Lemma 2.6. We let

$$
u_{i}(x)=u(x+i k)+i j, \quad \phi_{q_{n}}(t)=\phi\left(t \sqrt{q}_{n}\right)
$$

where $\phi$ is the cutoff of (2.22). We define

$$
\begin{aligned}
\tilde{u}_{0}(x) & =\left[1-\phi_{q_{n}}\left(x_{n}\right)\right] u_{-1}(x)+\phi_{q_{n}}\left(x_{n}\right) u_{0}(x) \\
\tilde{u}_{1}(x) & =\left[1-\phi_{q_{n}}\left(x_{n}-q_{n}\right)\right] \tilde{u}_{0}(x)+\phi_{q_{n}}\left(x_{n}-q_{n}\right) u_{1}(x) \\
\ldots & \\
\tilde{u}_{l}(x) & =\left[1-\phi_{q_{n}}\left(x_{n}-l q_{n}\right)\right] \tilde{u}_{l-1}(x)+\phi_{q_{n}}\left(x_{n}-l q_{n}\right) u_{l}(x) .
\end{aligned}
$$


As in Step 3 of Lemma 2.6, we can use the fact that $u_{l}(x)=u_{0}(x)+1$ to extend $\tilde{u}_{l}$ by periodicity outside $A_{\Gamma} \times\left[0, l q_{n}\right]$; we call the resulting function $\tilde{u}^{+}$. Again as in Step 3 of Lemma 2.6 we have that

$$
\begin{aligned}
& \int_{A_{\Gamma} \times\left[0, l q_{n}\right]}\left[f_{c}\left(x, \tilde{u}^{+}, \nabla \tilde{u}^{+}\right)-\eta^{x}\left(x, \tilde{u}^{+}\right) \cdot \nabla \tilde{u}^{+}-\eta^{u}\left(x, \tilde{u}^{+}\right)\right] \mathrm{d} x= \\
& \sum_{i=0}^{l-1} \int_{A_{\Gamma} \times\left[i q_{n},(i+1) q_{n}\right]}\left[f_{c}\left(x, \tilde{u}^{+}, \nabla \tilde{u}^{+}\right)-\eta^{x}\left(x, \tilde{u}^{+}\right) \cdot \nabla \tilde{u}^{+}-\eta^{u}\left(x, \tilde{u}^{+}\right)\right] \mathrm{d} x \\
& =\sum_{i=0}^{l-1} \int_{A_{\Gamma} \times\left[i q_{n},(i+1) q_{n}\right]}\left\{\left[f_{c}\left(x, \tilde{u}^{+}, \nabla \tilde{u}^{+}\right)-\eta^{x}\left(x, \tilde{u}^{+}\right) \cdot \nabla \tilde{u}^{+}-\eta^{u}\left(x, \tilde{u}^{+}\right)\right]\right. \\
& \left.-\left[f_{c}\left(x, u_{i}, \nabla u_{i}\right)-\eta^{x}\left(x, u_{i}\right) \cdot \nabla u_{i}-\eta^{u}\left(x, u_{i}\right)\right]\right\} \mathrm{d} x
\end{aligned}
$$

where the last equality comes from the fact that, by Step 1 of Lemma 2.6,

$$
\int_{A_{\Gamma} \times\left[i q_{n},(i+1) q_{n}\right]}\left[f_{c}\left(x, u_{i}, \nabla u_{i}\right)-\eta^{x}\left(x, u_{i}\right) \cdot \nabla u_{i}-\eta^{u}\left(x, u_{i}\right)\right] \mathrm{d} x=0 .
$$

Recalling that $u_{i}$ and $\tilde{u}^{+}$coincide on $A_{\Gamma} \times\left[i q_{n}+\frac{1}{\sqrt{q_{n}}}, i q_{n}\right]$, we get that

$$
\begin{aligned}
& \int_{A_{\Gamma} \times\left[0, l q_{n}\right]}\left[f_{c}\left(x, \tilde{u}^{+}, \nabla \tilde{u}^{+}\right)-\eta^{x}\left(x, \tilde{u}^{+}\right) \cdot \nabla \tilde{u}^{+}-\eta^{u}\left(x, \tilde{u}^{+}\right)\right] \mathrm{d} x= \\
& \sum_{i=0}^{l-1} \int_{A_{\Gamma} \times\left[i q_{n}, i q_{n}+\frac{1}{\sqrt{q_{n}}}\right]} \begin{array}{r}
\left\{\left[f_{c}\left(x, \tilde{u}^{+}, \nabla \tilde{u}^{+}\right)-\eta^{x}\left(x, \tilde{u}^{+}\right) \cdot \nabla \tilde{u}^{+}-\eta^{u}\left(x, \tilde{u}^{+}\right)\right]\right. \\
-
\end{array} \\
& \left.\quad\left[f_{c}\left(x, u_{i}, \nabla u_{i}\right)-\eta^{x}\left(x, u_{i}\right) \cdot \nabla u_{i}-\eta^{u}\left(x, u_{i}\right)\right]\right\} \mathrm{d} x .
\end{aligned}
$$

On $A_{\Gamma} \times\left[i q_{n}, i q_{n}+\frac{1}{\sqrt{q_{n}}}\right]$ we have that $\tilde{u}^{+}=u_{i}+\psi$, with

$$
\psi=\left[1-\phi_{q_{n}}\left(x_{n}-i q_{n}\right)\right] \cdot\left[u_{i-1}(x)-u_{i}(x)\right] .
$$

Taking derivatives and recalling (2.32), (2.33), (2.34) and (2.35), we get that

$$
\left\{\begin{aligned}
\|\nabla \psi\|_{L^{\infty}\left(A_{\Gamma} \times\left[i q_{n}, i q_{n}+\frac{1}{\sqrt{q_{n}}}\right]\right)} & \leq \frac{C_{1}}{\sqrt{q_{n}}} \\
\|\nabla \psi\|_{L^{1}\left(A_{\Gamma} \times\left[i q_{n}, i q_{n}+\frac{1}{\sqrt{q_{n}}}\right]\right)} & \leq \frac{C_{1}}{q_{n}} \\
\|\psi\|_{L^{1}\left(A_{\Gamma} \times\left[i q_{n}, i q_{n}+\frac{1}{\sqrt{q_{n}}}\right]\right)} & \leq \frac{C_{1}}{q_{n} \sqrt{q_{n}}} .
\end{aligned}\right.
$$

We now get that

$$
\begin{aligned}
\|\left[f_{c}\left(x, u_{i}, \nabla\left(u_{i}+\psi\right)\right)\right. & \left.-\eta^{x}\left(x, u_{i}\right) \cdot \nabla\left(u_{i}+\psi\right)-\eta^{u}\left(x, u_{i}\right)\right] \\
& -\left[f_{c}\left(x, u_{i}, \nabla u_{i}\right)-\eta^{x}\left(x, u_{i}\right) \cdot \nabla u_{i}-\eta^{u}\left(x, u_{i}\right)\right] \|_{L^{1}\left(A_{\Gamma} \times\left[i q_{n}, i q_{n}+\frac{1}{\sqrt{q_{n}}}\right]\right)} \\
\leq & C_{2} \int_{A_{\Gamma} \times\left[i q_{n}, i q_{n}+\frac{1}{\sqrt{q}_{n}}\right]}|\nabla \psi|^{2} \mathrm{~d} x \leq C_{2}\|\nabla \psi\|_{L^{\infty}} \int_{A_{\Gamma} \times\left[i q_{n}, i q_{n}+\frac{1}{\sqrt{\bar{q}_{n}}}\right]}|\nabla \psi| \mathrm{d} x \leq \frac{C_{3}}{q_{n} \sqrt{q_{n}}} .
\end{aligned}
$$

In the first inequality above we used the fact that $\nabla u_{i}(x)$ minimizes $: p \rightarrow f_{c}\left(x, u_{i}(x), p\right)-\eta^{x}\left(x, u_{i}(x)\right) \cdot p-$ $\eta^{u}\left(x, u_{i}(x)\right)$, in the last one we used the last formula. Analogously, since $: z \rightarrow f_{c}\left(x, z, \nabla \tilde{u}^{+}\right)-\eta^{x}(x, z) \cdot \nabla \tilde{u}^{+}-$ 
$\eta^{u}(x, z)$ is Lipschitz (with a Lipschitz constant depending only on $\left\|-\alpha^{\prime}(c)\right\|$ ), we get

$$
\begin{aligned}
& \|\left[f_{c}\left(x, \tilde{u}^{+}, \nabla \tilde{u}^{+}\right)-\eta^{x}\left(x, \tilde{u}^{+}\right) \cdot \nabla \tilde{u}^{+}-\eta^{u}\left(x, \tilde{u}^{+}\right)\right] \\
& -\left[f_{c}\left(x, u_{i}, \nabla \tilde{u}^{+}\right)-\eta^{x}\left(x, u_{i}\right) \cdot \nabla \tilde{u}^{+}-\eta^{u}\left(x, u_{i}\right)\right] \|_{L^{1}\left(A_{\Gamma} \times\left[i q_{n}, i q_{n}+\frac{1}{\bar{q}_{n}}\right]\right)} \leq \\
& C_{2}\|\psi\|_{L^{1}\left(A_{\Gamma} \times\left[i q_{n}, i q_{n}+\frac{1}{\sqrt{q}_{n}}\right]\right)} \leq \frac{C_{4}}{q_{n} \sqrt{q_{n}}} .
\end{aligned}
$$

Thus

$$
\begin{gathered}
\int_{A_{\Gamma} \times\left[i q_{n}, i q_{n}+\frac{1}{\left.\sqrt{q_{n}}\right]}\left\{\left[f_{c}\left(x, \tilde{u}^{+}, \nabla \tilde{u}^{+}\right)-\eta^{x}\left(x, \tilde{u}^{+}\right) \cdot \nabla \tilde{u}^{+}-\eta^{u}\left(x, \tilde{u}^{+}\right)\right]\right.\right.} \\
\left.-\left[f_{c}\left(x, u_{i}, \nabla u_{i}\right)-\eta^{x}\left(x, u_{i}\right) \cdot \nabla u_{i}-\eta^{u}\left(x, u_{i}\right)\right]\right\} \mathrm{d} x= \\
\int_{A_{\Gamma} \times\left[i q_{n}, i q_{n}+\frac{1}{\sqrt{q_{n}}}\right]}\left\{\left[f_{c}\left(x, u_{i}, \nabla\left(u_{i}+\psi\right)\right)-\eta^{x}\left(x, u_{i}\right) \cdot \nabla\left(u_{i}+\psi\right)-\eta^{u}\left(x, u_{i}\right)\right]\right. \\
+\int_{A_{\Gamma} \times\left[i q_{n}, i q_{n}+\frac{1}{\sqrt{q_{n}}}\right]}\left\{\left[f_{c}\left(x, \tilde{u}^{+}, \nabla \tilde{u}^{+}\right)-\eta^{x}\left(x, \tilde{u}^{+}\right) \cdot \nabla \tilde{u}^{+}-\eta^{u}\left(x, \tilde{u}^{+}\right)\right]\right. \\
\left.-\left[f_{c}\left(x, u_{i}, \nabla \tilde{u}^{+}\right)-\eta^{x}\left(x, u_{i}\right) \cdot \nabla \tilde{u}^{+}-\eta^{u}\left(x, u_{i}\right)\right]\right\} \mathrm{d} x \leq \frac{C_{3}}{q_{n} \sqrt{q_{n}}}+\frac{C_{4}}{q_{n} \sqrt{q_{n}}}=\frac{C_{5}}{q_{n} \sqrt{q_{n}}}
\end{gathered}
$$

where the inequality comes from (2.38) and (2.39). From the last formula and (2.37) we get

$$
\int_{A_{\Gamma} \times\left[0, l q_{n}\right]}\left[f_{c}\left(x, \tilde{u}^{+}, \nabla \tilde{u}^{+}\right)-\eta^{x}\left(x, \tilde{u}^{+}\right) \cdot \nabla \tilde{u}^{+}-\eta^{u}\left(x, \tilde{u}^{+}\right)\right] \mathrm{d} x \leq l \frac{C_{5}}{q_{n} \sqrt{q_{n}}}=\frac{C_{5}\left|A_{\Gamma}\right|}{\sqrt{q_{n}}}
$$

where the equality comes from (2.30). From the fact that $\left[\tilde{u}^{+}\right]=l\left[u_{i}\right]+e_{n}\left|A_{\Gamma}\right|$ we get that, if $d \in\left[u_{i}\right]^{\perp}$ and $\left\langle e_{n}, d\right\rangle>\frac{2 C_{5}}{\sqrt{q}_{n}}$, then

$$
\left\langle\left[\tilde{u}^{+}\right], d\right\rangle \geq \frac{2 C_{5}\left|A_{\Gamma}\right|}{\sqrt{q}_{n}}
$$

From this and (2.40), we get that, if $\eta_{d}$ is a $n$-form representing $d$, then

$$
\begin{aligned}
& \int_{A_{\Gamma} \times\left[0, l q_{n}\right]}\left[f_{c}\left(x, \tilde{u}^{+}, \nabla \tilde{u}^{+}\right)-\eta^{x}\left(x, \tilde{u}^{+}\right) \cdot \nabla \tilde{u}^{+}-\eta^{u}\left(x, \tilde{u}^{+}\right)-\eta_{d}^{x} \cdot \nabla \tilde{u}^{+}-\eta_{d}^{u}\right] \mathrm{d} x= \\
& \int_{A_{\Gamma} \times\left[0, l q_{n}\right]}\left[f_{c}\left(x, \tilde{u}^{+}, \nabla \tilde{u}^{+}\right)-\eta^{x}\left(x, \tilde{u}^{+}\right) \cdot \nabla \tilde{u}^{+}-\eta^{u}\left(x, \tilde{u}^{+}\right)\right] \mathrm{d} x-\left\langle\left[\tilde{u}^{+}\right], d\right\rangle \leq \frac{C_{5}\left|A_{\Gamma}\right|}{\sqrt{q}_{n}}-\frac{2 C_{5}\left|A_{\Gamma}\right|}{\sqrt{q}_{n}}<0 .
\end{aligned}
$$

We can build in the same way a periodic $\tilde{u}^{-}$with $\left[\tilde{u}^{-}\right]=l\left[u_{i}\right]-e_{n}\left|A_{\Gamma}\right|$ and such that

$$
\int_{A_{\Gamma} \times\left[0, m q_{n}\right]}\left[f_{c}\left(x, \tilde{u}^{-}, \nabla \tilde{u}^{-}\right)-\eta^{x}\left(x, \tilde{u}^{-}\right) \cdot \nabla \tilde{u}^{-}-\eta^{u}\left(x, \tilde{u}^{-}\right)-\eta_{d}^{x} \cdot \nabla \tilde{u}^{-}-\eta_{d}^{u}\right] \mathrm{d} x<0
$$

for $d \in\left[u_{i}\right]^{\perp}$ and $\left\langle e_{n}, d\right\rangle \leq \frac{-2 C_{5}}{\sqrt{q}_{n}}$. By (2.36) and the fact that $d \in\left[u_{i}\right]^{\perp}$ we get that

$$
\int_{A_{\Gamma} \times\left[i q_{n},(i+1) q_{n}\right]}\left[f_{c}\left(x, u_{i}, \nabla u_{i}\right)-\eta^{x}\left(x, u_{i}\right) \cdot \nabla u_{i}-\eta^{u}\left(x, u_{i}\right)-\eta_{d}^{x} \cdot \nabla u_{i}-\eta_{d}^{u}\right] \mathrm{d} x=0 .
$$


By the last three formulas, $u_{i}$ cannot minimize $f_{c}-\eta^{x} \cdot p-\eta^{u}-\eta_{d}^{x} \cdot p-\eta_{d}^{u}$ when $d \in\left[u_{i}\right]^{\perp}$ and $\left|\left\langle e_{n}, d\right\rangle\right| \geq \frac{2 C_{5}}{\sqrt{q}_{n}}$; since $u_{i}$ minimizes $f_{c}$, and the set of minimizers of $f_{c}-\eta-\eta_{d}$ depends only on the cohomology class of $\eta+\eta_{d}$, we have that the cohomology class of $\eta+\eta_{d}$ cannot be zero if $d \in\left[u_{i}\right]^{\perp}$ and $\left|\left\langle e_{n}, d\right\rangle\right| \geq \frac{2 C_{5}}{\sqrt{q}_{n}}$. Since we know by (2.14) that $\eta \in\left[u_{i}\right]^{\perp}$, we can take $\eta_{d}=-\eta$ and get that

$$
\left|[\eta]_{n}\right| \leq \frac{2 C_{5}}{\sqrt{q_{n}}}
$$

i.e. the thesis.

To prove the second assertion of the lemma, we notice that (2.28) is uniform in the first $n-1$ frequencies. We then apply this estimate separately to the $n$-th coordinate, to the $(n-1)$-th, all the way down to the $(s+1)$-th.

Remark. We refer the reader to Section 3 of [14] and to Theorem 3 of [21] for a more precise estimate on the width of the face containing $c$; see also [22] for a connection with the Miller indices of crystallographers.

Lemma 2.8. Let $c \in H$ and let $f_{c}$ be defined as before. Let $\mathcal{B}$ be $a\left(-\alpha^{\prime}(c), v_{1}, \ldots, v_{s}\right)$-lamination at $c$. Then there is an exact $n$-form $\eta_{(\mathcal{B}, c)}$ which satisfies $(2.10)$ on $\mathcal{B}$.

Proof. Let us begin with the case in which $\mathcal{B}$ is a $-\alpha^{\prime}(c)$-lamination.

We note that, if $u$ satisfies (1) and (2) for the Lagrangian $\mathcal{L}$ and $A$ is a unimodular transformation of $\mathbf{R}^{n}$, then $u(A x)$ satisfies $(1)$ and $(2)$ for $\mathcal{L}\left(A^{-1} x, u, p \cdot A\right)$. Since $\mathcal{L}\left(A^{-1} x, u, p \cdot A\right)$ satisfies the same hypotheses as $\mathcal{L}$, albeit with different constants, we can always consider $u(A x)$ instead of $u$. By Lemma A3 of the Appendix, we can choose $A$ in such a way that

$$
-\alpha^{\prime}(c)=\left(\rho_{1}, \ldots, \rho_{s}, \rho_{s+1}, \ldots, \rho_{n}\right)
$$

with $\left(\rho_{1}, \ldots, \rho_{s}\right) \in \mathbf{Q}^{s}$ and $\left(\rho_{s+1}, \ldots, \rho_{n}\right)$ rationally independent.

Thus we have to prove the following: if $\rho$ is as in (2.41) and if $c \in \pi_{-1}\left(D_{\rho}\right)$, there is a closed $n$-form $\eta_{c}$ such that

$$
\left\{\begin{array}{c}
\eta_{c} \text { satisfies }(2.10) \text { on } \mathcal{B} \text { for the Lagrangian } f_{c} \\
\eta_{c} \text { satisfies }(2.11) \\
{\left[\eta_{c}\right]=0 .}
\end{array}\right.
$$

Step 1. We note that $\pi_{-1}\left(D_{\rho}\right)$ is a convex set, since it is the projection on $\mathbf{R}^{n}$ of the convex $D_{\rho}$. Let $\tilde{D} \subset \pi_{-1}\left(D_{\rho}\right)$ be a set such that the closure of $\operatorname{co}(\tilde{D})$ is $\pi_{-1}\left(D_{\rho}\right)$. We assert that it is sufficient to find $\eta_{c}$ for $c \in \tilde{D}$.

First of all, it is easy to check that, if $\lambda \in[0,1]$ and if $\eta_{c_{1}}$ and $\eta_{c_{2}}$ satisfy (2.42) then also $(1-\lambda) \eta_{c_{1}}+\lambda \eta_{c_{2}}$ satisfies (2.42). Thus, we have (2.42) for all $c \in c o(\tilde{D})$. To extend (2.42) to the closure of $\operatorname{co}(\tilde{D})$, we consider $\left\{c_{i}\right\} \subset \operatorname{co}(\tilde{D})$ with $c_{i} \rightarrow c_{0}$; we let $\eta_{c_{i}}$ satisfy (2.42). Since $\eta_{c_{i}}$ satisfies (2.11), we can use Ascoli-Arzelà to get a subsequence $\eta_{c_{i^{\prime}}}$ converging uniformly to a $n$-form $\eta_{c_{0}}$. We want to prove that $\eta_{c_{0}}$ satisfies $(2.42)$. It is clear that $\eta_{c_{0}}$ satisfies (2.11); it also satisfies the last one of (2.42) since $\left[\eta_{c_{i}}\right]=0$ and the map : $\eta \rightarrow[\eta]$ is continuous for the uniform topology. We have to prove that $\eta_{c_{0}}$ satisfies $(2.10)$ for the Lagrangian $f_{c_{0}}$; but this follows easily from the fact that $\eta_{c_{i}}$ satisfies $(2.10)$ for $f_{c_{i}}$, and $f_{c}$ depends continuously on $c$.

Step 2. We define the set $\tilde{D}$ of Step 1.

Let $\pi_{-1}\left(D_{\rho}\right)$ be the projection of $D_{\rho}$ on $H$, and let $W_{\epsilon}$ be a $\epsilon$-neighbourhood of $\pi_{-1}\left(D_{\rho}\right)$ in $H$. Since $\alpha$ is concave, we get that, for any fixed $c_{0} \in \pi_{-1}\left(D_{\rho}\right)$,

$$
\left\langle\alpha^{\prime}(d)-\alpha^{\prime}\left(c_{0}\right), d-c_{0}\right\rangle<0 \quad \forall d \in \partial W_{\epsilon}
$$

This and the fact that $\alpha \in C^{1}$ imply by a standard argument of degree theory (see for instance [9], Th. 1.3.3) that $\alpha^{\prime}\left(W_{\epsilon}\right)$ contains a ball centered in $\alpha^{\prime}\left(c_{0}\right)$. Let now $v$ be an exterior normal to $\pi_{-1}\left(D_{\rho}\right)$ at $\bar{c} \in \pi_{-1}\left(D_{\rho}\right)$; 
in other words, $v$ is the slope of any plane $: c \rightarrow\langle v, c\rangle+a$ such that $\langle v, c\rangle+a \leq 0$ for $c \in \pi_{-1}\left(D_{\rho}\right)$ and $\langle v, \bar{c}\rangle+a=0$. Since $\alpha^{\prime}\left(V_{\epsilon}\right) \supset B\left(\alpha^{\prime}\left(c_{0}\right), r\right)$, we can find $d_{k} \in H$ such that

$$
\left\{\begin{array}{c}
-\alpha^{\prime}\left(d_{k}\right)=\left(\frac{p_{k}^{1}}{q_{k}^{1}}, \ldots, \frac{p_{k}^{n}}{q_{k}^{n}}\right) \in \mathbf{Q}^{n} \\
q_{k}^{i} \text { and } q_{k}^{j} \text { are relatively prime if } i \neq j \\
\alpha^{\prime}\left(d_{k}\right) \rightarrow \alpha^{\prime}\left(c_{0}\right) \\
\frac{\alpha^{\prime}\left(d_{k}\right)-\alpha^{\prime}\left(c_{0}\right)}{\left\|\alpha^{\prime}\left(d_{k}\right)-\alpha^{\prime}\left(c_{0}\right)\right\|} \rightarrow-v \\
q_{k}^{j} \rightarrow+\infty \text { for } j=1, \ldots, n .
\end{array}\right.
$$

Since $-\alpha$ is convex and superlinear, the third one of (2.43) implies that, up to a subsequence, $d_{k} \rightarrow d \in \pi_{-1}\left(D_{\rho}\right)$. Again by concavity, we have that

$$
\left\langle\alpha^{\prime}\left(d_{k}\right)-\alpha^{\prime}(\bar{c}), d_{k}-\bar{c}\right\rangle \leq 0 .
$$

By the fourth one of (2.43) we deduce that

$$
\langle v, d-\bar{c}\rangle \geq 0
$$

which implies that $d$ may be different from $\bar{c}$, but lies on the same face of $\pi_{-1}\left(D_{\rho}\right): v$ is an exterior normal both at $\bar{c}$ and at $d$.

We define $\tilde{D}$ as the set of all points $d$ obtained as above as $v$ varies in $H$. We want to prove that the closure of $c o(\tilde{D})$ equals $\pi_{-1}(D)$.

Let us suppose that the closure of $c o(\tilde{D})$ is strictly contained in $\pi_{-1}\left(D_{\rho}\right)$; then there is a plane $: c \rightarrow\langle v, c\rangle+a$ such that $\langle v, c\rangle+a \leq 0$ for $c$ in the convex hull, and $\langle v, \tilde{c}\rangle+a>0$ for some $\tilde{c} \in \pi_{-1}\left(D_{\rho}\right)$. For this $v$, we take a sequence $d_{k}$ as in (2.43) and we call $d$ its limit; using the concavity of $\alpha$, it is easy to see that $\langle v, d-\tilde{c}\rangle \geq 0$. Since $\langle v, \tilde{c}\rangle+a>0$, we get that $\left\{d_{k}\right\}$ converges to a point $d \in \tilde{D}$ with $\langle v, d\rangle+a>0$, a contradiction.

Step 3. We prove that $(2.42)$ holds for $c \in \tilde{D}$.

Let $d_{k}$ be as in (2.43), and let $c$ be its limit; let $\mathcal{B}_{k}=M_{-\alpha^{\prime}\left(d_{k}\right)}^{r e c}$ and let us consider $\eta_{\mathcal{B}_{k}, d_{k}}$ given by Lemma 2.6. By (2.11) and Ascoli-Arzelà we can suppose that $\eta_{\mathcal{B}_{k}, d_{k}}$ converges in $C_{l o c}^{0}$ to a $n$-form $\eta_{c}$. By (2.14) we have that

$$
\left[\eta_{\mathcal{B}_{k}, d_{k}}\right] \in\left(-\alpha^{\prime}\left(d_{k}\right), 1\right)^{\perp}
$$

and since $-\alpha^{\prime}\left(d_{k}\right) \rightarrow-\alpha^{\prime}(c)$, we get

We want to apply Lemma 2.7 to

$$
\left[\eta_{c}\right] \in\left(-\alpha^{\prime}(c), 1\right)^{\perp}
$$

$$
-\alpha^{\prime}\left(d_{k}\right)=\left(\rho_{1}^{k}, \ldots, \rho_{s}^{k}, \rho_{s+1}^{k}, \ldots, \rho_{n}^{k}\right) .
$$

By the fifth one of (2.43), the denominators of $\rho_{1}^{k}, \ldots, \rho_{n}^{k}$ tend to $+\infty$; moreover, by the second one of (2.43) each denominator is relatively prime to the denominators of all the other $\rho_{j}^{k}$. Thus Lemma 2.7 applies and we get

$$
\lim _{k \rightarrow+\infty}\left[\eta_{\mathcal{B}_{k}, d_{k}}\right]_{j}=0 \quad \text { if } \quad j=1, \ldots, n .
$$

Since $\eta$ is the limit of $\eta_{\mathcal{B}_{k}, d_{k}}$, we have that

$$
\left[\eta_{c}\right]_{j}=0 \quad \text { if } \quad j=1, \ldots, n .
$$

Thus $\eta_{c}$ satisfies the third one of (2.42); the second one of (2.42) follows easily. We have to show that $\eta_{c}$ satisfies (2.10) on $\mathcal{B}$. By (3) and Ascoli-Arzelà, the elements of $M_{-\alpha^{\prime}\left(d_{k}\right)}^{r e c}$ converge to the elements of a lamination $\mathcal{C} \subset M_{-\alpha^{\prime}(c)}$; moreover, $\eta_{c}$ clearly satisfies $(2.10)$ on $\mathcal{C}$. We also note that the elements of $M_{-\alpha^{\prime}\left(d_{k}\right)}^{r e c}$ intersect the elements of $\mathcal{B}$, since by $(2.43)-\alpha^{\prime}\left(d_{i}\right) \neq-\alpha^{\prime}(c)$. Now by the same arguments as in the proof of Lemma 2.12 below we get that $\mathcal{B} \subset \mathcal{C}$, i.e., $\eta$ satisfies $(2.10)$ on $\mathcal{B}$. 
Step 4. We now define a form $\eta$ satisfying $(2.42)$ when $\mathcal{B}$ is a $\left(-\alpha^{\prime}(c), v_{1}\right)$-lamination at $c$. By Lemma A3 in the Appendix, we can suppose that, setting as usual $v_{1}=\left(v_{1}^{x}, v_{1}^{u}\right)$,

$$
v_{1}^{x}=\left(\nu_{1}, \ldots, \nu_{s^{\prime}}, \nu_{s^{\prime}+1}, \ldots, \nu_{s}, 0, \ldots, 0\right)
$$

with $\left(\nu_{1}, \ldots, \nu_{s^{\prime}}\right) \in \mathbf{Q}^{s^{\prime}}$ and $\left(\nu_{s^{\prime}+1}, \ldots, \nu_{s}\right)$ rationally independent.

By Step 3, we know that, setting $\mathcal{C}=M_{-\alpha^{\prime}(c)}^{r e c}$, we can find an exact form $\eta_{\mathcal{C}, c}$ which satisfies $(2.10)$ on $M_{-\alpha^{\prime}(c)}^{r e c}$; applying Lemmas 2.9 and 2.10 below to this situation, we get that the space generated by $D_{\rho}$ is contained in $\operatorname{rat}(\rho, 1)$. On the other hand, since $\left(\rho, v_{1}\right)$ is admissible, we have that $v_{1} \in \operatorname{rat}(\rho, 1)$. Since $\mathcal{B}$ is a $\left(-\alpha^{\prime}(c), v_{1}\right)-$ lamination, we can find $\left\{d_{i}\right\}$ and $u_{i} \in M_{-\alpha^{\prime}\left(d_{i}\right)}^{r e c}$ satisfying (2.3) and (2.4) at $c$. Let

$$
\Gamma=\left\{(k, j) \in \mathbf{Z}^{n} \times \mathbf{Z}:-\alpha^{\prime}(c) \cdot k+j=0\right\} .
$$

Since $u_{i}$ converges to a solution with invariants $-\alpha^{\prime}(c), v_{1}$, we must have

$$
\liminf \left\{(k, j) \in \Gamma: u_{i}(x+k+j)>u_{i}(x)\right\} \subset \Gamma \cap\left\{\left\langle(k, j), v_{1}\right\rangle \geq 0\right\}
$$

or equivalently

$$
\liminf \left\{(k, j) \in \Gamma:-\alpha^{\prime}\left(d_{i}\right) \cdot k+j>0\right\} \subset \Gamma \cap\left\{\left\langle(k, j), v_{1}\right\rangle \geq 0\right\} .
$$

Since $-\alpha^{\prime}(c) \cdot k+j=0$ for $(k, j) \in \Gamma$ and $v_{1} \in \operatorname{rat}(\rho, 1)$, the formula above implies that

$$
\begin{aligned}
& \liminf \left\{(k, j) \in \Gamma:-\alpha^{\prime}\left(d_{i}\right) \cdot k+j>0\right\} \subset\{(k, j) \in \Gamma:\left.\left\langle(k, j), v_{1}\right\rangle \geq 0\right\}= \\
&\left\{\left(k, \alpha^{\prime}(c) \cdot k\right) \in \Gamma: k \cdot v_{1}^{x}+\left(\alpha^{\prime}(c) \cdot k\right)\left(\alpha^{\prime}(c) \cdot v_{1}^{x}\right) \geq 0\right\}
\end{aligned}
$$

The last formula implies that, for some $\lambda>0$,

$$
\frac{-\alpha^{\prime}\left(d_{i}\right)+\alpha^{\prime}(c)}{\left\|-\alpha^{\prime}\left(d_{i}\right)+\alpha^{\prime}(c)\right\|} \rightarrow v:=\lambda\left[v_{1}^{x}+\left(\alpha^{\prime}(c) \cdot v_{1}^{x}\right) \alpha^{\prime}(c)\right]
$$

and

$$
v \in \pi_{-1}\left(\operatorname{rat}\left(-\alpha^{\prime}(c), 1\right)\right)=V_{0}
$$

where the space $V_{0}$ is the same as in the definition of a $\left(-\alpha^{\prime}(c), v_{1}\right)$-lamination. An easy calculation that the vector $v$ is an exterior normal to the face $\pi_{-1}\left(D_{\rho, v_{1}}\right)$ of $\pi_{-1}\left(D_{\rho}\right)$.

By the formula above and the concavity argument we already employed in Step 2, we have that, if $u_{i} \in$ $M_{-\alpha^{\prime}\left(d_{i}\right)}^{r e c}$ converges to a $\left(-\alpha^{\prime}(c), v_{1}\right)$-lamination at $c$, then $d_{i}$ converges to the face of $\pi_{-1}\left(D_{\rho}\right)$ with exterior normal $v$. Let us call this face $M$. We want to proceed as in Steps 1 and 2: we want to find a set $\tilde{M} \subset M$ whose closed convex hull is $M$, and such that there is an exact form $\eta_{(\mathcal{B}, d)}$ satisfying $(2.10)$ and $(2.11)$ at $d \in \tilde{M}$.

We note as in Step 2 that

$$
\pi_{0}\left(\alpha^{\prime}\left(W_{\epsilon} \cap V_{0}\right)\right) \supset B\left(c_{0}, r\right) \cap V_{0}
$$

where $W_{\epsilon}$ has been defined in Step 2. Thus we can find a sequence $\left\{d_{i}\right\}$ such that (2.44) is satisfied and, moreover, if $P$ is the projection on $V_{0} \cap v^{\perp}$ and $v_{2} \in V_{0} \cap v^{\perp}$, we can ask

$$
\frac{P\left(-\alpha^{\prime}\left(d_{i}\right)+\alpha^{\prime}(c)\right)}{\left\|P\left(-\alpha^{\prime}\left(d_{i}\right)+\alpha^{\prime}(c)\right)\right\|} \rightarrow v_{2}
$$

We let $\tilde{M}$ the set of all the $d$ which are limits of such sequences $\left\{d_{i}\right\}$. We have seen after formula (2.44) that $\tilde{M} \subset M$. We set $\mathcal{B}_{i}=M_{-\alpha^{\prime}\left(d_{i}\right)}^{r e c}$ and we consider the exact forms $\eta_{\left(\mathcal{B}_{i}, d_{i}\right)}$ given by Step 3 ; using (2.44), it is easy to see that the elements of $\mathcal{B}_{i}$ converge to a set containing the $\left(-\alpha^{\prime}(c), v_{1}\right)$-lamination $\mathcal{B}$, and thus the limit $\eta_{(\mathcal{B}, d)}$ of $\eta_{\left(\mathcal{B}_{i}, d_{i}\right)}$ satisfies $(2.10)$ on $\mathcal{B}$. Formula (2.11) follows because the uniform limit of Lipschitz functions 
is Lipschitz. The fact that the closure of $\operatorname{co}(\tilde{M})$ is $M$ follows as in Step 2, using the fact that we can choose $v_{2}$ as we want in $V_{0} \cap v^{\perp}$.

The proof for a $\left(-\alpha^{\prime}(c), v_{1}, \ldots, v_{s}\right)$-lamination is similar.

We define $\mathcal{A}_{c}^{\perp}$ as the linear subspace of all the elements of $H^{n}\left(\mathbf{T}^{n+1}\right)$ which, for any $\left(-\alpha^{\prime}(c), v_{1}, \ldots, v_{s}\right)$ lamination $\mathcal{B}$ at $c$, have a representative vanishing on $\mathcal{B}$.

Lemma 2.9. Let $D_{\rho}$ be a flat of $\alpha$ of slope $\rho$, and let $D$ be a face of $D_{\rho}$, or a face of a face of $D_{\rho}$, etc... Let $\left(c_{0}, \alpha\left(c_{0}\right)\right) \in D^{\circ}$, and let $F_{D}$ denote the linear space generated by $D$. Then $F_{D} \subset \mathcal{A}_{c_{0}}^{\perp}$; in other words, if $\mathcal{B} \subset \mathcal{A}_{c_{0}}$ is a $\left(-\alpha^{\prime}\left(c_{0}\right), v_{1}, \ldots, v_{s}\right)$-lamination at $c_{0}$, then any element $\left(c+\alpha(c) \widehat{\mathrm{d} x_{n+1}}\right) \in F_{D}$ has a representative $\eta$ vanishing on it.

Proof. We note that $F_{D}$ is the space generated by $\left(c_{1}-c_{2}, \alpha\left(c_{1}\right)-\alpha\left(c_{2}\right)\right)$, with $\left(c_{i}, \alpha\left(c_{i}\right)\right) \in D^{\circ}$; thus it suffices to prove that $c_{1}-c_{2}+\left(\alpha\left(c_{1}\right)-\alpha\left(c_{2}\right)\right) \widehat{\mathrm{d} x_{n+1}}$ has a representative vanishing on $\mathcal{B}$ when $\left(c_{i}, \alpha\left(c_{i}\right)\right) \in D^{\circ}$.

By Lemma 2.5, we have that $\mathcal{B}$ is a $\left(-\alpha^{\prime}(c), v_{1}, \ldots, v_{s}\right)$-lamination both at $c_{1}$ and at $c_{2}$. We can apply Lemma 2.8 at $c_{1}$ and $c_{2}$, getting two exact forms $\eta_{\left(\mathcal{B}, c_{1}\right)}$ and $\eta_{\left(\mathcal{B}, c_{2}\right)}$ that satisfy $(2.10)$ for $f_{c_{1}}$ and $f_{c_{2}}$ respectively. Setting

$$
\begin{aligned}
& \tilde{\eta}_{1}=c_{1}+\eta_{\left(\mathcal{B}, c_{1}\right)}+\alpha\left(c_{1}\right) \widehat{\mathrm{d} x_{n+1}} \\
& \tilde{\eta}_{2}=c_{2}+\eta_{\left(\mathcal{B}, c_{2}\right)}+\alpha\left(c_{2}\right) \widehat{\mathrm{d} x_{n+1}}
\end{aligned}
$$

we immediately get that $\tilde{\eta}_{1}-\tilde{\eta}_{2}$ represents $\left(c_{1}+\alpha\left(c_{1}\right) \widehat{\mathrm{d} x_{n+1}}\right)-\left(c_{2}+\alpha\left(c_{2}\right) \widehat{\mathrm{d} x_{n+1}}\right)$; moreover, since $\left.\eta_{\left(\mathcal{B}, c_{i}\right.}\right)$ satisfies (2.10) for $f_{c_{i}}$, an easy calculation yields

$$
\left\{\begin{aligned}
\tilde{\eta}_{i}^{x}(x, u(x)) & =\partial_{p} \mathcal{L}(x, u(x), \nabla u(x)) \\
\tilde{\eta}_{i}^{u}(x) & =\mathcal{L}(x, u(x), \nabla u(x))-\left\langle\partial_{p} \mathcal{L}(x, u(x), \nabla u(x)), \nabla u(x)\right\rangle
\end{aligned}\right.
$$

for $u \in \mathcal{B}, x \in \mathbf{R}^{n}$ and $i=1,2$. But this implies that $\tilde{\eta}_{1}-\tilde{\eta}_{2}$ vanishes on $\mathcal{B}$, which is the thesis.

Lemma 2.10. Let $D$ be either the flat $D_{\rho}$, or one of its faces, subfaces, etc. Let $c \in D^{\circ}$, and let us suppose that $\mathcal{B}$ is $a\left(-\alpha^{\prime}(c), v_{1}, \ldots, v_{s}\right)$-lamination at $c$. Then $F_{D}$, the vector space generated by $D$, satisfies

$$
F_{D} \subset \operatorname{rat}\left(-\alpha^{\prime}(c), 1\right) \cap \operatorname{rat} v_{1} \cap \ldots \cap v_{s}
$$

If $\mathcal{B}$ is a foliation, then $F_{D}=\{0\}$.

Proof. We must prove that, if $\left(c_{1}, \alpha\left(c_{1}\right)\right),\left(c_{2}, \alpha\left(c_{2}\right)\right)$ are two elements of $D$, then

$$
\left(c_{1}-c_{2}, \alpha\left(c_{1}\right)-\alpha\left(c_{2}\right)\right) \in \operatorname{rat}\left(-\alpha^{\prime}(c), 1\right) \cap \operatorname{rat} v_{1} \cap \ldots \cap v_{s} .
$$

As in Lemma 2.9, we can as well suppose that $\left(c_{i}, \alpha\left(c_{i}\right)\right) \in D^{\circ}$. As usual, we shall suppose that

$$
\pi_{-1}\left[\operatorname{rat}\left(-\alpha^{\prime}(c), 1\right) \cap \operatorname{rat} v_{1} \cap \ldots \cap v_{s}\right]=\mathbf{R}^{j} \times\{0\} .
$$

By Lemma 2.9, $c_{1}-c_{2}+\left(\alpha\left(c_{1}\right)-\alpha\left(c_{2}\right)\right) \widehat{\mathrm{d} x_{n+1}}$ has a representative vanishing on $\mathcal{B}$. If $\mathcal{B}$ is a foliation, we have that this representative vanishes everywhere, i.e. $F_{D}=\{0\}$, and the thesis follows. If $\mathcal{B}$ is not a foliation, we consider one of its gaps, say $A$; let $H_{1}(i)$ be the map form $H_{1}(A)$ to $H_{1}\left(\mathbf{T}^{n+1}\right)$ induced by the injection $i: A \rightarrow \mathbf{T}^{n+1}$. By Alexander duality, the space of all forms vanishing on $\mathcal{B}$ is isomorphic to the image of $H_{1}(i)$; in particular, it has dimension $j$. Thus, the thesis follows if we prove that this space contains the space generated by $\widehat{\mathrm{d} x_{1}}+\rho_{1} \mathrm{~d} x_{n+1}, \ldots, \widehat{\mathrm{d} x_{j}}+\rho_{j} \widehat{\mathrm{d} x_{n+1}}$ : since the dimension is the same, they must coincide.

To prove this, we note that, since $\mathcal{B}$ is closed by Lemma 2.12 below, the gap $A$ is bounded by two elements of $\mathcal{B}$, say $\underline{u}<\bar{u}$. Let $-\alpha^{\prime}(c)=\left(\frac{p_{1}}{q_{1}}, \ldots, \frac{p_{j}}{q_{j}}, \rho_{j+1}, \ldots, \rho_{n}\right)$; from the last formula above we deduce easily that $\underline{u}$ 
and $\bar{u}$ are periodic in the directions $e_{1}, \ldots, e_{j}$; in other words, $\underline{u}\left(x+q_{i} e_{i}\right)=\underline{u}(x)+p_{i}$ and $\bar{u}\left(x+q_{i} e_{i}\right)=\bar{u}(x)+p_{i}$ for $i \in(1, \ldots, j)$. From this it follows that, if $\epsilon$ is small enough, the loops

$$
\gamma_{i}: t \rightarrow e_{i} t+\left[\underline{u}\left(t e_{i}\right)+\epsilon\right] e_{n+1} \quad i \in(1, \ldots, j)
$$

have image in the gap and their projection on $\mathbf{T}^{n+1}$ is closed.

We now assert that the vector space of all closed $n$-forms vanishing on the boundary of the gap contains

$$
\left\{\left(c, c_{n+1}\right): c \in \mathbf{R}^{j} \times\{0\}, c_{n+1}=\rho \cdot c\right\}
$$

for the base $\widehat{\mathrm{d} x_{1}}, \ldots, \widehat{\mathrm{d} x_{n+1}}$. To prove this, we consider $M=S^{1} \times[-1,1]^{n}$ with coordinates $x_{1} \in S^{1}$, $x_{2}, \ldots, x_{n+1} \in[-1,1]^{n}$; on $M$ we define the vector field $v=e_{1} \psi\left(x_{2}, \ldots, x_{n+1}\right)$ with $\psi$ a cutoff compactly supported in $[-1,1]^{n}$. Since $\operatorname{div} v=0$, the $n$-form $\omega=\psi \widehat{\mathrm{d} x_{1}}$ corresponding to $v$ is closed; moreover, if $\psi$ has integral 1, we have

$$
\int_{\left\{x_{i}=0\right\}} \omega=\left\{\begin{array}{lll}
1 & \text { if } \quad i=1 \\
0 & \text { if } \quad i \neq 1
\end{array}\right.
$$

We now consider a neighbourhood of the loop $\gamma_{i}$ all contained in $\mathcal{B}$; we send $M$ into this neighbourhood by a diffeo $\phi_{i}$. If we extend $\phi_{i}^{*}(\omega)$ to zero outside the image of $M$, we get a closed form $\omega_{i}$, supported in a gap of $\mathcal{B}$ such that $\left[\omega_{i}\right]=\widehat{\mathrm{d} x_{i}}+\rho_{i} \widehat{\mathrm{d} x_{n+1}}$; this proves the assertion.

The next lemma gives information on the structure of the Aubry set; we have used part of it in the proof of Lemma 2.8.

Lemma 2.11. Let $c \in H$, and let $\mathcal{B} \subset \mathcal{A}_{c}$ be $a\left(-\alpha^{\prime}(c), v_{1}, \ldots, v_{s}\right)$-lamination. Then

1) $\mathcal{B}$ is closed in the $C_{\text {loc }}^{1}$ topology.

2) $\mathcal{B}$ contains all the $u \in M_{-\alpha^{\prime}(c)}$ with $l(u) \leq s$ and $v_{i}(u)=v_{i}$ for $i \leq l(u)$.

3) If $n=1, \mathcal{B}$ contains the Aubry set of [16].

Proof. We begin to prove that $\mathcal{B}$ is closed. Let $\left\{u_{i}\right\} \subset \mathcal{B}$ satisfy

$$
u_{i} \rightarrow u \quad \text { in } \quad C_{l o c}^{1}\left(\mathbf{R}^{n}\right) \quad \text { as } \quad i \rightarrow+\infty
$$

We have to prove that $u \in \mathcal{B}$. Since $u_{i}$ has invariants $\left(-\alpha^{\prime}(c), v_{1}, \ldots, v_{s_{i}^{\prime}}\right)$ with $s_{i}^{\prime} \leq s$, the remarks at the beginning of this section imply that $u$ has invariants $\left(-\alpha^{\prime}(c), v_{1}, \ldots, v_{l}\right)$ with $l \leq \liminf s_{i}^{\prime} \leq s$. Since $u_{i} \in \mathcal{B}$, we can find $u_{k, i} \in M_{\alpha^{\prime}\left(d_{k, i}\right)}^{r e c}$ such that

$$
\begin{gathered}
u_{k, i} \rightarrow u_{i} \quad \text { in } C_{l o c}^{1}\left(\mathbf{R}^{n}\right) \quad \text { as } k \rightarrow+\infty \\
\left|A\left(T_{k, i}\right)-T_{k, i}\left(\eta_{c}\right)-\alpha(c)\right| \leq \delta\left\|\pi_{s_{i}^{\prime}-1}\left(\alpha^{\prime}\left(d_{k, i}\right)-\alpha^{\prime}(c)\right)\right\| \quad \text { for } k \text { large }
\end{gathered}
$$

where $T_{k, i}$ denotes the current induced by $u_{k, i}$. By $(2.45)$ and the last two formulas, we get that there is $k(i)$ such that

$$
\begin{aligned}
u_{k(i), i} & \rightarrow u \quad \text { in } \quad C_{l o c}^{1}\left(\mathbf{R}^{n}\right) \quad \text { as } i \rightarrow+\infty \\
\left|A\left(T_{k(i), i}\right)-T_{k(i), i}\left(\eta_{c}\right)-\alpha(c)\right| & \leq \delta\left\|\pi_{s_{i}^{\prime}-1}\left(\alpha^{\prime}\left(d_{k(i), i}\right)-\alpha^{\prime}(c)\right)\right\| \leq\left\|\pi_{l-1}\left(\alpha^{\prime}\left(d_{k(i), i}\right)-\alpha^{\prime}(c)\right)\right\|
\end{aligned}
$$

where the last inequality follows from the fact that $l \leq \liminf s_{i}^{\prime}$. The last two formulas imply that $u \in \mathcal{B}$, i.e. that $\mathcal{B}$ is closed.

Let now $\mathcal{B}$ be as above, and let $w \in M_{-\alpha^{\prime}(c)}$ have invariants $-\alpha^{\prime}(c), v_{1}, \ldots, v_{s^{\prime}}$ with $s^{\prime} \leq s$; we must prove that $w \in \mathcal{B}$. For starters, we prove this when $s \geq 1$. Let $\left(d_{i}, u_{i}\right)$ satisfy $(2.3)$ and $(2.4)$ and let the limit $u \in \mathcal{B}$ have invariants $\left(-\alpha^{\prime}(c), v_{1}, \ldots, v_{s}\right)$; such a $u$ exists by the definition of $\left(-\alpha^{\prime}(c), v_{1}, \ldots, v_{s}\right)$-lamination. We begin to show that $w$ is approximated by suitable translates of $u_{i}$. We note that $\alpha^{\prime}\left(d_{i}\right) \neq \alpha^{\prime}(c)$ : otherwise, $l(u)=s=0$, while we are supposing that $s \geq 1$. But, if $\alpha^{\prime}\left(d_{i}\right) \neq \alpha^{\prime}(c)$, then $u_{i} \in M_{-\alpha^{\prime}\left(d_{i}\right)}^{r e c}$ intersects $w$; if instead of $u_{i}$ 
we consider $u_{i}\left(x+k_{i}\right)+j_{i}$, we can suppose that the intersection point $x_{i}$ is bounded as $i \rightarrow+\infty$. Now by (3) of the introduction and the Ascoli-Arzelà theorem we get that, up to a subsequence, $u_{i}\left(\cdot+k_{i}\right)+j_{i} \rightarrow \bar{u}$

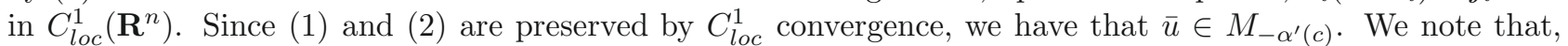
since $u_{i}\left(x_{i}+k_{i}\right)+j_{i}=w\left(x_{i}\right)$ and $x_{i}$ is bounded, then $\bar{u}\left(x_{0}\right)=w\left(x_{0}\right)$ for some $x_{0} \in \mathbf{R}^{n}$. If $\bar{u}=w$, then we have that $w \in \mathcal{B}$; indeed, this means that $u_{i}\left(\cdot+k_{i}\right)+j_{i} \rightarrow w$ in $C_{l o c}^{1}$, which is (2.3). Since $u$ has invariants $-\alpha^{\prime}(c), v_{1}, \ldots, v_{s}$, since $\left(d_{i}, u_{i}\right)$ satisfies $(2.4)$ and $s^{\prime} \leq s$, we get that

$$
\left|\alpha\left(d_{i}\right)+\left\langle\alpha^{\prime}\left(d_{i}\right), c-d_{i}\right\rangle-\alpha(c)\right| \leq \delta\left\|\pi_{s^{\prime}-1}\left(\alpha^{\prime}\left(d_{i}\right)-\alpha^{\prime}(c)\right)\right\|
$$

which is (2.4) for $w$.

To prove that $\bar{u}=w$, we use the result of [4] we mentioned at the beginning of this section: if $\bar{u}, w \in M_{-\alpha^{\prime}(c)}$, if $\bar{u}\left(x_{0}\right)=w\left(x_{0}\right)$ and if the invariants of $\bar{u}$ are contained in, or contain the invariants of $w$, then $\bar{u}=w$. We have already seen that $\bar{u}\left(x_{0}\right)=w\left(x_{0}\right)$. To determine the relation between the invariants, we note that $u$ is approximated by $\left\{u_{i}\right\}$ and $\bar{u}$ by $\left\{u_{i}\left(x+k_{i}\right)+j_{i}\right\}$; by the remarks at the beginning of this section, we have that the invariants of $\bar{u}$ are contained in, or contain, those of $u$, which is exactly what we wanted to prove.

To prove point 2) when $s=0$, we begin to show that $M_{-\alpha^{\prime}(c)}^{r e c} \subset \mathcal{B}$; it suffices to show that $u \in M_{-\alpha^{\prime}(c)}^{r e c}$ satisfies (2.3) and (2.4). But this follows immediately if we take $d_{i} \equiv c$ and $u_{i} \equiv u$. This implies that, if $-\alpha^{\prime}(c) \in \mathbf{Q}^{n}$ then $\mathcal{B}$ contains all the $u \in M_{-\alpha^{\prime}(c)}$ with $l(u)=0$; this is because, when $-\alpha^{\prime}(c) \in \mathbf{Q}^{n}$, it is easy to see that $l(u)=0$ iff $u$ is recurrent. Let now $-\alpha^{\prime}(c) \in \mathbf{R}^{n} \backslash \mathbf{Q}^{n}$; we know from Lemma 2.9 that the flat $D$ of $\alpha$ containing $c$ is contained in $\operatorname{rat}\left(-\alpha^{\prime}(c), 1\right)$. Since $-\alpha^{\prime}(c)$ has at least one irrational component, we have that $\pi_{-1}\left(\operatorname{rat}\left(-\alpha^{\prime}(c), 1\right)\right)$ has codimension at least 1 . But then by Lemma $2.9 \pi_{-1}(D)$ has codimension at least 1 . Thus $c \in \pi_{-1}(D)$ is accumulated by points outside $D$, i.e. we can find $d_{i} \rightarrow c$ with $-\alpha^{\prime}\left(d_{i}\right) \neq-\alpha^{\prime}(c)$. Now the elements $u_{i} \in M_{-\alpha^{\prime}\left(d_{i}\right)}^{r e c}$ converge to the elements of a $\left(-\alpha^{\prime}(c)\right)$-lamination by Lemma 2.3 ; moreover, since $-\alpha^{\prime}\left(d_{i}\right) \neq-\alpha^{\prime}(c)$, they intersect any $u \in M_{-\alpha^{\prime}(c)}$, among which the ones with $l(u)=0$; now the same argument as above yields that $\left\{u_{i}\right\}$ converges, up to a subsequence, to $u$, i.e. $u \in \mathcal{B}$.

To prove point 3), we distinguish various cases. In the first one, $\rho \in \mathbf{R} \backslash \mathbf{Q}$; but then $M_{\rho}$ is ordered and all its elements $u$ have $l(u)=0$; by point 2), this implies that $M_{\rho}$ is the Aubry set at $c$; since $M_{\rho}$ is the Aubry set also in the sense of [16], we have done in this case. The other cases are when $\rho \in \mathbf{Q}$ and

$$
D_{\rho}=\left\{(c, \alpha(c)): c \in\left[c_{1}, c_{2}\right]\right\} .
$$

If $c=c_{1}$, then it is easy to see that the limits of sequences $u_{i} \in M_{-\alpha^{\prime}\left(d_{i}\right)}$ with $\left\{d_{i}\right\}$ satisfying $(2.4)$ at $c=c_{1}$ have invariants $(\rho,(-1, \rho))$; by point 2$)$, this implies that our Aubry set is $M_{(\rho,(-1, \rho))}$, which is also the Aubry set in the sense of [16]. The situation at $c_{2}$ is analogous. If $c \in\left(c_{1}, c_{2}\right)$, we see easily that our Aubry set is $M_{-\alpha^{\prime}(c)}^{r e c}$, which again coincides with the Aubry set of [16].

\subsection{The flat extends into the rational space}

We begin with a lemma about the shape of $D_{\rho}$ for rational frequencies.

Lemma 2.12. Let us suppose that $\rho^{i} \in \mathbf{Q}^{n}$ and that

$$
\rho^{i}=\left(\frac{p_{1}}{q_{1}}, \ldots, \frac{p_{L}}{q_{L}}, \rho_{i}^{L+1}, \ldots, \rho_{i}^{n}\right)
$$

Let $\rho, v_{1}, \ldots, v_{t}$ be admissible and let us denote by $\mathcal{C}$ the lamination of all the $u \in M_{\rho}$ with $l(u) \leq t$ and $v_{i}(u)=v_{i}$ for $i \leq l(u)$. Let us suppose that the gaps of $\mathcal{C}$ contain elements of $M_{\rho}$ with different invariants or, equivalently, that $M_{\left(\rho, v_{1}, \ldots, v_{t}\right)}$ is not ordered. Let us suppose that the elements of $M_{\rho^{i}}^{r e c}$ converge to elements of $\mathcal{C}$, and let $D_{\rho^{i}}$ be the flat of $\alpha$ of slope $\rho^{i}$. Then there is $\gamma>0$ such that the following holds: if $j \in(1, \ldots, L)$ and if $i$ is large enough, then we can find $(d, \alpha(d)),\left(d^{\prime}, \alpha\left(d^{\prime}\right)\right) \in D_{\rho^{i}}$ such that

$$
\left|\left\langle e_{j},(d, \alpha(d))-\left(d^{\prime}, \alpha\left(d^{\prime}\right)\right)\right\rangle\right| \geq \gamma .
$$


Proof. Let us pick $d_{i}$ with $-\alpha^{\prime}\left(d_{i}\right)=\rho_{i}$; as usual, we consider the Lagrangian $f_{d_{i}}$ and the related $\alpha$-function $\alpha_{i}$; the latter satisfies $\alpha_{i}(0)=0,-\alpha_{i}^{\prime}(0)=\rho^{i}$. We call $A_{i}$ the mean action of $f_{d_{i}}$ and we suppose for the moment that $q_{1}$ is relatively prime to the other denominators.

\section{Step 1: Heteroclinics in $M_{\rho^{i}}$.}

Let $A_{\Gamma_{i}} \subset \mathbf{R}^{n-1}$ be a fundamental domain for $\left(\frac{p_{2}}{q_{2}}, \ldots, \frac{p_{L}}{q_{L}}, \rho_{i}^{L+1}, \ldots, \rho_{i}^{n}\right)$. Since $q_{1}$ is relatively prime to the other denominators, $\left[0, q_{1}\right] \times A_{\Gamma_{i}}$ is a fundamental domain for $\rho^{i}$ by Lemma A2 of the Appendix. Let $\left(k_{i}, j_{i}\right) \in \mathbf{Z}^{n} \times \mathbf{Z}$ be such that

$$
\gamma_{i}=\rho^{i} \cdot k_{i}+j_{i}>0
$$

be minimal. We get as in formula (2.30) that

$$
\frac{1}{\gamma_{i}}=q_{1}\left|A_{\Gamma_{i}}\right|=l_{i} \in \mathbf{N}
$$

We set

and we select $u_{ \pm}^{i, s} \in M_{\rho_{ \pm}^{i, s}}^{r e c}$. We have that $u_{ \pm}^{i, s}$ satisfies

$$
\rho_{ \pm}^{i, s}=\rho^{i} \pm \frac{1}{q_{1} s} e_{1}
$$

$$
u_{ \pm}^{i, s}\left(x+q_{1} s e_{1}\right)=u_{ \pm}^{i, s}(x)+p_{1} s \pm 1 .
$$

Our first step consist in fixing $i$ and letting $s \rightarrow+\infty$; we shall study the limits of $u_{ \pm}^{i, s}$ as $s \rightarrow+\infty$. Let $u_{i} \in M_{\rho^{i}}^{r e c}$; from point 4) of Lemma A1 we see that, for $l_{i}$ defined as in (2.46),

$$
u_{i}\left(x+l_{i} k_{i}\right)+l_{i} j_{i}=u_{i}(x)+1 .
$$

For $0 \leq m<l_{i}$, let us define

$$
G_{m}^{+}=\left\{x \in \mathbf{R}^{n}: u_{i}\left(x+m k_{i}\right)+m j_{i} \leq u_{+}^{i, s}(x)<u_{i}\left(x+(m+1) k_{i}\right)+(m+1) j_{i},\right.
$$

By the last three formulae, we get that

$$
\left.\left(x_{2}, \ldots, x_{n}\right) \in A_{\Gamma_{i}}\right\} .
$$

$$
\bigcup_{m=0}^{l_{i}-1} G_{m}^{+}=\left\{x: u_{i}(x) \leq u_{+}^{i, s}(x)<u_{i}(x)+1\right\}
$$

is a fundamental domain for $u_{+}^{i, s}$, equivalent to $\left[0, s q_{1}\right] \times A_{\Gamma_{i}}$. We define analogously $G_{m}^{-}$for $u_{-}^{i, s}$. Now

$$
\begin{aligned}
& \int_{\left[0, s q_{1}\right] \times A_{\Gamma_{i}}}\left[\mathcal{L}\left(x, u_{+}^{i, s}, \nabla u_{+}^{i, s}\right)+\mathcal{L}\left(x, u_{-}^{i, s}, \nabla u_{-}^{i, s}\right)\right] \mathrm{d} x= \\
& \sum_{m=0}^{l_{i}-1}\left[\int_{G_{m}^{+}} \mathcal{L}\left(x, u_{+}^{i, s}, \nabla u_{+}^{i, s}\right) \mathrm{d} x+\int_{G_{m}^{-}} \mathcal{L}\left(x, u_{-}^{i, s}, \nabla u_{-}^{i, s}\right) \mathrm{d} x\right] .
\end{aligned}
$$

We now show that we can translate $u_{+}^{i, s}$ and $u_{-}^{i, s}$ in such a way that they intersect in $\mathcal{G}_{m}^{+} \cap \mathcal{G}_{m}^{-}$; we also want the last formula to hold for the translates.

For $r \in \mathbf{N}$, let

$$
\begin{gathered}
\tilde{G}_{m}^{+}=\left\{x \in \mathbf{R}^{n}: u_{i}\left(x+m k_{i}\right)+m j_{i} \leq u_{+}^{i, s}\left(x+r q_{1} e_{1}\right)-r p_{1}<u_{i}\left(x+(m+1) k_{i}\right)+(m+1) j_{i},\right. \\
\left.\left(x_{2}, \ldots, x_{n}\right) \in A_{\Gamma_{i}}\right\} .
\end{gathered}
$$


It is easy to see that $\tilde{G}_{m}^{+}=G_{m}^{+}-r q_{1} e_{1}$; moreover, $u_{+}^{i, s}\left(x+r q_{1} e_{1}\right)-\left.r p_{1}\right|_{\tilde{G}_{m}^{+}}$is a translate of $\left.u_{+}^{i, s}\right|_{\tilde{G}_{m}^{+}}$. This implies that we can translate $u_{ \pm}^{i, s}$ in such a way that they intersect between $u_{i}\left(x+m k_{i}\right)+m j_{i}$ and $u_{i}(x+(m+$ $\left.1) k_{i}\right)+(m+1) j_{i}$; the strip between these two periodic solutions translates to itself and the action of the portion of $u_{ \pm}^{i, s}$ contained in the strip remains unchanged. Let us call $x^{i, s}$ a point of intersection; we can also require that $x^{i, s}$ is bounded for $s \in \mathbf{Z}$, and that the distance of $\left(x^{i, s}, u_{ \pm}^{i, s}\left(x^{i, s}\right)\right)$ from the graphs of $u_{i}\left(x+m k_{i}\right)+m j_{i}$ and $u_{i}\left(x+(m+1) k_{i}\right)+(m+1) j_{i}$ is bounded away from 0. Using (3) and Ascoli-Arzelà we get that there are $u_{+}^{i}, u_{-}^{i} \in M_{\alpha^{\prime}(c)}$ such that

$$
u_{+}^{i, s} \rightarrow u_{+}^{i}, \quad u_{-}^{i, s} \rightarrow u_{-}^{i} \quad \text { in } \quad C_{l o c}^{1}\left(\mathbf{R}^{n}\right) \quad \text { as } s \rightarrow+\infty
$$

It is easy to see that $u_{+}^{i}$ has invariants $\rho_{i},\left(e_{1},-\rho_{1}\right)$, and $u_{-}^{i}$ has invariants $\rho_{i},\left(-e_{1}, \rho_{1}\right)$. In other words, $u_{ \pm}^{i}$ are heteroclinics living in the gap

$$
\left\{\left(x, x_{n+1}\right): u_{i}\left(x+m k_{i}\right)+m j_{i}<x_{n+1}<u_{i}\left(x+(m+1) k_{i}\right)+(m+1) j_{i}\right\} .
$$

We also note that $u_{+}^{i} \neq u_{-}^{i}$, since they have different invariants.

\section{Step 2: Action estimates.}

We now use an argument of [17] and [19]: we set

$$
\left\{\begin{array}{l}
u_{\max }(x)=\max \left(u_{+}^{i}(x), u_{-}^{i}(x)\right) \\
u_{\min }(x)=\min \left(u_{+}^{i}(x), u_{-}^{i}(x)\right) .
\end{array}\right.
$$

Let $x_{1} \in G_{m}^{+} \cap G_{m}^{-}$and $\delta>0$ be such that $u_{\max }\left(x_{1}\right)=u_{\min }\left(x_{1}\right)$, but $u_{\max }$ and $u_{\min }$ do not coincide in $B\left(x_{1}, \delta\right) \subset$ $G_{m}^{+} \cap G_{m}^{-}$; since $u_{\max } \geq u_{\min }$, by the maximum principle they cannot be both solutions of $(4)$ in $B\left(x_{1}, \delta\right)$. In particular, they cannot both be minimal in $B\left(x_{1}, \delta\right)$. Let us suppose to fix ideas that $u_{\max }$ is not minimal; thus we can find $\phi_{i, m} \in C_{0}^{\infty}\left(B\left(x_{1}, \delta\right)\right)$ and $a_{i, m}>0$ such that

$$
\int_{B\left(x_{1}, \delta\right)} \mathcal{L}\left(x, u_{\max }, \nabla u_{\max }\right) \mathrm{d} x-2 a_{i, m} \geq \int_{B\left(x_{1}, \delta\right)} \mathcal{L}\left(x, u_{\max }+\phi_{i, m}, \nabla\left(u_{\max }+\phi_{i, m}\right)\right) \mathrm{d} x .
$$

Since

$$
\int_{B\left(x_{1}, \delta\right)} d_{i} \cdot \nabla \phi \mathrm{d} x=0
$$

by the divergence theorem, we can as well write

$$
\int_{B\left(x_{1}, \delta\right)} f_{d_{i}}\left(x, u_{\max }, \nabla u_{\max }\right) \mathrm{d} x-2 a_{i, m} \geq \int_{B\left(x_{1}, \delta\right)} f_{d_{i}}\left(x, u_{\max }+\phi_{i, m}, \nabla\left(u_{\max }+\phi_{i, m}\right)\right) \mathrm{d} x .
$$

Actually, by Lemma 2.8 we can add an exact form to $f_{d_{i}}$ in such a way that

$$
f_{d_{i}}\left(x, u_{i}(x), \nabla u_{i}(x)\right) \equiv 0
$$

Since

$$
\int_{B\left(x_{1}, \delta\right)}\left[f_{d_{i}}\left(x, u_{+}^{i}, \nabla u_{+}^{i}\right)+f_{d_{i}}\left(x, u_{-}^{i}, \nabla u_{-}^{i}\right)\right] \mathrm{d} x=\int_{B\left(x_{1}, \delta\right)}\left[f_{d_{i}}\left(x, u_{\max }^{i}, \nabla u_{\max }^{i}\right)+f_{d_{i}}\left(x, u_{\min }^{i}, \nabla u_{\min }^{i}\right)\right] \mathrm{d} x
$$




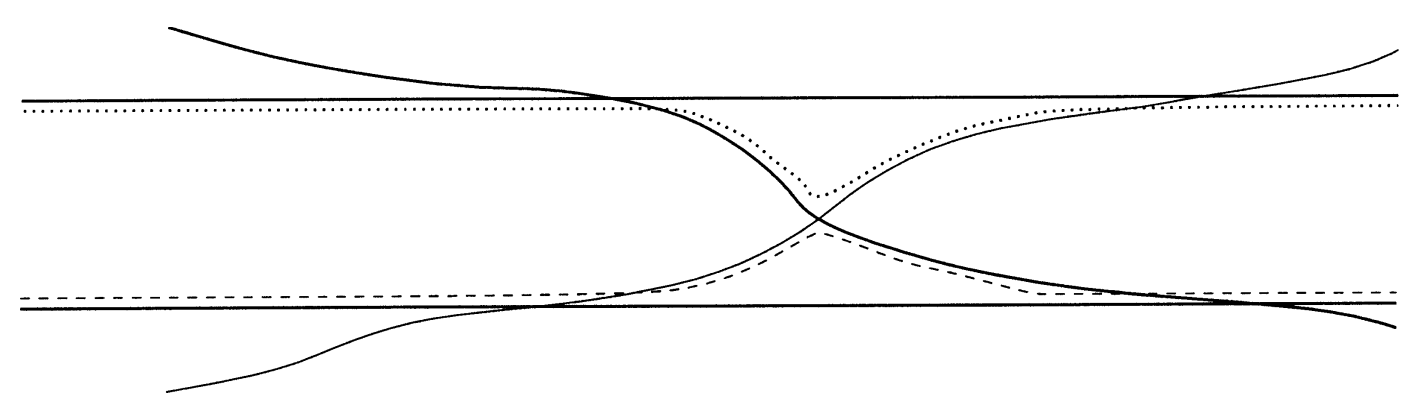

Figure 1. The dotted and dashed lines cannot both be minimizers.

we have that

$$
\begin{aligned}
\int_{B\left(x_{1}, \delta\right)}\left[f_{d_{i}}\left(x, u_{+}^{i}, \nabla u_{+}^{i}\right)+f_{d_{i}}\left(x, u_{-}^{i}, \nabla u_{-}^{i}\right)\right] \mathrm{d} x-2 a_{i, m} \geq \\
\int_{B\left(x_{1}, \delta\right)}\left[f_{d_{i}}\left(x, u_{\max }^{i}+\phi_{i, m}, \nabla\left(u_{\max }^{i}+\phi_{i, m}\right)\right)+f_{d_{i}}\left(x, u_{\min }^{i}, \nabla u_{\min }^{i}\right)\right] \mathrm{d} x
\end{aligned}
$$

Let us define

$$
\left\{\begin{array}{l}
u_{\max }^{i, m}(x)=\min \left[\max \left(u_{+}^{i, s}(x), u_{-}^{i, s}(x)\right), u_{i}\left(x+(m+1) k_{i}\right)+(m+1) j_{i}\right] \\
u_{\min }^{i, m}(x)=\max \left[\min \left(u_{+}^{i, s}(x), u_{-}^{i, s}(x)\right), u_{i}\left(x+m k_{i}\right)+m j_{i}\right]
\end{array}\right.
$$

We refer the reader to Figure 1: $u_{i}\left(x+m k_{i}\right)+m j_{i}$ and $u_{i}\left(x+(m+1) k_{i}\right)+(m+1) j_{i}$ are the two continuous horizontal lines, $u_{+}^{i, s}$ and $u_{-}^{i, s}$ are the two continuous slanted lines, $u_{\text {max }}^{i, m}$ is the dotted line and $u_{\text {min }}^{i, m}$ is the dashed line.

We have that, for $i$ large,

$$
\begin{aligned}
\int_{G_{m}^{+}} f_{d_{i}}\left(x, u_{+}^{i, s}, \nabla u_{+}^{i, s}\right) \mathrm{d} x+\int_{G_{m}^{-}} f_{d_{i}}\left(x, u_{-}^{i, s}, \nabla u_{-}^{i, s}\right) \mathrm{d} x=\int_{G_{m}^{+} \backslash B\left(x_{1}, \delta\right)} f_{d_{i}}\left(x, u_{+}^{i, s}, \nabla u_{+}^{i, s}\right) \mathrm{d} x \\
+\int_{G_{m}^{-} \backslash B\left(x_{1}, \delta\right)} f_{d_{i}}\left(x, u_{-}^{i, s}, \nabla u_{-}^{i, s}\right) \mathrm{d} x+\int_{B\left(x_{1}, \delta\right)}\left[f_{d_{i}}\left(x, u_{+}^{i, s}, \nabla u_{+}^{i, s}\right)+f_{d_{i}}\left(x, u_{+}^{i, s}, \nabla\left(u_{+}^{i, s}\right)\right)\right] \mathrm{d} x \\
\geq \int_{G_{m}^{+} \backslash B\left(x_{1}, \delta\right)} f_{d_{i}}\left(x, u_{+}^{i, s}, \nabla u_{+}^{i, s}\right) \mathrm{d} x+\int_{G_{m}^{-} \backslash B\left(x_{1}, \delta\right)} f_{d_{i}}\left(x, u_{-}^{i, s}, \nabla u_{-}^{i, s}\right) \mathrm{d} x \\
\quad+\int_{B\left(x_{1}, \delta\right)}\left[f_{d_{i}}\left(x, u_{\max }^{i, m}+\phi_{m}, \nabla\left(u_{\max }^{i, m}+\phi_{m}\right)\right)+f_{d_{i}}\left(x, u_{\min }^{i, m}, \nabla u_{\min }^{i, m}\right)\right] \mathrm{d} x+a_{i, m} \\
=\int_{G_{m}^{+}} f_{d_{i}}\left(x, u_{\max }^{i, m}+\phi_{i, m}, \nabla\left(u_{\max }^{i, m}+\phi_{i, m}\right)\right) \mathrm{d} x+\int_{G_{m}^{-}} f_{d_{i}}\left(x, u_{\min }^{i, m}, \nabla u_{\min }^{i, m}\right) \mathrm{d} x+a_{i, m} \\
=\int_{\left[0, s q_{1}\right] \times A_{\Gamma_{i}}}\left[f_{d_{i}}\left(x, u_{\max }^{i, m}+\phi_{i, m}, \nabla\left(u_{\max }^{i, m}+\phi_{i, m}\right)\right)+f_{d_{i}}\left(x, u_{\min }^{i, m}, \nabla u_{\min }^{i, m}\right)\right] \mathrm{d} x+a_{i, m}
\end{aligned}
$$

The first inequality of the formula above comes from (2.48) and (2.49), and the last equality comes from the fact that

$$
f_{d_{i}}\left(x, u_{i}, \nabla u_{i}\right) \equiv 0
$$


We have that

$$
\begin{gathered}
\int_{\left[0, s q_{1}\right] \times A_{\Gamma_{i}}}\left[f_{d_{i}}\left(x, u_{+}^{i, s}, \nabla u_{+}^{i, s}\right)+f_{d_{i}}\left(x, u_{-}^{i, s}, \nabla u_{-}^{i, s}\right)\right] \mathrm{d} x= \\
\sum_{m=0}^{l_{i-1}}\left[\int_{G_{m}^{+}} f_{d_{i}}\left(x, u_{+}^{i, s}, \nabla u_{+}^{i, s}\right) \mathrm{d} x+\int_{G_{m}^{-}} f_{d_{i}}\left(x, u_{-}^{i, s}, \nabla u_{-}^{i, s}\right) \mathrm{d} x\right] \\
\geq \sum_{m=0}^{l_{i}-1} \int_{\left[0, s q_{1}\right] \times A_{\Gamma_{i}}}\left\{\left[f_{d_{i}}\left(x, u_{\max }^{i, m}+\phi_{m}, \nabla\left(u_{\max }^{i, m}+\phi_{m}\right)\right)+f_{d_{i}}\left(x, u_{\min }^{i, m}, \nabla u_{\min }^{i, m}\right)\right] \mathrm{d} x+a_{i, m}\right\} .
\end{gathered}
$$

In the equality above, we have used the fact that $\cup_{m=0}^{l_{i}-1} \mathcal{G}_{m}^{ \pm}$are fundamental domains for $u_{ \pm}^{i, s}$ respectively; in the inequality, we have used (2.50).

\section{Step 3: Uniformity.}

Now let $\left(k_{i}^{\prime}, j_{i}^{\prime}\right) \in \mathbf{Z}^{n-1} \times \mathbf{Z}$ be such that

$$
\frac{1}{l_{i}^{\prime}}=\left(\frac{p_{2}}{q_{2}}, \ldots, \frac{p_{L}}{q_{L}}, \rho_{i}^{L+1}, \ldots, \rho_{i}^{n}\right) \cdot k_{i}^{\prime}+j_{i}^{\prime}>0
$$

be minimal. We know from Lemma A1 that $l_{i}^{\prime} \in \mathbf{N}$, and from (2.30) that $l_{i}^{\prime}=\left|A_{\Gamma_{i}}\right|$. We note that, by (2.46), $l_{i}=l_{i}^{\prime} q_{1}$. We now translate the four functions $u\left(x+m k_{i}\right)+m j_{i}, u\left(x+(m+1) k_{i}\right)+(m+1) j_{i}$ and $u_{ \pm}^{i, s}$ by $\left(k_{i}^{\prime}, j_{i}^{\prime}\right)$; we obtain $u\left(x+\left(m+q_{1}\right) k_{i}\right)+\left(m+q_{1}\right) j_{i}, u\left(x+\left(m+q_{1}+1\right) k_{i}\right)+\left(m+q_{1}+1\right) j_{i}$ and $u_{ \pm}^{i, s}\left(x+k_{i}^{\prime}\right)+j_{i}^{\prime}$. In other words, $\mathcal{G}_{m}$ and the two heteroclinics intersecting in $\mathcal{G}_{m}$ are brought to $\mathcal{G}_{m+q_{1}}$. As a consequence,

$$
a_{i, m}=a_{i, m+q_{1}}=\ldots=a_{i, m+\left(l_{i}^{\prime}-1\right) q_{1}} .
$$

If we denote by $\{m\}$ the equivalence class of $m$ modulo $q_{1}$, we get that $a_{i, m}=a_{i,\{m\}}$. Now we get from (2.51) that

$$
\begin{gathered}
\int_{\left[0, s q_{1}\right] \times A_{\Gamma_{i}}}\left[f_{d_{i}}\left(x, u_{+}^{i, s}, \nabla u_{+}^{i, s}\right)+f_{d_{i}}\left(x, u_{-}^{i, s}, \nabla u_{-}^{i, s}\right)\right] \mathrm{d} x \geq \\
\sum_{m=0}^{l_{i}-1}\left[\int_{\left[0, s q_{1}\right] \times A_{\Gamma_{i}}}\left[f_{d_{i}}\left(x, u_{\max }^{i, m}+\phi_{m}, \nabla\left(u_{\max }^{i, m}+\phi_{m}\right)\right)+f_{d_{i}}\left(x, u_{\min }^{m}, \nabla u_{\min }^{m}\right)\right] \mathrm{d} x+a_{i,\{m\}}\right] .
\end{gathered}
$$

Now it is easy to see that, for at least one equivalence class $\{\bar{m}\}$, the two solutions bounding $\mathcal{G}_{\bar{m}}$ converge in $C_{\text {loc }}^{1}$ to different elements of $\mathcal{C}$; otherwise, we would have that $u_{i}\left(x+k_{i}\right)+j_{i}-u_{i}(x) \rightarrow 0$, while we know that it is constantly 1 . But this implies that we can choose $\bar{m}$ is such a way that, on $\mathcal{G}_{\bar{m}}$, the heteroclinics $u_{ \pm}^{i}$ converge to intersecting heteroclinics, say $u_{ \pm}$, in a gap of $\mathcal{C}$ as $i \rightarrow+\infty$. Since $u_{ \pm}^{i, s}$ are close to $u_{ \pm}$on $B\left(x_{1}, \delta\right)$ for $i, s$ large, we get that $a_{i,\{\bar{m}\}} \geq a>0$. Since in an equivalence class there are $l_{i}^{\prime}=\left|A_{\Gamma_{i}}\right|$ elements, we get

$$
\begin{aligned}
\int_{\left[0, s q_{1}\right] \times A_{\Gamma_{i}}}\left[f_{d_{i}}\left(x, u_{+}^{i, s}, \nabla u_{+}^{i, s}\right)+f_{d_{i}}\left(x, u_{-}^{i, s}, \nabla u_{-}^{i, s}\right)\right] \mathrm{d} x \geq \\
\sum_{m=0}^{l_{i}-1} \int_{\left[0, s q_{1}\right] \times A_{\Gamma_{1}}}\left[f_{d_{i}}\left(x, u_{\max }^{i, m}+\phi_{i, m}, \nabla\left(u_{\max }^{i, m}+\phi_{i, m}\right)\right)+f_{d_{i}}\left(x, u_{\min }^{i, m}, \nabla u_{\min }^{i, m}\right)\right] \mathrm{d} x+a\left|A_{\Gamma_{i}}\right| .
\end{aligned}
$$


The last formula implies that there is at least one $u_{\max }^{i, \tilde{m}}+\phi_{i, \tilde{m}}$ or $u_{m i n}^{i, \tilde{m}}$, which we call $\tilde{u}$, such that

$$
\int_{\left[0, s q_{1}\right] \times A_{\Gamma_{i}}} f_{d_{i}}(x, \tilde{u}, \nabla \tilde{u}) \mathrm{d} x \leq \frac{1}{l_{i}} \int_{\left[0, s q_{1}\right] \times A_{\Gamma_{i}}}\left[f_{d_{i}}\left(x, u_{+}^{i, s}, \nabla u_{+}^{i, s}\right)+f_{d_{i}}\left(x, u_{-}^{i, s}, \nabla u_{-}^{i, s}\right)\right] \mathrm{d} x-\frac{1}{l_{i}} a\left|A_{\Gamma_{i}}\right| .
$$

By (2.46), we have

$$
\frac{1}{s} \int_{\left[0, s q_{1}\right] \times A_{\Gamma_{i}}} f_{d_{i}}(x, \tilde{u}, \nabla \tilde{u}) \mathrm{d} x \leq \frac{1}{s q_{1}\left|A_{\Gamma_{1}}\right|} \int_{\left[0, s q_{1}\right] \times A_{\Gamma_{i}}}\left[f_{d_{i}}\left(x, u_{+}^{i, s}, \nabla u_{+}^{i, s}\right)+f_{d_{i}}\left(x, u_{-}^{i, s}, \nabla u_{-}^{i, s}\right)\right] \mathrm{d} x-\frac{a}{s q_{1}} .
$$

We now denote by $T_{ \pm}^{i, s}$ the currents associated to $u_{ \pm}^{i, s}$ respectively; we denote by $A_{i}$ the action of $f_{d_{i}}$ and by $\alpha_{i}$ the $\alpha$-functional associated to $A_{i}$. As usual, we have that $\alpha_{i}(0)=0,-\alpha_{i}^{\prime}(0)=\rho_{i}$. In the first term of the last formula, we divided by $s q_{1}\left|A_{\Gamma_{i}}\right|$, the volume of the fundamental domain of $u_{ \pm}^{i, s}$; thus this term is the sum of the mean actions of $u_{+}^{i, s}$ and $u_{-}^{i, s}$, and we get

$$
\frac{1}{s} \int_{\left[0, s q_{1}\right] \times A_{\Gamma_{i}}} f_{d_{i}}(x, \tilde{u}, \nabla \tilde{u}) \mathrm{d} x \leq A_{i}\left(T_{+}^{i, s}\right)+A_{i}\left(T_{-}^{i, s}\right)-\frac{a}{s q_{1}} .
$$

On the other side, if $d_{ \pm}^{i, s}$ are such that $\alpha^{\prime}\left(d_{ \pm}^{i, s}\right)=\rho_{ \pm}^{i, s}$, we have that

$$
\begin{aligned}
0 & \geq \alpha_{i}\left(d_{-}^{i, s}\right)+\alpha_{i}\left(d_{+}^{i, s}\right)+\left\langle\rho^{i}, d_{+}^{i, s}+d_{-}^{i, s}\right\rangle=A_{i}\left(T_{-}^{i, s}\right)-\left\langle\rho_{-}^{i, s}, d_{-}^{i, s}\right\rangle+A_{i}\left(T_{+}^{i, s}\right)-\left\langle\rho_{+}^{i, s}, d_{+}^{i, s}\right\rangle+\left\langle\rho^{i}, d_{+}^{i, s}+d_{-}^{i, s}\right\rangle \\
& =A_{i}\left(T_{-}^{i, s}\right)+A_{i}\left(T_{+}^{i, s}\right)-\left\langle\frac{e_{1}}{s q_{1}}, d_{+}^{i, s}-d_{-}^{i, s}\right\rangle \\
& \geq \frac{1}{s} \int_{\left[0, s q_{1}\right] \times A_{\Gamma_{i}}}\left[f_{d_{i}}(x, \tilde{u}, \nabla \tilde{u})+f_{d_{i}}\left(x, u_{m i n}^{i, s}, \nabla u_{m i n}^{i, s}\right)\right] \mathrm{d} x+\frac{a}{s q_{1}}-\frac{\left\langle e_{1}, d_{+}^{i, s}-d_{-}^{i, s}\right\rangle}{s q_{1}} .
\end{aligned}
$$

In the formula above, the first inequality comes from the fact that $\alpha_{i}$ is concave, $\alpha_{i}(0)=0$ and $-\alpha_{i}^{\prime}(0)=\rho_{i}$; the first equality comes from the fact that $\alpha_{i}\left(d_{ \pm}^{i, s}\right)$ is realized by $T_{ \pm}^{i, s}$, the second equality from the definition of $\rho^{i}$ and $\rho_{ \pm}^{i, s}$; the last inequality comes from (2.53). Now the last formula implies that

$$
\int_{\left[0, s q_{1}\right] \times A_{\Gamma_{i}}} f_{d_{i}}(x, \tilde{u}, \nabla \tilde{u}) \mathrm{d} x<0
$$

if

$$
\left\langle e_{1}, d_{+}^{i, s}-d_{-}^{i, s}\right\rangle<a
$$

Since the integral above cannot be negative without contradicting the minimality of $u_{i}$, we get that

$$
\left|\left\langle e_{1}, d_{+}^{i, s}+d_{-}^{i, s}\right\rangle\right| \geq a
$$

which clearly implies the thesis. If $q_{1}$ is not relatively prime to the other denominators, the proof remains the same, only the arithmetic arguments become a little more complicated.

Lemma 2.13. Let us consider $D_{\rho}$, the flat of $\alpha$ of slope $\rho$; we have called $F_{D_{\rho}}$ the space it generates. Let $(c, \alpha(c)) \in D_{\rho}^{\circ}$. Then

$$
\operatorname{dim} F_{D_{\rho}}=\left\{\begin{array}{l}
0 \text { if } M_{\rho} \text { is an ordered set } \\
\operatorname{rat}(\rho, 1) \text { otherwise. }
\end{array}\right.
$$

Moreover, $\mathcal{A}_{c}$ is a $\rho$ lamination at $c$. 
Let $D_{\left(\rho, v_{1}\right)}$ be the face of $D_{\rho}$ with exterior normal $v_{1}$. Let $(c, \alpha(c)) \in D_{\left(\rho, v_{1}\right)}^{\circ}$; then

$$
\operatorname{dim} F_{D_{\left(\rho, v_{1}\right)}}=\left\{\begin{array}{l}
0 \text { if } M_{\left(\rho, v_{1}\right)} \text { is an ordered set } \\
\operatorname{rat}(\rho, 1) \cap \operatorname{rat} v_{1} \text { otherwise. }
\end{array}\right.
$$

Moreover, $\mathcal{A}_{c}$ is a $\left(\rho, v_{1}\right)$-lamination at $c$.

Similar statements hold for the faces of $D_{\left(\rho, v_{1}\right)}$, the faces of the faces, down to dimension zero.

Proof. Let us begin with the statement for $D_{\rho}$; since $(c, \alpha(c)) \in D_{\rho}^{\circ}$, we have that $\rho=-\alpha^{\prime}(c)$. We begin to take $d_{i} \equiv c$ in (2.4), and we get that $M_{-\alpha^{\prime}(c)}^{r e c}$ is contained in a $-\alpha^{\prime}(c)$-lamination at $c$; by Lemma 2.10,

$$
F_{D_{-\alpha^{\prime}(c)}} \subset \operatorname{rat}\left(-\alpha^{\prime}(c), 1\right)
$$

If $M_{\rho}$ is an ordered set, then by [4] there are only three possibilities: either $\operatorname{rat}\left(-\alpha^{\prime}(c), 1\right)$ is reduced to zero, or $M_{\rho}$ is a foliation, or both. In all these cases, $F_{D_{-\alpha^{\prime}(c)}}=\{0\}$ : in the first one by the last formula, in the second and third one by the last assertion of Lemma 2.10. This proves the first case of (2.54).

We prove the second case of (2.54). If $M_{-\alpha^{\prime}(c)}$ is not an ordered set, then we consider the lamination $\mathcal{C}$ of all the $u \in M_{-\alpha^{\prime}(c)}$ with $l(u)=0$. By [4], the gaps of $\mathcal{C}$ contain elements of $M_{-\alpha^{\prime}(c)}$ with different invariants, i.e. $\mathcal{C}$ satisfies the hypotheses of Lemma 2.12. By Lemma A3 of the appendix, we can suppose that

$$
-\alpha^{\prime}(c)=\left(\rho_{1}, \ldots, \rho_{l}, \rho_{l+1}, \ldots, \rho_{n}\right)
$$

with $\left(\rho_{1}, \ldots, \rho_{l}\right) \in \mathbf{Q}^{l}$ and $\left(\rho_{l+1}, \ldots, \rho_{n}\right)$ rationally independent. Let $\rho^{i} \in \mathbf{Q}^{n}$ be such that

$$
\rho^{i}=\left(\rho_{1}, \ldots, \rho_{l}, \rho_{l+1}^{i}, \ldots, \rho_{n}^{i}\right) \rightarrow\left(\rho_{1}, \ldots, \rho_{l}, \rho_{l+1}, \ldots, \rho_{n}\right)
$$

We can find $d_{i} \in H$ such that $-\alpha^{\prime}\left(d_{i}\right)=\rho^{i}$; since $-\alpha$ is superlinear and $-\alpha^{\prime}\left(d_{i}\right) \rightarrow-\alpha^{\prime}(c)$, we get that $\left\{d_{i}\right\}$

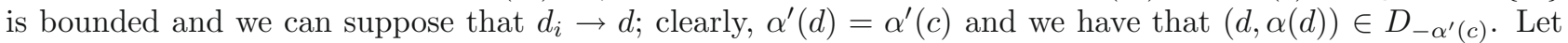
$u_{i} \in M_{\rho^{i}}^{r e c}$; since the rational coordinates of $\rho_{i}$ coincide with those of $-\alpha^{\prime}(c)$, one sees easily that $u_{i}$ converges to $u$ periodic in the first $l$ coordinates; this in turn implies that $l(u)=0$ and thus that $u \in \mathcal{C}$. Since the gaps of $\mathcal{C}$ contain elements with different invariants, we can apply Lemma 2.12 and get that the flat of $\alpha$ at $d_{i}$ contains a ball $B\left(\left(c_{i}, \alpha\left(c_{i}\right)\right), r\right) \cap \operatorname{rat}\left(\rho^{i}, 1\right)$, with $r$ which does not depend on $i$. We note that $c_{i}$ does not necessarily coincide with $d_{i}$, it only lies on the same flat. Denoting by $\tilde{d}$ the limit of the sequence $\left\{c_{i}\right\}$, we get that $\tilde{d}$ lies on the same flat as $c$ and $d$; moreover, this flat contains a ball $B((\tilde{d}, \alpha(\tilde{d})), r) \cap \operatorname{rat}\left(-\alpha^{\prime}(c), 1\right)$. Thus $F_{D_{-\alpha^{\prime}(c)}} \supset \operatorname{rat}\left(-\alpha^{\prime}(c), 1\right)$; since we have already proven the opposite inclusion, we have the second case of $(2.54)$.

We have to prove that $\mathcal{A}_{c}$ is a $\rho$-lamination at $c$. Let us consider the sequence $\left\{d_{i}\right\}$ of the paragraph above, and let $\mathcal{B}$ be the set of the limits of the elements of $M_{-\alpha^{\prime}\left(d_{i}\right)}$; the proof of Lemma 2.11 shows that $\mathcal{B}$ contains all the elements $u$ of $M_{-\alpha^{\prime}(c)}$ with $l(u)=0$, while from Lemma 2.3 we get that (2.3) and (2.4) hold. This proves that $\mathcal{A}_{c}$ contains a $-\alpha^{\prime}(c)$-lamination. Now $\mathcal{A}_{c}$ cannot contain any $\tilde{\rho}$-lamination with $\tilde{\rho} \neq-\alpha^{\prime}(c)$ : since $\alpha$ is $C^{1}$, it is easy to verify that no sequence $\left\{d_{i}\right\}$ can verify $(2.4)$ if $-\alpha^{\prime}\left(d_{i}\right)$ does not converge to $-\alpha^{\prime}(c)$. It remains to prove that $\mathcal{A}_{c}$ does not contain any $\left(-\alpha^{\prime}(c), v_{1}, \ldots, v_{s}\right)$-lamination at $c$ with $s \geq 1$; we shall prove this when $s=1$.

If $\operatorname{dim} F_{D_{-\alpha^{\prime}(c)}}>0, \mathcal{A}_{c}$ cannot contain any $\left(-\alpha^{\prime}(c), v_{1}\right)$-lamination, otherwise by Lemma $2.10 F_{D_{-\alpha^{\prime}(c)}} \subset$ $\operatorname{rat}\left(-\alpha^{\prime}(c), 1\right) \cap \operatorname{rat} v_{1}$, i.e. the dimension of $F_{D_{-\alpha^{\prime}(c)}}$ would be strictly smaller than the one given by (2.54). If $\operatorname{dim} F_{D_{-\alpha^{\prime}(c)}}=0$, there are two cases: in the first one, $\operatorname{rat}\left(-\alpha^{\prime}(c), 1\right)=\{0\}$; since by [4] $v_{1} \in \operatorname{rat}\left(-\alpha^{\prime}(c), 1\right)$, we cannot have any $\left(-\alpha^{\prime}(c), v_{1}\right)$-lamination at $c$. In the second one, $\operatorname{rat}\left(-\alpha^{\prime}(c), 1\right) \neq 0$, but $M_{-\alpha^{\prime}(c)}$ is an ordered set; by [4], these two conditions imply that $M_{-\alpha^{\prime}(c)}$ is a foliation. But then, again by [4], there is no $u \in M_{-\alpha^{\prime}}(c)$ with $l(u)>0$, and again the assertion follows. 
We now prove formula (2.55). Let us consider the face $D_{\left(\rho, v_{1}\right)}$ and let us suppose that $(c, \alpha(c)) \in D_{\left(\rho, v_{1}\right)}^{\circ}$. Again by Lemma A3 of the Appendix, we can suppose that $v_{1}$, the first invariant of $\mathcal{C}$, satisfies

$$
\pi_{-1}\left(v_{1}\right)=\left(\lambda_{1}, \ldots, \lambda_{m} \lambda_{m+1}, \ldots, \lambda_{l}, 0, \ldots, 0\right)
$$

with $\left(\lambda_{1}, \ldots, \lambda_{m}\right) \in \mathbf{Q}^{m}$ and $\left(\lambda_{m+1}, \ldots, \lambda_{l}\right)$ rationally independent. We want to show that there is a $\left(-\alpha^{\prime}(c), v_{1}\right)$ lamination at $c$. To this purpose, we consider $v$, the normal in $\pi_{-1}(\operatorname{rat}(\rho, 1))$ to $\pi_{-1}\left(D_{\left(\rho, v_{1}\right)}\right)$, and we let

$$
\rho_{i}=-\alpha^{\prime}(c)+\frac{1}{i} v
$$

We find $d_{i}$ be such that $-\alpha^{\prime}\left(d_{i}\right)=\rho_{i}$. We can suppose as before that $d_{i} \rightarrow d$, with $(d, \alpha(d)) \in D_{-\alpha^{\prime}(c)}$. Since $\alpha$ is concave, we have that, if $\alpha^{\prime}\left(c^{\prime}\right)=\alpha^{\prime}(c)$, then

$$
0 \geq\left\langle\alpha^{\prime}\left(d_{i}\right)-\alpha^{\prime}\left(c^{\prime}\right), d_{i}-c^{\prime}\right\rangle=\left\langle-\frac{1}{i} v, d_{i}-c^{\prime}\right\rangle .
$$

Passing to the limit, the inequality above implies that

$$
\left\langle v, d-c^{\prime}\right\rangle \geq 0
$$

if $c^{\prime} \in \pi_{-1}\left(D_{-\alpha^{\prime}(c)}\right)$; since equality is attained for $c^{\prime}=d$, we have that $d$ lies on the face of $\pi_{-1}\left(D_{-\alpha^{\prime}(c)}\right)$ with exterior normal $v$. Now we see that, by our choice of $\rho_{i}, u_{i} \in M_{\rho_{i}}^{r e c}$ converges to $u \in \mathcal{C}$; since $d_{i}$ satisfies condition $(2.6)$ of Lemma $2.3, \mathcal{C}$ is a $\left(-\alpha^{\prime}(c), v_{1}\right)$-lamination at $d$. We assert that $\mathcal{C}$ is a $\left(-\alpha^{\prime}(c), v_{1}\right)$-lamination also at $c$. This is because

$$
\begin{aligned}
\delta\left\|\pi_{0}\left(\alpha^{\prime}\left(d_{i}\right)-\alpha^{\prime}(d)\right)\right\| & \geq \alpha\left(d_{i}\right)+\left\langle\alpha^{\prime}\left(d_{i}\right), d-d_{i}\right\rangle-\alpha(d) \\
& =\alpha\left(d_{i}\right)+\left\langle\alpha^{\prime}\left(d_{i}\right), d-d_{i}\right\rangle-\left[\alpha(c)+\left\langle\alpha^{\prime}(c), d-c\right\rangle\right] \\
& =\alpha\left(d_{i}\right)+\left\langle\alpha^{\prime}\left(d_{i}\right), c-d_{i}\right\rangle-\alpha(c)
\end{aligned}
$$

where the first inequality is (2.4) at $d$, the first equality comes from the fact that $c$ and $d$ are on the same flat of $\alpha$ and the second one from (2.57) and the fact that $d_{i}-c \perp v$ since $d$ and $c$ are both on a face whose exterior normal is $v$. By the last formula, (2.4) holds at $c$ too, i.e. $\mathcal{C}$ is a $\left(-\alpha^{\prime}(c), v_{1}\right)$-lamination at $c$; since $(c, \alpha(c)) \in D_{\left(-\alpha^{\prime}(c), v_{1}\right)}^{\circ}$, we get by Lemma 2.10 that

$$
F_{D_{\left(-\alpha^{\prime}(c), v_{1}\right)}} \subset \operatorname{rat}\left(-\alpha^{\prime}(c), 1\right) \cap \operatorname{rat} v_{1}
$$

Now there are two cases: in the first one, $\mathcal{B}$, the $\left(-\alpha^{\prime}(c), v_{1}\right)$-lamination at $c$, is a foliation, or $\operatorname{rat}\left(-\alpha^{\prime}(c), v_{1}\right) \cap$ rat $v_{1}=0$; in this case, we prove as before that $F_{D_{\left(\rho, v_{1}\right)}}=0$. In the second case, the gaps of $\mathcal{B}$ contain elements with different invariants. By (2.56) and Lemma 2.12 the projections on $\mathbf{R}^{n}$ of the flats of $\alpha$ at $d_{i}$ contain balls in $\mathbf{R}^{m} \times\{0\}$ of fixed radius; thus the projection of the flat at $d$ contains a ball in $\mathbf{R}^{m} \times\{0\}$; but this means that

$$
\pi_{-1}\left(F_{D_{\left(-\alpha^{\prime}(c), v_{1}\right)}}\right) \supset \mathbf{R}^{m} \times\{0\}
$$

or, by $(2.56)$,

This proves the second case of $(2.55)$.

$$
F_{D_{\left(-\alpha^{\prime}(c), v_{1}\right)}} \supset \operatorname{rat}\left(-\alpha^{\prime}(c), 1\right) \cap \operatorname{rat} v_{1} .
$$

This also proves that there are no $\left(-\alpha^{\prime}(c), v_{1}, v_{2}\right)$-laminations at $c$ : if there were one, then by Lemma 2.10

$$
F_{D_{\left(-\alpha^{\prime}(c), v_{1}\right)}} \subset \operatorname{rat}\left(-\alpha^{\prime}(c), 1\right) \cap \operatorname{rat}\left(v_{1}\right) \cap \operatorname{rat}\left(v_{2}\right)
$$


contradicting (2.58). We have to prove that there are no $\left(-\alpha^{\prime}(c), v_{2}\right)$-laminations at $c$ with $v_{2} \neq \lambda v_{1}$ for any $\lambda>0$. We have just seen that, if at $c$ there were both a $\left(-\alpha^{\prime}(c), v_{1}\right)$ and a $\left(-\alpha^{\prime}(c), v_{2}\right)$-lamination, then $D_{-\alpha^{\prime}(c)}$ would have the exterior normals $v_{1}$ and $v_{2}$ and obviously all their linear combinations. Let $v_{1}$ and $v_{2}$ be as above; we assert that rat $v_{1}=\operatorname{rat} v_{2}$; indeed, if it were not so, then (2.59) would again contradict (2.58). Let us suppose by contradiction that

$$
\pi_{-1}\left(v_{2}\right)=\left(\lambda_{1}, \ldots, \lambda_{m}, \lambda_{m}^{\prime}, \ldots, \lambda_{l}^{\prime}, 0, \ldots, 0\right)
$$

with $\left(\lambda_{m+1}^{\prime}, \ldots, \lambda_{l}^{\prime}\right)$ rationally independent and $\left(\lambda_{m+1}^{\prime}, \ldots, \lambda_{l}^{\prime}\right) \neq\left(\lambda_{m+1}, \ldots, \lambda_{l}\right)$. Setting

$$
v_{\mu}=(1-\mu) v_{1}+\mu v_{2}
$$

we get that there is $\mu \in(0,1)$ such that $\operatorname{rat}\left(-\alpha^{\prime}(c), 1\right) \cap \operatorname{rat} v_{\mu}$ strictly contains $\operatorname{rat}\left(-\alpha^{\prime}(c), 1\right) \cap \operatorname{rat} v_{1}$. We also get as before that $\mathcal{A}_{c}$ contains a $\left(-\alpha^{\prime}(c), v_{\mu}\right)$-lamination $\mathcal{C}$. There are two cases: in the first one $\mathcal{C}$ satisfies the hypotheses of Lemma 2.12, and thus the dimension of $F_{D_{\left(-\alpha^{\prime}(c), v_{\mu}\right)}}=F_{D_{\left(-\alpha^{\prime}(c), v_{1}\right)}}$ is strictly larger than that of $\operatorname{rat}\left(-\alpha^{\prime}(c), 1\right) \cap \operatorname{rat} v_{1}$, a contradiction with Lemma 2.10. In the second one, $\mathcal{C}$ does not satisfy the hypotheses of Lemma 2.12, and then by Lemma $2.10 D_{\left(-\alpha^{\prime}(c), v_{\mu}\right)}$ is reduced to a point.

Now $\operatorname{rat}\left(-\alpha^{\prime}(c), 1\right) \cap \operatorname{rat} v_{\mu} \neq 0$, and the gaps of $\mathcal{C}$ do not contain elements with different invariants; this easily implies that $\mathcal{C}$ is a foliation. Let us consider the form $\eta_{(\mathcal{C}, c)}$ defined in $(2.10)$, and let

$$
\mathcal{L}_{1}(x, u, p)=\mathcal{L}(x, u, p)-\eta_{(\mathcal{C}, c)}^{x} \cdot p-\eta_{(\mathcal{C}, c)}^{u} \cdot
$$

The lamination of the $u \in M_{-\alpha^{\prime}(c)}$ with $l(u)=0$ has gaps, otherwise by [4] we would not have any $\left(-\alpha^{\prime}(c), v_{1}\right)$ lamination at $c$. Let $u_{1}<u_{2}$ be two elements of $M_{-\alpha^{\prime}(c)}$ with $l\left(u_{1}\right)=l\left(u_{2}\right)=0$; let us suppose that they bound a gap and let us consider the strip

$$
S_{\epsilon}=\left\{(x, y) \in \mathbf{R}^{n} \times \mathbf{R}: u_{1}(x)+\epsilon<y<u_{2}(x)-\epsilon\right\}
$$

Since $u_{1}<u_{2}$ the strip above is not empty if $\epsilon$ is sufficiently small. We recall that, if $u \in \mathcal{C}$, then $\nabla u(x)$ is the unique minimizer of $: p \rightarrow \mathcal{L}_{1}(x, u(x), p)$; by compactness, there is $a>0$ such that

$$
\mathcal{L}_{1}(x, w, \nabla w) \geq a
$$

if $w$ belongs to a $\left(-\alpha^{\prime}(c), v_{1}\right)$-lamination and $(x, w(x)) \in S_{\epsilon}$. Let now $\alpha^{\prime}\left(d_{i}\right) \in \mathbf{Q}^{n}$ and

$$
\alpha^{\prime}\left(d_{i}\right) \rightarrow \alpha^{\prime}(c) \quad \text { with } \quad \alpha^{\prime}\left(d_{i}\right)-\alpha^{\prime}(c) \in \pi_{-1}\left(\operatorname{rat}\left(-\alpha^{\prime}(c), 1\right)\right)
$$

If $\alpha^{\prime}\left(d_{i}\right)-\alpha^{\prime}(c)=\frac{1}{i} v_{1}$, we have that $u_{i} \in M_{-\alpha^{\prime}\left(d_{i}\right)}^{r e c}$ converge to the elements of a $\left(-\alpha^{\prime}(c), v_{1}\right)$-lamination. Moreover, it is easy to see that

$$
\lim _{R \rightarrow+\infty} \frac{1}{|B(0, R)|}\left|\left\{x \in B(0, R):(x, u(x)) \in S_{\epsilon}\right\}\right| \geq C\left\|\pi_{0}\left(\alpha^{\prime}\left(d_{i}\right)-\alpha^{\prime}(c)\right)\right\|
$$

where $\pi_{0}$ is the projection on $\pi_{-1}\left(\operatorname{rat}\left(-\alpha^{\prime}(c), 1\right)\right)$. By the last three formulas and the fact that $\mathcal{L}_{1}(x, u, p) \geq 0$, we get that, denoting by $T_{i}$ the current induced by $u_{i}$,

$$
A\left(T_{i}\right) \geq C a\left\|\alpha^{\prime}\left(d_{i}\right)-\alpha^{\prime}(c)\right\|
$$

a contradiction with Lemma 2.3.

Using induction and a similar argument, the lemma follows for all subfaces. 
Proof of proposition 2.4. The direct part of point 1) is Lemma 2.5. Points 2) and 3) follow from Lemma 2.13. The converse part of point 1) follows from point 2): if $\mathcal{A}_{c_{1}}$ is a $\left(\rho, v_{1}, \ldots, v_{s}\right)$-lamination and $\mathcal{A}_{c_{1}} \subset \mathcal{A}_{c_{2}}$, then by point 2$) \mathcal{A}_{c_{2}}$ is a $\left(\rho, v_{1}, \ldots, v_{s}, v_{s+1}, \ldots, v_{l}\right)$-lamination; again by point 2$), c_{1} \in D_{\left(\rho, v_{1}, \ldots, v_{s}\right)}^{\circ}$, while $c_{2} \in D_{\left(\rho, v_{1}, \ldots, v_{s} v_{s+1}, \ldots, v_{l}\right)}^{\circ}$, i.e. $c_{2}$ belongs to a subface of $c_{1}$.

\section{Appendix}

Lemma A1. Let $\rho \in \mathbf{Q}^{n}$, let $u \in M_{\rho}^{r e c}$ and let

$$
\Gamma=\left\{k \in \mathbf{Z}^{n}: \rho \cdot k \in \mathbf{Z}\right\}
$$

Let also $(\bar{k}, \bar{j})$ be such that

$$
u(x+\bar{k})+\bar{j}-u(x)=\min \left\{u(x+k)+j-u(x): u(x+k)+j-u(x)>0, \quad(k, j) \in \mathbf{Z}^{n} \times \mathbf{Z}\right\} .
$$

Then the following holds.

1) The set of the minima $(\bar{k}, \bar{j})$ does not depend on $x$; moreover, $(\bar{k}, \bar{j})$ satisfies the equation above iff

$$
\rho \cdot \bar{k}+\bar{j}=\min \left\{\rho \cdot k+j: \rho \cdot k+j>0, \quad(k, j) \in \mathbf{Z}^{n} \times \mathbf{Z}\right\} .
$$

2) If $A_{\Gamma}$ is a fundamental domain for $\Gamma$, then the set

$$
S=\left\{\left(x, x_{n+1}\right): x \in A_{\Gamma}, \quad u(x) \leq x_{n+1}<u(x+\bar{k})+\bar{j}\right\}
$$

projects injectively and surjectively onto $\mathbf{T}^{n+1}$.

3) The Lebesgue measure of $S$ is 1 .

4) $l=\frac{1}{\rho \cdot k+\bar{j}} \in \mathbf{N}$ and $u(x+l \bar{k})+l \bar{j}=u(x)+1$.

Proof. Point 1) follows easily from a fact proven in [17]: the set $\left\{u(x+k)+j:(k, j) \in \mathbf{Z}^{n} \times \mathbf{Z}\right\}$ is ordered, and $u(x+k)+j>u(x)$ iff $\rho \cdot k+j>0$.

As for point 2), we begin to prove that the projection of $S$ on $\mathbf{T}^{n+1}$ is injective. Let us suppose by contradiction that $\left(x, x_{n+1}\right) \in S$ and $\left(x+\tilde{k}, x_{n+1}+\tilde{j}\right) \in S$ for some $(\tilde{k}, \tilde{j}) \in\left(\mathbf{Z}^{n} \times \mathbf{Z}\right) \backslash\{0\}$. Since $\left(x, x_{n+1}\right) \in S$ we have that

$$
x_{n+1}<u(x+\bar{k})+\bar{j} \quad \text { and } \quad u(x) \leq x_{n+1}
$$

and since $\left(x+\tilde{k}, x_{n+1}+\tilde{j}\right) \in S$ we have that

$$
u(x+\tilde{k}) \leq x_{n+1}+\tilde{j} \quad \text { and } \quad x_{n+1}+\tilde{j}<u(x+\tilde{k}+\bar{k})+\bar{j} .
$$

From the left sides of the two formulas above we get that

$$
u(x+\bar{k})+\bar{j}>u(x+\tilde{k})-\tilde{j}
$$

which contradicts the minimality of $(\bar{k}, \bar{j})$ when $u(x+\tilde{k})-\tilde{j}>u(x)$. The case $u(x+\tilde{k})-\tilde{j} \equiv u(x)$ cannot happen, because by the periodicity of $u$ it would imply that $\tilde{k} \in \Gamma$; but this contradicts the fact that $x$ and $x+\tilde{k}$ belong to $A_{\Gamma}$, the fundamental domain of $\Gamma$. It remains the case $u(x+\tilde{k})-\tilde{j}<u(x)$; in this case, we use the right sides of the two formulas above and we get that

$$
u(x+\tilde{k}+\bar{k})+\bar{j}>u(x)+\tilde{j}
$$

or equivalently

$$
u(y+\bar{k})+\bar{j}>u(y-\tilde{k})+\tilde{j} .
$$


Since $u(y-\tilde{k})+\tilde{j}>u(y)$, we again get that $(\bar{k}, \bar{j})$ is not minimal. This proves that $S$ projects injectively.

Before proving that $S$ projects surjectively, we prove point 4$)$. It is easy to see that $\rho \cdot \bar{k}+\bar{j} \in(0,1)$ : it is greater than zero by definition, and if it were larger than 1 , we could show that $(\bar{k}, \bar{j})$ is not minimal simply considering $(\bar{k}, \bar{j}-1)$. Let $l \in \mathbf{N}$ be such that $l(\rho \cdot \bar{k}+\bar{j}) \leq 1$ is maximal; in particular,

$$
0 \leq 1-l(\rho \cdot \bar{k}+\bar{j})<l(\rho \cdot \bar{k}+\bar{j})
$$

Now, $1-l(\rho \cdot \bar{k}+\bar{j})=0$, otherwise we would have two strict inequalities in the formula above, and this would contradict the minimality of $(\bar{k}, \bar{j})$. Thus we get

$$
l(\rho \cdot \bar{k}+\bar{j})=1
$$

which is point 4).

We now show that $S$ projects surjectively onto $\mathbf{T}^{n+1}$. Let $\left(x, x_{n+1}\right)$ be such that

$$
u(x) \leq x_{n+1}<u(x)+1 .
$$

We have to prove that an integer translate of $\left(x, x_{n+1}\right)$ belongs to $S$. Since $u$ is non self intersecting, we have that

$$
u(x)<u(x+\bar{k})+\bar{j}<u(x+2 \bar{k})+2 \bar{j}<\ldots<u(x+l \bar{k})+l \bar{j}=u(x)+1
$$

where the last equality comes from point 4$)$. Thus

$$
u(x+s \bar{k})+s \bar{j} \leq x_{n+1}<u(x+(s+1) \bar{k})+(s+1) \bar{j}
$$

for some $0 \leq s \leq l-1$. But this means that $\left(x+s \bar{k}, x_{n+1}-s \bar{j}\right) \in S$, which yields surjectivity.

Now point 3) follows immediately from point 2 ), because $S$ is brought bijectively onto $\mathbf{T}^{n+1}$ by the projection, a measure-preserving map.

Lemma A2. Let

$$
\left(\frac{p_{1}}{q_{1}}, \ldots, \frac{p_{n-1}}{q_{n-1}}, \frac{p_{n}}{q_{n}}\right) \in \mathbf{Q}^{n}
$$

and let $q_{n}$ be relatively prime to $q_{i}$ for $1 \leq i \leq n-1$. Let $A_{\Gamma^{\prime}}$ be a fundamental domain for

$$
\Gamma^{\prime}=\left\{k^{\prime} \in \mathbf{Z}^{n-1}: k_{1}^{\prime} \frac{p_{1}}{q_{1}}+\ldots+k_{n-1}^{\prime} \frac{p_{n-1}}{q_{n-1}} \in \mathbf{Z}\right\} .
$$

Then $A_{\Gamma^{\prime}} \times\left[0, q_{n}\right)$ is a fundamental domain for

$$
\Gamma=\left\{k \in \mathbf{Z}^{n}: k_{1} \frac{p_{1}}{q_{1}}+\ldots+k_{n} \frac{p_{n}}{q_{n}} \in \mathbf{Z}\right\} .
$$

Proof. It is easy to see that, if the statement is true for a particular fundamental domain $A_{\Gamma^{\prime}}$ of $\Gamma^{\prime}$, then it is true for any fundamental domain. We choose a particular $A_{\Gamma^{\prime}}$ in the following way: we pick $k_{1} \in \Gamma^{\prime} \backslash\{0\}$ of minimal norm, $k_{2} \in \Gamma^{\prime} \backslash \mathbf{R} k_{1}$ of minimal distance from $\mathbf{R} k_{1}$, etc. It is a standard fact that $k_{1}, \ldots, k_{n-1}$ are a basis of $\Gamma^{\prime}$, and that

$$
A_{\Gamma^{\prime}}=\left\{x^{\prime} \in \mathbf{R}^{n-1}: x^{\prime}=t_{1} k_{1}+\ldots+t_{n-1} k_{n-1}, \quad 0 \leq t_{i}<1\right\}
$$

is a fundamental domain of $\Gamma^{\prime}$. We can always build a fundamental domain from a base using the formula above; thus, to prove that $A_{\Gamma^{\prime}} \times\left[0, q_{n}\right)$ is a fundamental domain of $G$, is equivalent to prove that $k_{1}, \ldots, k_{n-1}, q_{n}$ 
is a base of $\Gamma$. Let us suppose by contradiction that it is not: thus we can find $\left(\alpha_{1}, \ldots, \alpha_{n}\right) \in \Gamma$ which is not generated by the vectors $\left(k_{1}, \ldots, k_{n-1}, q_{n}\right)$. Since $\left(\alpha_{1}, \ldots, \alpha_{n}\right) \in \Gamma$, we have that

$$
\frac{p_{1}}{q_{1}} \alpha_{1}+\ldots+\frac{p_{n-1}}{q_{n-1}} \alpha_{n-1}+\frac{p_{n}}{q_{n}} \alpha_{n} \in \mathbf{Z}
$$

There are two cases. If $\left(\alpha_{1}, \ldots, \alpha_{n-1}\right)$ is not generated by the basis of $\Gamma^{\prime}, k_{1}, \ldots, k_{n-1}$, then $\frac{p_{1}}{q_{1}} \alpha_{1}+\ldots+\frac{p_{n-1}}{q_{n-1}} \alpha_{n-1}$ is not an integer, but a fraction containing some factor of $q_{i}$ in the denominator; since $q_{n}$ is relatively prime to $q_{i}$, it is easy to see that the sum displayed above is not an integer. On the other side, if $\left(\alpha_{1}, \ldots, \alpha_{n-1}\right)$ is generated by the vectors $k_{1}, \ldots, k_{n-1}$, but $\alpha_{n}$ is not a multiple of $q_{n}$, then the sum of the first $n-1$ terms in the formula above is an integer, but $\frac{p_{n}}{q_{n}} \alpha_{n} \notin \mathbf{Z}$, and again the full sum is not in $\mathbf{Z}$.

The following well-known lemma simplifies many calculations; we recall that a vector $v \in \mathbf{R}^{s}$ is rationally independent if there is no $k \in \mathbf{Z}^{s} \backslash\{0\}$ such that $k \cdot v \in \mathbf{Z}$.

Lemma A3. Let $u \in M_{\rho}$ have invariants $\rho, v_{1}, \ldots, v_{l}$. Then, up to a unimodular transformation of $\mathbf{R}^{n}$, we can suppose that

$$
\rho=\left(\rho_{1}, \ldots, \rho_{s}, \rho_{s+1}, \ldots, \rho_{n}\right)
$$

with $\left(\rho_{1}, \ldots, \rho_{s}\right) \in \mathbf{Q}^{s}$ and $\left(\rho_{s+1}, \ldots, \rho_{n}\right)$ rationally independent. Moreover, if we set $v_{i}=\left(v_{i}^{x}, v_{i}^{u}\right)$ as in Section 1, we can suppose that

$$
v_{1}^{x}=\left(v_{1}^{1}, \ldots, v_{1}^{s^{\prime}}, v_{1}^{s^{\prime}+1}, \ldots, v_{1}^{s}, 0, \ldots, 0\right), \quad v_{1}=\left(v_{1}^{x},-\rho \cdot v_{1}^{x}\right)
$$

with $\left(v_{1}^{1}, \ldots, v_{1}^{s^{\prime}}\right) \in \mathbf{Q}^{s^{\prime}}$ and $\left(v_{1}^{s^{\prime}+1}, \ldots, v_{1}^{s}\right)$ rationally independent, etc.

Proof. We must show that there is $A$, a unimodular transformation of $\mathbf{R}^{n}$ which brings the rotation vector $\rho$ to the required form. We consider

$$
\Gamma=\left\{k \in \mathbf{Z}^{n}: \rho \cdot k \in \mathbf{Z}\right\} .
$$

If $\Gamma$ is $n$-dimensional, we have that $\rho \in \mathbf{Q}^{n}$ and taking the identity matrix will do. If the dimension of $\Gamma$ is $s<n$, we build a basis of $\mathbf{R}^{n}$ in the following way.

Let us begin to suppose that $\Gamma$ has the following property: for $k \in \Gamma$, all the integer vectors on the ray from 0 to $k$ are in $\Gamma$.

We take $k_{1} \in \Gamma \backslash\{0\}$ of minimal norm, $k_{2} \in \Gamma \backslash\left\{\mathbf{R} k_{1}\right\}$ of minimal distance from $\left\{\mathbf{R} k_{1}\right\}$, up to $k_{s} \in \Gamma$. We take $k_{s+1} \in \mathbf{Z}^{n} \backslash \Gamma$ of minimal distance from $\Gamma, k_{s+2} \in \mathbf{Z}^{n} \backslash<\Gamma, k_{s+1}>$ of minimal distance from $<\Gamma, k_{s+1}>$, etc. We define

$$
B_{\Gamma}=\left\{t_{1} k_{1}+\ldots+t_{n} k_{n}, 0 \leq t_{1}<1\right\}
$$

It is clear that translating $B_{\Gamma}$ by the elements of $\mathbf{Z}^{n}$ we cover all $\mathbf{R}^{n}$, and this implies that $\left|B_{\Gamma}\right| \geq 1$. To show that $\left|B_{\Gamma}\right| \leq 1$, by Theorem 3.34 of [11] it suffices to show that $B_{\Gamma}$ does not contain elements of $\mathbf{Z}^{n}$ in its interior; but this is an easy consequence of the choice of the $k_{i}$ and of our hypothesis on the rays of $\Gamma$. Now the matrix $A=\left(k_{1}, \ldots, k_{n}\right)$ brings the unit cube into $B_{\Gamma}$; since $\left|B_{\Gamma}\right|=1,\left(k_{1}, \ldots, k_{n}\right)$ is unimodular; we assert that $A^{T}$ is the matrix we are looking for. Indeed, $A$ brings the unit cube into $B_{\Gamma}$, and thus it brings $\mathbf{Z}^{s} \times\{0\}$ into $\Gamma$; now $\left\langle\rho A^{T}, k\right\rangle$ is an integer iff $\langle\rho, A k\rangle$ is an integer, and we know that this happens iff $A k \in \Gamma$, i.e. iff $k \in \mathbf{Z}^{s} \times\{0\}$. To state it differently,

$$
\left(\rho A^{T}, 1\right)^{\perp} \cap\left(\mathbf{Z}^{n} \times \mathbf{Z}\right)=\left\{\left(k,-\left\langle\rho A^{T}, k\right\rangle\right): k \in \mathbf{Z}^{s} \times\{0\}\right\} .
$$

But this means that $\left(\rho A^{T}, 1\right)^{\perp}$ contains $\left(e_{1}, j_{1}\right), \ldots,\left(e_{s}, j_{s}\right)$ with $j_{1}, \ldots, j_{s} \in \mathbf{Z}$. In other words, $\left\langle\rho A^{T}, e_{i}\right\rangle+j_{i}=0$ if $i \in(1, \ldots, s)$, i.e. the first $s$ coordinates of $\rho A^{T}$, the slope of $u \circ A^{T}$, are integers.

Let us now suppose that $\Gamma$ does not have the property mentioned above; we extend $\Gamma$ to $\tilde{\Gamma}$ adding all the integer vectors on the rays $\overline{0 k}$ with $k \in \Gamma$. Starting from $\tilde{\Gamma}$, we build a basis $k_{1}, \ldots, k_{n}$ as we did before for $\Gamma$. 
Since $k_{i} \in \tilde{\Gamma}$, if $k_{i} \notin \Gamma$ we can find a minimal $r_{i} \in \mathbf{N}$ such that $r_{i} k_{i} \in \Gamma$. We consider the matrix $A=\left(k_{1}, \ldots, k_{n}\right)$ and we see as before that it is unimodular; moreover, it brings the module generated by $r_{1} e_{1}, \ldots, r_{s} e_{s}$ into $\Gamma$; the same argument we used above shows that $\left\langle\rho A^{T}, r_{i} e_{i}\right\rangle+j_{i}=0$ for $i \in(1, \ldots, s)$, i.e. the first $s$ coordinates of $\rho A^{T} \in \mathbf{Q}^{s} \times\{0\}$ are rational.

It remains to prove that $\left(\rho_{s+1}, \ldots, \rho_{n}\right)$ is rationally independent, but if it were not so, then we could find $\bar{k} \in\{0\} \times \mathbf{Z}^{n-s}$ such that $A \bar{k} \cdot\left(\rho_{s+1}, \ldots, \rho_{n}\right) \in \mathbf{Z}$. But then the module of integer vectors orthogonal to $\left(\rho A^{T}, 1\right)$ would generate

$$
\left\{\left(k,-\left\langle\rho A^{T}, k\right\rangle\right): k \in \mathbf{Z}^{s} \times\{0\}\right\}+\langle(A \bar{k},-\rho \cdot A \bar{k})\rangle
$$

contradicting the fact that $\left(\rho A^{T}, 1\right)^{\perp} \cap\left(\mathbf{Z}^{n} \times \mathbf{Z}\right)$ projects to $\mathbf{Z}^{s}$.

We now show that we can bring $v_{1}$ to the required form by another unimodular transformation; we shall suppose that $\rho=\left(\rho_{1}, \ldots, \rho_{s}, \rho_{s+1}, \ldots, \rho_{n}\right)$, with $\left(\rho_{1}, \ldots, \rho_{s}\right) \in \mathbf{Q}^{n}$ and $\left(\rho_{s+1}, \ldots, \rho_{n}\right)$ rationally independent. Since $v_{1}$ is admissible, we have that $v_{1} \in \operatorname{rat}(\rho, 1)$, i.e. $v_{1}=\left(\alpha_{1}, \ldots, \alpha_{s}, 0, \ldots, 0, \alpha_{n+1}\right)$ with $\alpha_{n+1}=$ $-\left\langle\rho,\left(\alpha_{1}, \ldots, \alpha_{s}, 0, \ldots, 0\right)\right\rangle$. The projection of rat $v_{1}$ on $\mathbf{R}^{s}$ coincides with a certain module $\Gamma \subset \mathbf{Z}^{s}$. But we have just seen how to find a unimodular transformation of $\mathbf{R}^{s}$ bringing the first $s$ coordinates of $v_{1}$ to the form $\left(\alpha_{1}, \ldots, \alpha_{s^{\prime}}, \alpha_{s^{\prime}+1}, \ldots, \alpha_{s}, 0, \ldots, 0\right)$ with $\left(\alpha_{1}, \ldots, \alpha_{s^{\prime}}\right) \in \mathbf{Q}^{s^{\prime}}$ and $\left(\alpha_{s^{\prime}+1}, \ldots, \alpha_{s}\right)$ rationally independent.

\section{REFERENCES}

[1] G. Alberti, L. Ambrosio and X. Cabré, On a long standing conjecture of De Giorgi: symmetry in 3d for general nonlinearities and a local minimality property. Acta Appl. Math. 65 (2001) 9-33.

[2] S. Aubry and P.Y. Le Daeron, The discrete Frenkel-Kontorova model and its extensions. Physica 8D (1983) $381-422$.

[3] F. Auer and V. Bangert, Differentiability of the stable norm in codimension one. CRAS 333 (2001) 1095-1100.

[4] V. Bangert, On minimal laminations of the torus. Ann. Inst. H. Poincaré Anal. Non Linéaire 6 (1989) 95-138.

[5] V. Bangert, Geodesic rays, Busemann functions and monotone twist maps. Calc. Var. 2 (1994) 49-63.

[6] P. Bernard and B. Buffoni, Optimal mass transportation and Mather theory. J. Eur. Math. Soc. 9 (2007) 85-121.

[7] D. Burago, S. Ivanov and B. Kleiner, On the structure of the stable norm of periodic metrics. Math. Res. Lett. 4 (1997) 791-808.

[8] L. De Pascale, M.S. Gelli and L. Granieri, Minimal measures, one-dimensional currents and the Monge-Kantorovich probem. Calc. Var. Partial Differential Equations 27 (2006) 1-23.

[9] K. Deimling, Nonlinear Functional Analysis. Springer, Berlin (1985).

[10] M.P. do Carmo, Differential Forms and Applications. Springer, Berlin (1994).

[11] G.H. Hardy and E.M. Wright, An Introduction to the Theory of Numbers. Oxford (1980).

[12] D. Massart, Stable norms of surfaces: local structure of the unit ball at rational directions. GAFA 7 (1997) 996-1010.

[13] D. Massart, On Aubry sets and Mather's action functional. Israel J. Math. 134 (2003) 157-171.

[14] J.N. Mather, Differentiability of the minimal average action as a function of the rotation number. Bol. Soc. Bras. Mat. 21 (1990) 59-70.

[15] J.N. Mather, Action minimizing invariant measures for positive-definite Lagrangian systems. Math. Zeit. 207 (1991) $169-207$.

[16] J.N. Mather, Variational construction of connecting orbits. Ann. Inst. Fourier 43 (1993) 1349-1386.

[17] J. Moser, Minimal solutions of variational problems on a torus. Ann. Inst. H. Poincaré Anal. Non Linéaire 3 (1989) $229-272$.

[18] O. Osuna, Vertices of Mather's beta function. Ergodic Theory Dynam. Systems 25 (2005) 949-955.

[19] P.H. Rabinowitz and E. Stredulinsky, Mixed states for an Allen-Cahn type equation. Comm. Pure Appl. Math. 56 (2003) 1078-1134.

[20] W. Senn, Strikte Konvexität für Variationsprobleme auf dem n-dimensionalen Torus. Manuscripta Math. 71 (1991) 45-65.

[21] W. Senn, Differentiability properties of the minimal average action. Calc. Var. Partial Differential Equations 3 (1995) 343-384.

[22] W. Senn, Equilibrium form of crystals and the stable norm. Z. angew. Math. Phys. 49 (1998) 919-933.

[23] J.E. Taylor, Crystalline variational problems. BAMS 84 (1978) 568-588.

[24] M.E. Taylor, Partial Differential Equations, Basic Theory. Springer, Berlin (1996).

[25] N. Wiener, The ergodic theorem. Duke Math. J. 5 (1939) 1-18. 